\title{
Dearomatization Through Photoredox Hydroarylation: Discovery of Radical-Polar Crossover Strategy
}

\author{
Kelly A. McDaniel, Nathan T. Jui* \\ njui@emory.edu
}

Department of Chemistry and Winship Cancer Institute, Emory University, Atlanta, GA 30322

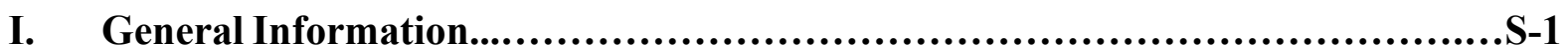

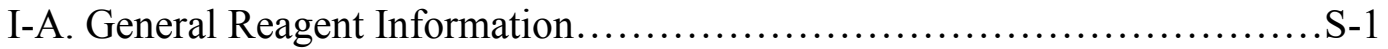

I-B. General Analytical Information.......................................

I-C. General Photoredox Reaction Setup................................. 1

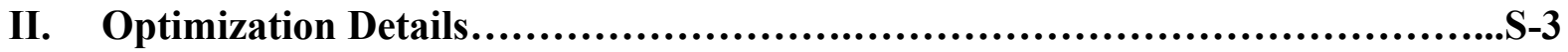

II-A. Optimization Procedure A..................................................

II-B. Optimization Procedure B ............................................

II-C. Gas Chromatography Method Conditions ..................................S-3

II-D. Optimization Table A..................................................

II-E. Optimization Table B...............................................

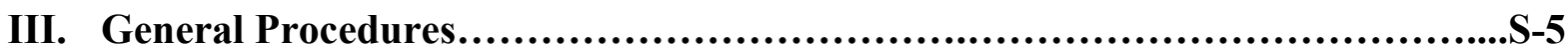

III-A. Acylation Procedure A.................................................. 5

III-B. Acylation Procedure B................................................ -5

III-C. General Hydroarylation Procedure A.................................. -5

III-D. General Hydroarylation Procedure B..............................

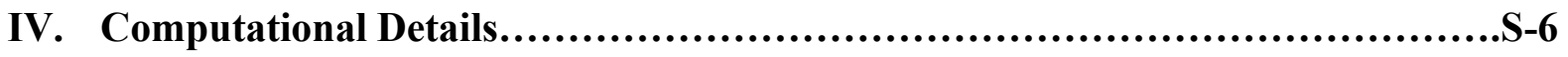

V. Preparation of Starting Materials.................................................9

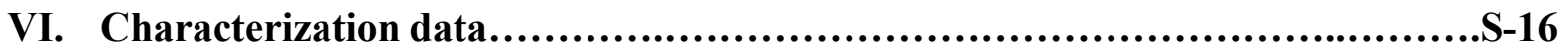

VII. Deuterium Labeling Study ......................................................23

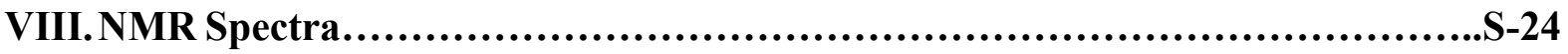




\section{General}

\section{I-A. General Reagent Information:}

Reagents were purchased from Sigma-Aldrich, Alfa Aesar, Acros Organics, Combi-Blocks, Oakwood Chemicals, Astatech, and TCI America and used as received, unless stated otherwise.

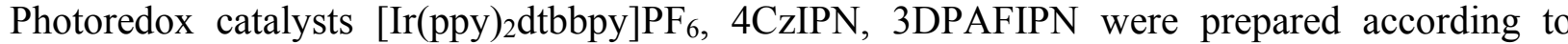
literature procedure. ${ }^{1,2}$ Organic solutions were concentrated under reduced pressure on a rotary evaporator using a water bath. Chromatographic purification of products was accomplished using forced-flow chromatography on 230-400 mesh silica gel. Thin-layer chromatography (TLC) was performed on $250 \mu \mathrm{m}$ SiliCycle silica gel F-254 plates. Visualization of the developed plates was performed by fluorescence quenching or staining using $\mathrm{KMnO}_{4}$. All reactions were conducted under nitrogen atmosphere while subjected to irradiation from blue LEDs (HydroFarm powerPAR LED Grow Light Blue $15 \mathrm{Watt} / 440 \mathrm{~nm}$, available at www.1000bulbs.com). Reaction solvent was prepared by degassing under weak vacuum while subjected to sonication.

\section{I-B. General Analytical Information:}

Unless otherwise noted, all yields refer to chromatographically and spectroscopically ( ${ }^{1} \mathrm{H}$ NMR) homogenous materials. New compounds were characterized by NMR, IR, and HRMS. ${ }^{1} \mathrm{H}$ and ${ }^{13} \mathrm{C}$ NMR spectra were obtained from the Emory University NMR facility and recorded on an INOVA 600 (600 MHz), INOVA 500 (500 MHz), INOVA 400 (400 MHz), or VNMR 400 (400 MHz), and are internally referenced to residual protio solvent signals. Data for ${ }^{1} \mathrm{H}$ NMR are reported as follows: chemical shift ( $\mathrm{ppm})$, multiplicity $(\mathrm{s}=$ singlet, $\mathrm{d}=$ doublet, $\mathrm{t}=$ triplet, $\mathrm{q}=$ quartet, $\mathrm{m}=$ multiplet, $\mathrm{dd}=$ doublet of doublets, $\mathrm{dt}=$ doublet of triplets, $\mathrm{ddd}=$ doublet of doublet of doublets, $\mathrm{dtd}=$ doublet of triplet of doublets, $\mathrm{b}=$ broad, etc.), coupling constant $(\mathrm{Hz})$, and integration. Data for decoupled ${ }^{13} \mathrm{C}$ NMR are reported in terms of chemical shift and multiplicity when applicable. IR spectra were recorded on a Thermo Fisher DiamondATR and reported in terms of frequency of absorption $\left(\mathrm{cm}^{-1}\right)$. High Resolution mass spectra were obtained from the Emory University Mass Spectral facility.

\section{I-C. General Photoredox Reaction Setup:}

To run multiple reactions (for either optimization or product preparation) an appropriately sized 3D printed carousel was used, which exposed the reactions to the blue light evenly (photo 1). A 15 W LED array lamp was used as a blue light source (photo 1,2). These lamps were routinely used for up to 12 reactions at a time. The reactions were cooled with a line of compressed air (photo 1). The blue LEDs were positioned approximately $6 \mathrm{~cm}$ above the reaction vials to get good light coverage without overheating the reactions (photo 1,2). Reactions run at elevated temperatures were irradiated in a shallow oil bath (photo 3,4 )

\footnotetext{
${ }^{1}$ Slinker, J. D.; Gorodetsky, A. A.; Lowry, M. S.; Wang, J.; Parker, S.; Rohl, R.; Bernhard, S.; Malliaras, G. G. Efficient Yellow Electroluminescence from a Single Layer of a Cyclometalated Iridium Complex. J. Am. Chem. Soc. 2004, 126, 2763-2767.

${ }^{2}$ Speckmeier, E.; Fischer, T. G.; Zeitler, K. A Toolbox Approach To Construct Broadly Applicable Metal-Free Catalysts for Photoredox Chemistry: Deliberate Tuning of Redox Potentials and Importance of Halogens in DonorAcceptor Cyanoarenes. J. Am. Chem. Soc. 2018, 140, 15353-15365.
} 


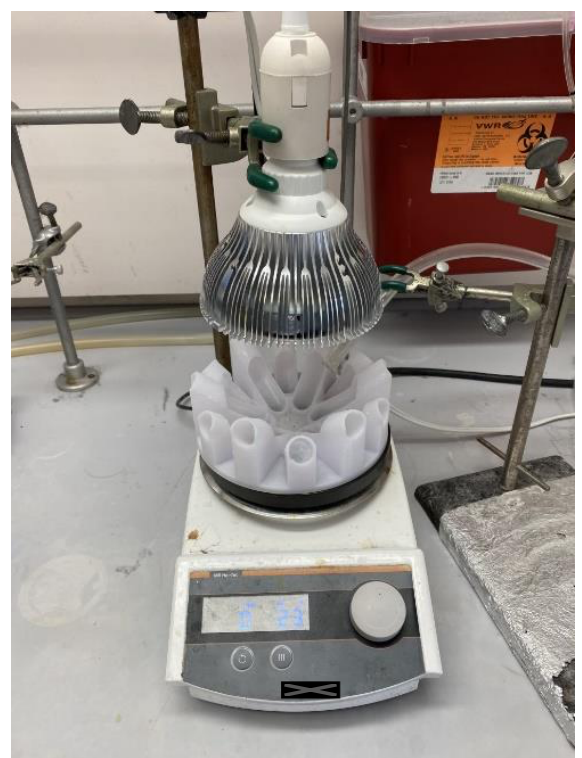

Photo 1

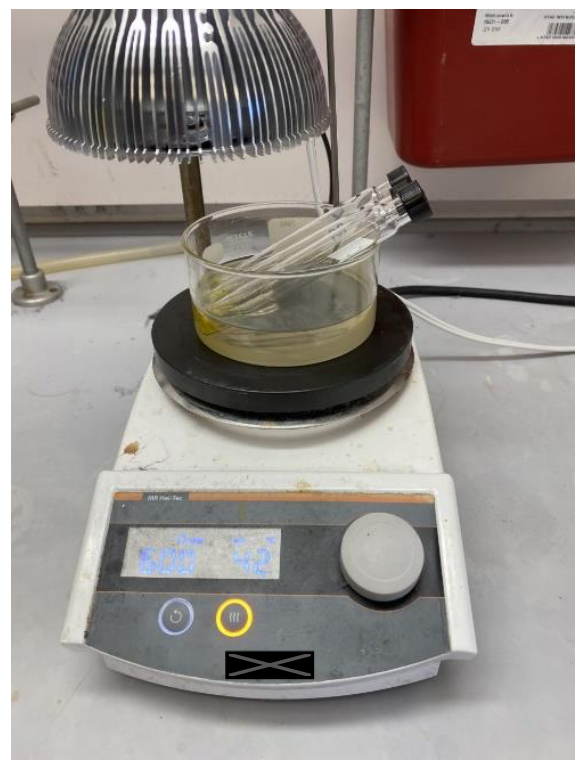

Photo 3

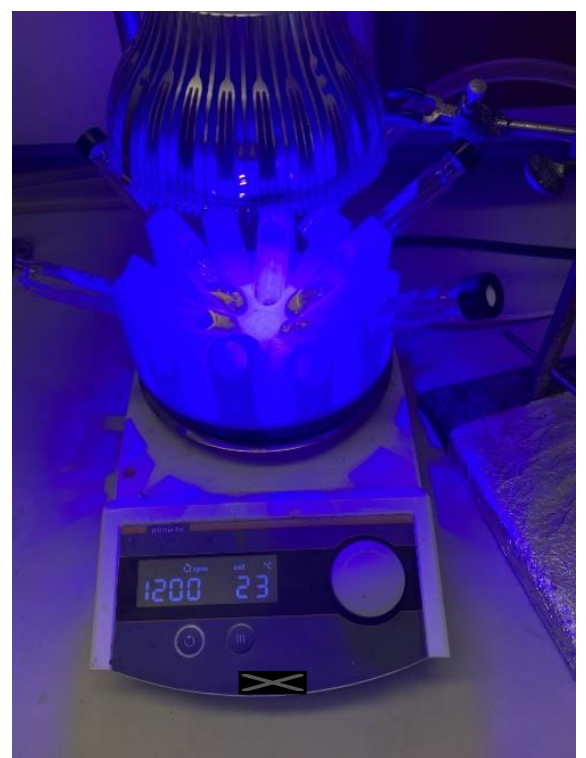

Photo 2

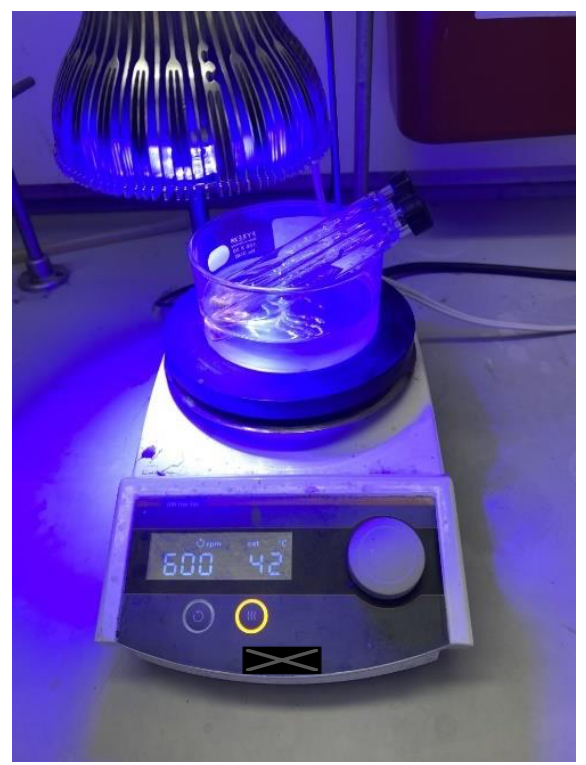

Photo 4 


\section{Optimization Details}

\section{II-A: Optimization Procedure A (Optimization Table A)}

A screw-top test tube equipped with a stir bar was charged with the photocatalyst (1 mol\%), (2bromopyridin-3-yl)(1H-indol-1-yl)methanone (1) (30.1 mg, $0.1 \mathrm{mmol}, 1$ equiv), and Hantzsch ester and sealed with a PTFE/silicon septum. The atmosphere was exchanged three times by applying vacuum and backfilling with $\mathrm{N}_{2}$. Under $\mathrm{N}_{2}$ atmosphere, thiophenol (5 mol\%) and acetic acid were added via microsyringe. Degassed 2,2,2-trifluoroethanol was added via syringe and the resulting mixture was stirred for 16 hours under irradiation with blue LEDs. The reaction was quenched with saturated aqueous sodium bicarbonate and extracted three times with ethyl acetate. The combined organic extracts were filtered through a silica plug which was washed with ethyl acetate. An internal standard of dodecane $(10 \mu \mathrm{L}, 0.044 \mathrm{mmol})$ was added. A sample was analyzed by GC-FID, and the integral values were used to calculate the data given in Table 1.

\section{II-B: Optimization Procedure B (Optimization Table B)}

A screw-top test tube equipped with a stir bar was charged with the photocatalyst and (2bromopyridin-3-yl)(1H-indol-1-yl)methanone (1) (30.1 mg, $0.1 \mathrm{mmol}, 1$ equiv). The atmosphere was exchanged three times by applying vacuum and backfilling with $\mathrm{N}_{2}$. Under $\mathrm{N}_{2}$ atmosphere, thiophenol $(0.5 \mu \mathrm{L}, 5 \mathrm{~mol} \%$ ) and amine (3 equiv) were added via microsyringe. Degassed 2,2,2trifluoroethanol was added via syringe and the resulting mixture was stirred for 16 hours under irradiation with blue LEDs. The reaction was diluted with ethyl acetate and filtered through a silica plug which was washed with ethyl acetate. An internal standard of dodecane (10 $\mu \mathrm{L}, 0.044 \mathrm{mmol})$ was added. A sample was analyzed by GC-FID, and the integral values were used to calculate the data given in Table 3 .

\section{II-C: Gas Chromatography Method Conditions:}

The gas chromatography system hardware are reported in section I-B, General Analytical Information. The injection volume for each trial is $0.5 \mu \mathrm{L}$. The initial oven temperature was set to $50{ }^{\circ} \mathrm{C}$, and the ramp rate was programmed to $20^{\circ} \mathrm{C} / \mathrm{min}$ until reaching $150{ }^{\circ} \mathrm{C}$. With no hold time, the temperature ramp rate is adjusted to $25^{\circ} \mathrm{C} / \mathrm{min}$ until reaching the maximum temperature of $325{ }^{\circ} \mathrm{C}$. Maximum temperature is held for one minute before concluding the run. Using this method, the retention times were $11.04 \mathrm{~min}$ for (2-bromopyridin-3-yl)(1H-indol-1-yl)methanone (1), $10.46 \mathrm{~min}$ for 11,11a-dihydro-5H-pyrido[2',3':3,4]pyrrolo[1,2-a]indol-5-one (2), $10.09 \mathrm{~min}$ for (1H-indol-1-yl)(pyridin-3-yl)methanone (HDH), and $10.56 \mathrm{~min}$ for $5 \mathrm{H}$ pyrido[2',3':3,4]pyrrolo[1,2-a]indol-5-one (Heck). The retention time for the dodecane standard was $4.93 \mathrm{~min}$. 


\section{II-D: Optimization Table $\mathbf{A}^{\mathrm{a}}$}

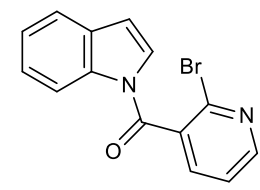

1

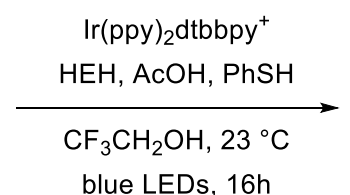

blue LEDs, $16 \mathrm{~h}$

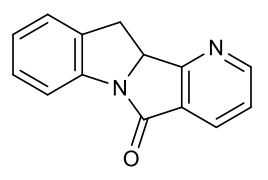

2
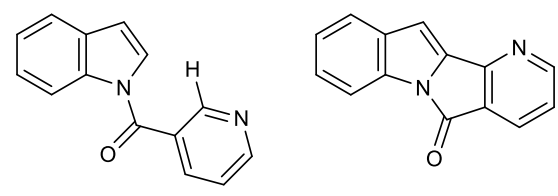

HDH

Heck

\begin{tabular}{cccccccc}
\hline Entry & HEH equiv & Conc. $(\mathbf{M})$ & AcOH equiv & $\mathbf{1}^{\mathbf{b}}$ & $\mathbf{2}^{\mathbf{b}}$ & $\mathbf{H D H}^{\mathbf{b}}$ & $\mathbf{H e c k}^{\mathbf{b}}$ \\
\hline 1 & 1.3 & 0.1 & 2.0 & 31 & 40 & 12 & 10 \\
2 & 2.0 & 0.1 & 2.0 & 25 & 59 & 14 & 5 \\
3 & 3.0 & 0.1 & 2.0 & 4 & 62 & 25 & 3 \\
4 & 5.0 & 0.1 & 2.0 & 1 & 57 & 45 & 4 \\
5 & 3.0 & 0.02 & 2.0 & 5 & 72 & 6 & 13 \\
6 & 3.0 & 0.02 & 20 & 0 & 77 & 7 & 7
\end{tabular}

${ }^{a}$ Conditions: $1(0.1 \mathrm{mmol}),\left[\operatorname{Ir}(\mathrm{ppy})_{2} \mathrm{dtbbpy}_{\mathrm{P}} \mathrm{PF}_{6}(1.0 \mathrm{~mol} \%)\right.$, thiophenol $(5 \mathrm{~mol} \%)$, acetic acid, 2,2,2-trifluoroethanol, blue LEDs, $23{ }^{\circ} \mathrm{C}, 16 \mathrm{~h}$. ${ }^{\mathrm{b}}$ Percent yields determined by GC.

\section{II-E: Optimization Table $B^{a}$}

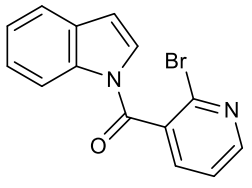

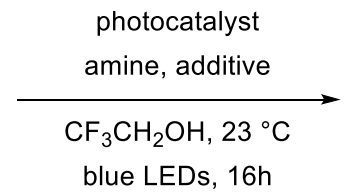

blue LEDs, $16 \mathrm{~h}$

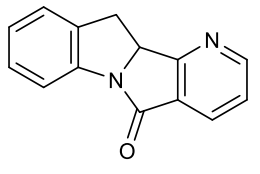

2

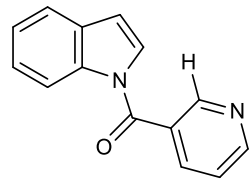

HDH

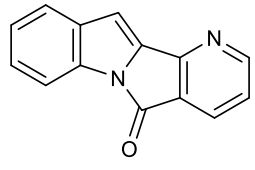

Heck

\begin{tabular}{ccccccccc}
\hline Entry & Amine & Conc. (M) & Additive & Photocatalyst & $\mathbf{1}^{\mathbf{c}}$ & $\mathbf{2}^{\mathbf{c}}$ & HDH $^{\mathbf{c}}$ & Heck $^{\mathbf{c}}$ \\
\hline 1 & $\mathrm{Et} \mathrm{N}_{3}$ & 0.02 & $\mathrm{PhSH}$ & $\operatorname{Ir}(\text { ppy })_{2} \mathrm{dtbbpy}^{+}$ & 29 & 24 & 1 & 17 \\
2 & $i$ - $\mathrm{Pr}_{2} \mathrm{NEt}$ & 0.02 & $\mathrm{PhSH}$ & $\operatorname{Ir}(\text { ppy })_{2} \mathrm{dtbbpy}^{+}$ & 4 & 43 & 0 & 25 \\
3 & $\mathrm{Bu} \mathrm{N}$ & 0.02 & $\mathrm{PhSH}$ & $\operatorname{Ir}(\text { ppy })_{2} \mathrm{dtbbpy}^{+}$ & 2 & 59 & 0 & 23 \\
4 & $\mathrm{Bu}_{3} \mathrm{~N}$ & 0.1 & $\mathrm{PhSH}$ & $\operatorname{Ir}(\text { ppy })_{2} \mathrm{dtbbpy}^{+}$ & 1 & 71 & 2 & 13 \\
5 & $\mathrm{Bu}_{3} \mathrm{~N}$ & 0.2 & $\mathrm{PhSH}$ & $\operatorname{Ir}(\text { ppy })_{2} \mathrm{dtbbpy}^{+}$ & 0 & 77 & 3 & 9 \\
6 & $\mathrm{Bu}_{3} \mathrm{~N}$ & 0.2 & - & $\operatorname{Ir}(\text { ppy })_{2} \mathrm{dtbbpy}{ }^{+}$ & 0 & 84 & 2 & 6 \\
$7^{\mathrm{b}}$ & $\mathrm{Bu}_{3} \mathrm{~N}$ & 0.2 & - & $4 \mathrm{CzIPN}$ & 0 & 80 & 2 & 8 \\
$8^{\mathrm{b}}$ & $\mathrm{Bu}_{3} \mathrm{~N}$ & 0.2 & - & 3DPAFIPN & 0 & 83 & 0 & 2
\end{tabular}

${ }^{a}$ Conditions: aryl radical precursor (1 equiv), [Ir(ppy) $\left.{ }_{2} \mathrm{dtbbpy}\right] \mathrm{PF}_{6}(1 \mathrm{~mol} \%)$, thiophenol $(5 \mathrm{~mol}$ $\%)$, 2,2,2-trifluoroethanol $(0.02 \mathrm{M})$, blue LEDs, $23{ }^{\circ} \mathrm{C}, 16 \mathrm{~h} .{ }^{\mathrm{b}} 5 \mathrm{~mol} \%$ photocatalyst. ${ }^{\mathrm{c}}$ Percent yields determined by GC. 


\section{General Procedures}

\section{III-A. Acylation Procedure A}

Adapted from a literature procedure by Umehara, et al. ${ }^{3}$ To an oven-dried round-bottomed flask was added the nicotinic acid derivative ( 1 equiv), indole ( 1 or 2.5 equiv), and DMAP (20 mol\%). The atmosphere was exchanged three times by applying vacuum and backfilling with $\mathrm{N}_{2}$. Acetonitrile $(0.5 \mathrm{M})$ and 2,6-lutidine $(30 \mathrm{~mol} \%)$ were then added via syringe. Di-tert-butyl dicarbonate ( 2.5 or 1.5 equiv) was added portionwise, and the reaction was heated to $30{ }^{\circ} \mathrm{C}$ for 16 24 hours. The reaction was then concentrated in vacuo and purified by column chromatography.

\section{III-B. Acylation Procedure B}

Adapted from a literature procedure by Heller, et $a l .{ }^{4}$ To an oven-dried round-bottomed flask was added the acyl imidazole ( 1.1 equiv). The atmosphere was exchanged three times by applying vacuum and backfilling with $\mathrm{N}_{2}$. Acetonitrile $(0.33 \mathrm{M})$, indole (1 equiv), and DBU (20 mol\%) were added, and the mixture was stirred for 16 hours. The reaction was quenched with saturated aqueous ammonium chloride and extracted three times with ethyl acetate. The combined organic layers were washed two times with brine, filtered through a plug of silica, and concentrated in vacuo. The crude residue was purified by column chromatography, if necessary.

\section{III-C. General Hydroarylation Procedure A}

A $20 \mathrm{~mL}$ screw-top test tube equipped with a stir bar was charged with 3DPAFIPN (16 mg, $5 \mathrm{~mol}$ $\%)$ and the aryl radical precursor ( $0.5 \mathrm{mmol}, 1$ equiv) and sealed with a PTFE/silicon septum. The atmosphere was exchanged three times by applying vacuum and backfilling with $\mathrm{N}_{2}$. Under $\mathrm{N}_{2}$ atmosphere, tributylamine $(0.36 \mathrm{~mL}, 1.5 \mathrm{mmol}, 3$ equiv) and degassed $25 \%$ (v/v) toluene/2,2,2trifluoroethanol $(5 \mathrm{~mL}, 0.1 \mathrm{M})$ were added via syringe. The resulting mixture was stirred for 16 hours under irradiation with blue LEDs. The reaction was concentrated in vacuo. The crude residue was purified by column chromatography.

\section{III-D. General Hydroarylation Procedure B}

A $20 \mathrm{~mL}$ screw-top test tube equipped with a stir bar was charged with 4CzIPN (20 mg, $5 \mathrm{~mol} \%$ ) and the aryl radical precursor (1 equiv) and sealed with a PTFE/silicon septum. The atmosphere was exchanged three times by applying vacuum and backfilling with $\mathrm{N}_{2}$. Under $\mathrm{N}_{2}$ atmosphere, tributylamine (3 equiv) and degassed 2,2,2-trifluoroethanol $(0.1 \mathrm{M})$ were added via syringe. The resulting mixture was stirred for 16 hours at $40{ }^{\circ} \mathrm{C}$ under irradiation with blue LEDs. The reaction was concentrated in vacuo. The crude residue was purified by column chromatography.

\footnotetext{
${ }^{3}$ Umehara, A.; Ueda, H.; Tokuyama, H. Condensation of Carboxylic Acids with Non-Nucleophilic $N$-Heterocycles and Anilides Using $\mathrm{Boc}_{2} \mathrm{O}$. J. Org. Chem. 2016, 81, 11444-11453.

${ }^{4}$ Heller, S. T.; Schultz, E. E.; Sarpong, R. Chemoselective N-Acylation of Indoles and Oxazolidinones with Carbonylazoles. Angew. Chem. Int. Ed. 2012, 51, 8304-8308.
} 


\section{Computational Details}

All DFT calculations were carried out using the Gaussian 9 software package ${ }^{5}$ at the (U)B3LYP or R(B3LYP $)^{5}$ level of theory with the $6-311+\mathrm{G}(\mathrm{d}, \mathrm{p})^{7}$ basis set. The CPCM formalism for the Self Consistent Reaction Field (SCRF) model of solvation was employed in calculations to account for solvation in $\mathrm{MeCN}$, and the default parameters as implemented in Gaussian were used.

Reduction potentials were calculated using a modified procedure as described by Nicewicz and coworkers. ${ }^{8}$ Geometry optimizations were carried out for the reduced and neutral forms of each molecule, and frequency calculations were performed on the minimized structures to ensure no imaginary frequencies existed. Gibbs free energies $\left(\mathrm{G}_{298}\right)$ were obtained from the calculation and employed in the following equation:

$$
E_{1 / 2}^{0, \text { calc }}=-\frac{\left(G_{298}[\text { reduced }]-G_{298}[\text { oxidized }]\right)}{n_{e} \mathcal{F}}-E_{1 / 2}^{0, S H E}+E_{1 / 2}^{0, S C E}
$$

Where $n_{e}$ is the number of electrons transferred $\left(n_{e}=1\right.$ for all calculations here), $\mathcal{F}$ is the Faraday constant (value $23.061 \mathrm{kcal} \mathrm{mol}^{-1} \mathrm{~V}^{-1}$ ), $E_{1 / 2}^{0, S H E}$ is the absolute value for the standard hydrogen electrode (SHE, value $=4.281 \mathrm{~V}$ ) and $E_{1 / 2}^{0, S C E}$ is the potential of the saturated calomel electrode $(\mathrm{SCE})$ relative to the $\mathrm{SHE}$ in $\mathrm{MeCN}$ (value $=-0.141 \mathrm{~V}$ ) ${ }^{9}$, and $\mathrm{G}_{298}$ [oxidized] and $\mathrm{G}_{298}$ [reduced] are the Gibbs free energies in MeCN obtained from DFT calculations.

\footnotetext{
${ }^{5}$ M. J. Frisch, G. W. Trucks, H. B. Schlegel, G. E. Scuseria, M. A. Robb, J. R. Cheeseman, G. Scalmani, V. Barone, G. A. Petersson, H. Nakatsuji, X. Li, M. Caricato, A. Marenich, J. Bloino, B. G. Janesko, R. Gomperts, B.

Mennucci, H. P. Hratchian, J. V. Ortiz, A. F. Izmaylov, J. L. Sonnenberg, D. Williams-Young, F. Ding, F. Lipparini, F. Egidi, J. Goings, B. Peng, A. Petrone, T. Henderson, D. Ranasinghe, V. G. Zakrzewski, J. Gao, N. Rega, G. Zheng, W. Liang, M. Hada, M. Ehara, K. Toyota, R. Fukuda, J. Hasegawa, M. Ishida, T. Nakajima, Y. Honda, O. Kitao, H. Nakai, T. Vreven, K. Throssell, J. A. Montgomery, Jr., J. E. Peralta, F. Ogliaro, M. Bearpark, J. J. Heyd, E. Brothers, K. N. Kudin, V. N. Staroverov, T. Keith, R. Kobayashi, J. Normand, K. Raghavachari, A. Rendell, J. C. Burant, S. S. Iyengar, J. Tomasi, M. Cossi, J. M. Millam, M. Klene, C. Adamo, R. Cammi, J. W. Ochterski, R. L. Martin, K. Morokuma, O. Farkas, J. B. Foresman, and D. J. Fox, Gaussian, Inc., Wallingford CT, 2016.

${ }^{6}$ (a) Lee, C.; Yang, W.; Parr, R. G. Phys. Rev. B, 1988, 37 (2), 785-789. (b) Becke, A. D. J. Chem. Phys. 1993, 98 (7), 5648-5652.

${ }^{7}$ McLean, A. D.; Chandler, G. S. Contracted Gaussian Basis Sets for Molecular Calculations. I. Second Row Atoms, Z=11-18. J. Chem. Phys. 1980, 72, 5639- 5648.

${ }^{7}$ Roth, H. G.; Romero, N. A.; Nicewicz, D. A. Experimental and Calculated Electrochemical Potentials of Common Organic Molecules for Applications to Single Electron Redox Chemistry. Synlett 2016, 27 (05), 714-723.

${ }^{8}$ Isse, A. A.; Gennaro, A. Absolute Potential of the Standard Hydrogen Electrode and the Problem of Interconversion of Potentials in Different Solvents. J. Phys. Chem. B 2010, 114 (23), 7894-7899.

${ }^{9}$ Roy Dennington TK and JM. GaussView, Version 5, Semichem Inc, Shawnee Mission KS, 2009.
} 
Molecular coordinates of optimized structures:<smiles>O=C1C2c3ncccc3C(=O)C23C(=O)c2ccccc2N13</smiles>

Charge: 0

Multiplicity: 2

Number of imaginary frequencies: 0

Solvation: $\mathrm{MeCN}$

$\mathrm{G}_{298}=-723.646901$ Hartree

$\begin{array}{lrrr}\mathrm{C} & 6.01047210 & 3.84282338 & -3.36995921 \\ \mathrm{C} & 6.82220823 & 2.97977439 & -2.67728796 \\ \mathrm{C} & 8.08541859 & 3.36146323 & -2.23789742 \\ \mathrm{C} & 8.46426425 & 4.70849038 & -2.42902512 \\ \mathrm{C} & 7.56603969 & 5.62933981 & -3.03540245 \\ \mathrm{C} & 6.30993608 & 5.19277183 & -3.50785493 \\ \mathrm{H} & 8.74260047 & 2.66243554 & -1.76421792 \\ \mathrm{H} & 9.43069851 & 5.03945544 & -2.11064337 \\ \mathrm{H} & 7.84513866 & 6.65732090 & -3.13668740 \\ \mathrm{H} & 5.61942585 & 5.87238365 & -3.96197320 \\ \mathrm{C} & 4.79669352 & 3.04643061 & -3.81808263 \\ \mathrm{H} & 4.22666066 & 3.14278913 & -4.71845898 \\ \mathrm{C} & 4.64957221 & 2.00719387 & -2.72813051 \\ \mathrm{H} & 4.09720654 & 2.40388300 & -1.90203736 \\ \mathrm{~N} & 6.09953308 & 1.69242210 & -2.46169951 \\ \mathrm{C} & 6.38454962 & 0.63151814 & -3.44197989 \\ \mathrm{C} & 4.05499899 & 0.68589768 & -3.25273266 \\ \mathrm{C} & 5.04433241 & -0.05579219 & -3.74722429 \\ \mathrm{O} & 7.50777597 & 0.33921303 & -3.92828314 \\ \mathrm{~N} & 2.65646019 & 0.24545277 & -3.26903460 \\ \mathrm{C} & 4.76342039 & -1.35965441 & -4.48138328 \\ \mathrm{H} & 5.54789933 & -1.93732227 & -4.92386790 \\ \mathrm{C} & 3.46456714 & -1.75383803 & -4.53990342 \\ \mathrm{H} & 3.20084730 & -2.66041368 & -5.04336388 \\ \mathrm{C} & 2.35938328 & -0.88224011 & -3.86685510 \\ \mathrm{H} & 1.34119034 & -1.20990225 & -3.89550214\end{array}$


<smiles>O=C1c2cccnc2C2(O)c3ccccc3N12</smiles>

Charge: -1

Multiplicity: 1

Number of imaginary frequencies: 0

Solvation: $\mathrm{MeCN}$

$\mathrm{G}_{298}=-723.763927$ Hartree

$\begin{array}{lrrr}\mathrm{C} & 6.01047210 & 3.84282338 & -3.36995921 \\ \mathrm{C} & 6.82220823 & 2.97977439 & -2.67728796 \\ \mathrm{C} & 8.08541859 & 3.36146323 & -2.23789742 \\ \mathrm{C} & 8.46426425 & 4.70849038 & -2.42902512 \\ \mathrm{C} & 7.56603969 & 5.62933981 & -3.03540245 \\ \mathrm{C} & 6.30993608 & 5.19277183 & -3.50785493 \\ \mathrm{H} & 8.74260047 & 2.66243554 & -1.76421792 \\ \mathrm{H} & 9.43069851 & 5.03945544 & -2.11064337 \\ \mathrm{H} & 7.84513866 & 6.65732090 & -3.13668740 \\ \mathrm{H} & 5.61942585 & 5.87238365 & -3.96197320 \\ \mathrm{C} & 4.79669352 & 3.04643061 & -3.81808263 \\ \mathrm{H} & 4.22666066 & 3.14278913 & -4.71845898 \\ \mathrm{C} & 4.64957221 & 2.00719387 & -2.72813051 \\ \mathrm{H} & 4.09720654 & 2.40388300 & -1.90203736 \\ \mathrm{~N} & 6.09953308 & 1.69242210 & -2.46169951 \\ \mathrm{C} & 6.38454962 & 0.63151814 & -3.44197989 \\ \mathrm{C} & 4.05499899 & 0.68589768 & -3.25273266 \\ \mathrm{C} & 5.04433241 & -0.05579219 & -3.74722429 \\ \mathrm{O} & 7.50777597 & 0.33921303 & -3.92828314 \\ \mathrm{~N} & 2.65646019 & 0.24545277 & -3.26903460 \\ \mathrm{C} & 4.76342039 & -1.35965441 & -4.48138328 \\ \mathrm{H} & 5.54789933 & -1.93732227 & -4.92386790 \\ \mathrm{C} & 3.46456714 & -1.75383803 & -4.53990342 \\ \mathrm{H} & 3.20084730 & -2.66041368 & -5.04336388 \\ \mathrm{C} & 2.35938328 & -0.88224011 & -3.86685510 \\ \mathrm{H} & 1.34119034 & -1.20990225 & -3.89550214\end{array}$




\section{Preparation of Starting Materials}<smiles>O=C(c1cccnc1Br)n1ccnc1</smiles>

(2-bromopyridin-3-yl)(1H-imidazol-1-yl)methanone (S1): CDI (8.92 g, 55 mmol) was added to a stirred solution of 2-bromonicotinic acid (10.1 g, $50 \mathrm{mmol})$ in THF (100 mL). After 15 hours, the solvent was removed in vacuo. The resulting residue was dissolved in DCM, washed with water and brine, dried over $\mathrm{MgSO}_{4}$, filtered, and concentrated in vacuo to obtain the title compound (9.6 g, 76\%) as a yellow, viscous oil.

${ }^{1} \mathbf{H}$ NMR $\left(600 \mathrm{MHz}, \mathrm{CDCl}_{3}\right) \delta 8.34(\mathrm{ddd}, J=4.8,2.0,0.6 \mathrm{~Hz}, 1 \mathrm{H}), 7.69$ (ddd, $J=7.6,2.0,0.6$ $\mathrm{Hz}, 1 \mathrm{H}), 7.67(\mathrm{~s}, 1 \mathrm{H}), 7.32(\mathrm{ddd}, J=7.6,4.8,0.6 \mathrm{~Hz}, 1 \mathrm{H}), 7.20(\mathrm{t}, J=1.6 \mathrm{~Hz}, 1 \mathrm{H}), 6.88(\mathrm{dd}, J=$ $1.8,0.8 \mathrm{~Hz}, 1 \mathrm{H}) \mathrm{ppm}$.

${ }^{13}$ C NMR (126 MHz, $\left.\mathrm{CDCl}_{3}\right) \delta 162.8,152.5,138.2,137.8,137.5,131.7,122.8,116.8 \mathrm{ppm}$.

FTIR (neat) $v_{\max }: 3126,3050,2989,1718,1575,1555,1470,1395,1374,1306,1290,1241,1216$, 1183, 1093, 1079, 1050, 1012, 893, 811, 747, 718, 643, $594 \mathrm{~cm}^{-1}$.

HRMS (APCI) $m / z$ : [M+H] $]^{+}$calcd. for $\mathrm{C}_{9} \mathrm{H}_{7} \mathrm{ON}_{3} \mathrm{Br}, 251.9767$; found, 251.9766 .

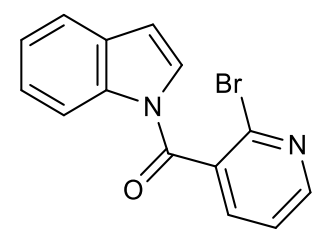

(2-bromopyridin-3-yl)(1H-indol-1-yl)methanone (1): Following the general acylation procedure $\mathrm{A}$, the reaction of 2-bromonicotinic acid (2.02 g, $10 \mathrm{mmol}, 1$ equiv), indole ( $2.93 \mathrm{~g}, 25$ mmol, 2.5 equiv), DMAP (0.244 g, $2.0 \mathrm{mmol}, 20 \mathrm{~mol} \%$ ), 2,6-lutidine (0.35 mL, $3.0 \mathrm{mmol}, 30$ mol\%), and di-tert-butyl dicarbonate (5.46 g, $25 \mathrm{mmol}, 2.5$ equiv) provided the title compound $(2.12 \mathrm{~g}, 71 \%)$ as a white amorphous solid after purification by silica gel chromatography $(20-60 \%$ EtOAc/Hex).

${ }^{1} \mathbf{H}$ NMR $\left(600 \mathrm{MHz}, \mathrm{CDCl}_{3}, 59{ }^{\circ} \mathrm{C}\right) \delta 8.56(\mathrm{dd}, J=4.8,1.9 \mathrm{~Hz}, 1 \mathrm{H}), 8.37(\mathrm{~s}, 1 \mathrm{H}), 7.78(\mathrm{dd}, J=$ 7.6, 1.9 Hz, 1H), 7.59 (d, $J=7.7 \mathrm{~Hz}, 1 \mathrm{H}), 7.44$ (dd, $J=7.4,4.7 \mathrm{~Hz}, 1 \mathrm{H}), 7.40$ (t, $J=7.7 \mathrm{~Hz}, 1 \mathrm{H})$, $7.34(\mathrm{td}, J=7.5,1.2 \mathrm{~Hz}, 1 \mathrm{H}), 6.95(\mathrm{~s}, 1 \mathrm{H}), 6.64(\mathrm{~d}, J=3.8 \mathrm{~Hz}, 1 \mathrm{H}) \mathrm{ppm}$.

${ }^{13} \mathrm{C}$ NMR $\left(126 \mathrm{MHz}, \mathrm{CDCl}_{3}\right){ }^{13} \mathrm{C}$ NMR $(126 \mathrm{MHz}$, Chloroform- $d) \delta 164.9,151.8,138.9,137.4,135.6$, $134.4,131.2,126.2,125.8,124.9,122.9,121.3,116.7,110.8 \mathrm{ppm}$.

FTIR (neat) $v_{\max }: 3116,3034,1680,1605,1554,1543,1449,1395,1381,1350,1204,1187,1100$, $1083,1049,1015,885,871,808,768,744,725,655,625,613 \mathrm{~cm}^{-1}$.

HRMS (APCI) $m / z$ : $[\mathrm{M}+\mathrm{H}]^{+}$calcd. for $\mathrm{C}_{14} \mathrm{H}_{10} \mathrm{ON}{ }_{2} \mathrm{Br}, 300.9971$; found, 300.9972 . 


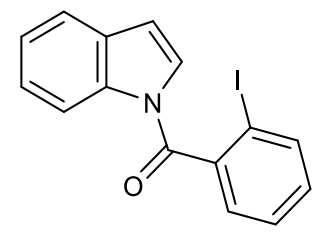

(1H-indol-1-yl)(2-iodophenyl)methanone (S2): CDI (1.78 g, $11 \mathrm{mmol})$ was added to a stirred solution of 2-iodobenzoic acid $(2.48 \mathrm{~g}, 10 \mathrm{mmol})$ in THF $(20 \mathrm{~mL})$. After 16 hours, the solvent was removed in vacuo. The resulting residue was dissolved in DCM, washed with water and brine, dried over $\mathrm{MgSO}_{4}$, filtered, and concentrated in vacuo to obtain crude (1H-imidazol-1-yl)(2iodophenyl)methanone $(2.39 \mathrm{~g}, 80 \%)$ as a yellow oil. Following the general acylation procedure $\mathrm{B}$, the reaction of (1H-imidazol-1-yl)(2-iodophenyl)methanone $(1.97 \mathrm{~g}, 6.6 \mathrm{mmol}, 1.1$ equiv), indole $(0.70 \mathrm{~g}, 6.0 \mathrm{mmol}, 1$ equiv), and DBU $(0.18 \mathrm{~mL}, 1.0 \mathrm{mmol}, 20 \mathrm{~mol} \%)$ provided the title compound $(2.04 \mathrm{~g}, 98 \%)$ as a white amorphous solid after purification by silica gel chromatography (10-30\% EtOAc/Hex).

${ }^{1} \mathbf{H}$ NMR $\left(600 \mathrm{MHz}, \mathrm{CDCl}_{3}, 50{ }^{\circ} \mathrm{C}\right) \delta 8.42(\mathrm{~s}, 1 \mathrm{H}), 7.94(\mathrm{~d}, J=7.9 \mathrm{~Hz}, 1 \mathrm{H}), 7.61(\mathrm{dd}, J=7.9,1.2$ $\mathrm{Hz}, 1 \mathrm{H}), 7.48(\mathrm{t}, J=7.5 \mathrm{~Hz}, 1 \mathrm{H}), 7.46-7.39(\mathrm{~m}, 2 \mathrm{H}), 7.35(\mathrm{t}, J=7.5 \mathrm{~Hz}, 1 \mathrm{H}), 7.22(\mathrm{t}, J=7.8 \mathrm{~Hz}$, $1 \mathrm{H}), 6.98(\mathrm{~s}, 1 \mathrm{H}), 6.62(\mathrm{~d}, J=3.8 \mathrm{~Hz}, 1 \mathrm{H}) \mathrm{ppm}$.

${ }^{13}$ C NMR $\left(126 \mathrm{MHz}, \mathrm{CDCl}_{3}\right) \delta 168.0,141.0,139.5,135.5,131.6,131.1,128.3,128.3,126.8,125$. $3,124.4,121.1,116.5,109.8,92.5 \mathrm{ppm}$.

FTIR (neat) $v_{\max }: 3151,3116,3053,3034,1679,1541,1447,1431,1379,1346,1203,1187,1150$, $1075,1015,886,856,774,744,725,717,690,647,626 \mathrm{~cm}^{-1}$.

HRMS (APCI) $m / z:[\mathrm{M}+\mathrm{H}]^{+}$calcd. for $\mathrm{C}_{15} \mathrm{H}_{11} \mathrm{ONI}, 347.9880$; found, 347.9880 .

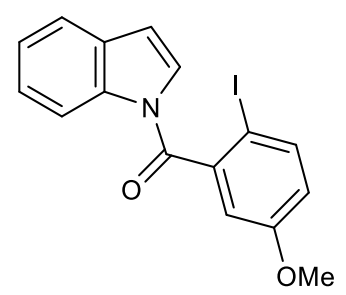

(1H-indol-1-yl)(2-iodo-5-methoxyphenyl)methanone (S3): CDI (268 mg, $1.65 \mathrm{mmol}$ ) was added to a stirred solution of 2-iodo-5-methoxybenzoic acid $(417 \mathrm{mg}, 1.5 \mathrm{mmol})$ in THF (5 mL). After 1 hour, the solvent was removed in vacuo. The resulting residue was dissolved in DCM, washed with brine, filtered through a plug of silica, and concentrated in vacuo to obtain crude $(1 \mathrm{H}-$ imidazol-1-yl)(2-iodo-5-methoxyphenyl)methanone $(431 \mathrm{mg}, 88 \%)$ as a white amorphous solid. Following the general acylation procedure $\mathrm{B}$, the reaction of $(1 \mathrm{H}$-imidazol-1-yl)(2-iodo-5methoxyphenyl)methanone ( $427 \mathrm{mg}, 1.3 \mathrm{mmol}, 1.1$ equiv), indole ( $141 \mathrm{mg}, 1.2 \mathrm{mmol}, 1.0$ equiv), and DBU (36 $\mu \mathrm{L}, 0.24 \mathrm{mmol}, 20 \mathrm{~mol} \%)$ provided the title compound (346 $\mathrm{mg}, 76 \%$ ) as a white amorphous solid after purification by silica gel chromatography $(30-50 \% \mathrm{EtOAc} / \mathrm{Hex})$.

${ }^{1} \mathbf{H}$ NMR $\left(500 \mathrm{MHz}, \mathrm{CDCl}_{3}, 50{ }^{\circ} \mathrm{C}\right) \delta 8.37(\mathrm{~s}, 1 \mathrm{H}), 7.78(\mathrm{~d}, J=8.8 \mathrm{~Hz}, 1 \mathrm{H}), 7.59(\mathrm{dt}, J=7.7,1.1$ $\mathrm{Hz}, 1 \mathrm{H}), 7.39(\mathrm{t}, J=7.7 \mathrm{~Hz}, 1 \mathrm{H}), 7.33(\mathrm{td}, J=7.5,1.1 \mathrm{~Hz}, 1 \mathrm{H}), 7.00(\mathrm{~m}, 2 \mathrm{H}), 6.82(\mathrm{dd}, J=8.8$, $3.0 \mathrm{~Hz}, 1 \mathrm{H}), 6.62(\mathrm{~d}, J=3.7 \mathrm{~Hz}, 1 \mathrm{H}), 3.82(\mathrm{~s}, 3 \mathrm{H}) \mathrm{ppm}$.

${ }^{13} \mathrm{C}$ NMR $\left(126 \mathrm{MHz}, \mathrm{CDCl}_{3}\right) \delta 167.9,160.0,142.0,140.4,135.6,131.3,126.9,125.4,124.5$, $121.1,118.4,116.7,114.2,110.0,80.8,55.8 \mathrm{ppm}$.

FTIR (neat) $v_{\max }: 3151,3107,3872,2942,2847,1685,1583,1535,1467,1448,1391,1377,1342$, $1301,1227,1204,1174,1073,1030,1006,907,859,818,751,724,632,580 \mathrm{~cm}^{-1}$.

HRMS (APCI) $m / z$ : $[\mathrm{M}+\mathrm{H}]^{+}$calcd. for $\mathrm{C}_{16} \mathrm{H}_{13} \mathrm{O}_{2} \mathrm{NI}$, 377.9986; found, 377.9983 . 


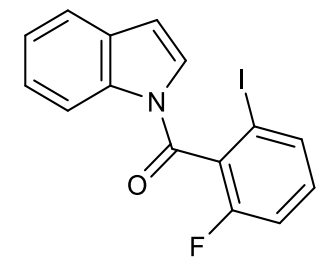

(2-fluoro-6-iodophenyl)(1H-indol-1-yl)methanone (S4): CDI (0.892 g, 5.5 mmol) was added to a stirred solution of 2-fluoro-6-iodobenzoic acid (1.33 g, $5 \mathrm{mmol})$ in THF (10 mL). After 1 hour, the solvent was removed in vacuo. The resulting residue was dissolved in DCM, washed with brine, filtered through a plug of silica, and concentrated in vacuo to obtain crude (2-fluoro-6iodophenyl)(1H-imidazol-1-yl)methanone $(0.899 \mathrm{~g}, 57 \%)$ as a light yellow amorphous solid. Following the general acylation procedure B, the reaction of (2-fluoro-6-iodophenyl)(1Himidazol-1-yl)methanone (695 mg, $2.2 \mathrm{mmol}, 1.1$ equiv), indole (234 mg, $2.0 \mathrm{mmol}, 1$ equiv), and DBU (60 $\mu \mathrm{L}, 0.4 \mathrm{mmol}, 20 \mathrm{~mol} \%$ ) provided the title compound (489 $\mathrm{mg}, 67 \%$ ) as a white amorphous solid after purification by silica gel chromatography (5-10\% EtOAc/Hex).

${ }^{1} \mathbf{H}$ NMR $\left(600 \mathrm{MHz}, \mathrm{CDCl}_{3}, 50{ }^{\circ} \mathrm{C}\right) \delta 8.64(\mathrm{~d}, J=8.2 \mathrm{~Hz}, 1 \mathrm{H}), 7.74(\mathrm{t}, J=4.5 \mathrm{~Hz}, 1 \mathrm{H}), 7.59(\mathrm{dd}$, $J=7.9,1.1 \mathrm{~Hz}, 1 \mathrm{H}), 7.45(\mathrm{t}, J=7.5 \mathrm{~Hz}, 1 \mathrm{H}), 7.35$ (t, $J=7.4 \mathrm{~Hz}, 1 \mathrm{H}), 7.28-7.20(\mathrm{~m}, 2 \mathrm{H}), 6.86$ $(\mathrm{s}, 1 \mathrm{H}), 6.63(\mathrm{~s}, 1 \mathrm{H}) \mathrm{ppm}$.

${ }^{13}$ C NMR $\left(126 \mathrm{MHz}, \mathrm{CDCl}_{3}\right) \delta 163.8,158.8\left(\mathrm{~d},{ }^{1} J_{\mathrm{C}-\mathrm{F}}=254.1 \mathrm{~Hz}\right), 135.6,135.3,135.2,133.0(\mathrm{~d}$, $\left.{ }^{2} J_{\mathrm{C}-\mathrm{F}}=8.2 \mathrm{~Hz}\right), 131.3,126.1,125.7,124.9,121.2,117.1,116.1\left(\mathrm{~d},{ }^{2} J_{\mathrm{C}-\mathrm{F}}=20.8 \mathrm{~Hz}\right), 110.6,93.2(\mathrm{~d}$, $\left.{ }^{3} J_{\mathrm{C}-\mathrm{F}}=2.4 \mathrm{~Hz}\right) \mathrm{ppm}$.

${ }^{19}$ F NMR $\left(376 \mathrm{MHz}, \mathrm{CDCl}_{3}\right) \delta-109.9(\mathrm{t}, J=7.2 \mathrm{~Hz}) \mathrm{ppm}$.

FTIR (neat) $v_{\max }: 3147,3122,3070,3052,3034,1686,1596,1562,1541,1447,1379,1346,1244$, 1236, 1203, 1149, 1124, 1072, 885, 872, 851, 788, 767, 741, 718, 688, $637 \mathrm{~cm}^{-1}$.

HRMS (APCI) $m / z$ : $[\mathrm{M}+\mathrm{H}]^{+}$calcd. for $\mathrm{C}_{15} \mathrm{H}_{10} \mathrm{ONFI}$, 365.9786; found, 365.9786 .

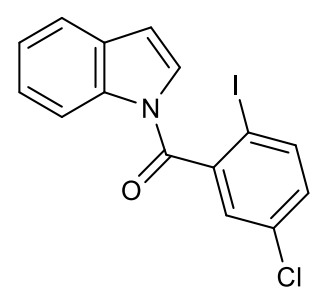

(5-chloro-2-iodophenyl)(1H-indol-1-yl)methanone (S5): CDI (0.892 g, 5.5 mmol) was added to a stirred solution of 5-chloro-2-iodobenzoic acid (1.41 g, $5 \mathrm{mmol})$ in THF $(10 \mathrm{~mL})$. After 1 hour, the solvent was removed in vacuo. The resulting residue was dissolved in DCM, washed with brine, filtered through a plug of silica, and concentrated in vacuo to obtain crude (5-chloro-2iodophenyl)(1H-imidazol-1-yl)methanone $(1.35 \mathrm{~g}, 81 \%)$ as a yellow amorphous solid. Following the general acylation procedure $\mathrm{B}$, the reaction of (5-chloro-2-iodophenyl)(1H-imidazol-1yl)methanone (732 mg, $2.2 \mathrm{mmol}, 1.1$ equiv), indole (234 mg, $2.0 \mathrm{mmol}, 1$ equiv), and DBU (60 $\mu \mathrm{L}, 0.4 \mathrm{mmol}, 20 \mathrm{~mol} \%$ ) provided the title compound (719 $\mathrm{mg}, 94 \%)$ as a white amorphous solid. ${ }^{1} \mathbf{H}$ NMR $\left(600 \mathrm{MHz}, \mathrm{CDCl}_{3}, 50{ }^{\circ} \mathrm{C}\right) \delta 8.36(\mathrm{~s}, 1 \mathrm{H}), 7.86(\mathrm{~d}, J=8.5 \mathrm{~Hz}, 1 \mathrm{H}), 7.59(\mathrm{~d}, J=7.8 \mathrm{~Hz}$, $1 \mathrm{H}), 7.44(\mathrm{~s}, 1 \mathrm{H}), 7.40(\mathrm{t}, J=7.7 \mathrm{~Hz}, 1 \mathrm{H}), 7.34(\mathrm{t}, J=7.5 \mathrm{~Hz}, 1 \mathrm{H}), 7.23(\mathrm{dt}, J=8.5,2.3 \mathrm{~Hz}, 1 \mathrm{H})$, $6.94(\mathrm{~s}, 1 \mathrm{H}), 6.64(\mathrm{~d}, J=3.8 \mathrm{~Hz}, 1 \mathrm{H}) \mathrm{ppm}$.

${ }^{13} \mathrm{C}$ NMR $\left(126 \mathrm{MHz}, \mathrm{CDCl}_{3}\right) \delta 166.7,142.7,140.9,135.5,135.2,132.0,131.2,128.6,126.5$, $125.7,124.8,121.3,116.7,110.5,89.8 \mathrm{ppm}$. 
FTIR (neat) $v_{\max }: 3153,3128,3110,3073,3052,1689,1541,1450,1384,1343,1232,1206,1185$, 1149, 1096, 1074, 1012, 907, 882, 809, 779, 767, 754, 746, 723, 632, $617 \mathrm{~cm}^{-1}$.

HRMS (APCI) $m / z$ : [M+H] $]^{+}$calcd. for $\mathrm{C}_{15} \mathrm{H}_{10} \mathrm{ONClI}, 381.9490$; found, 381.9494 .

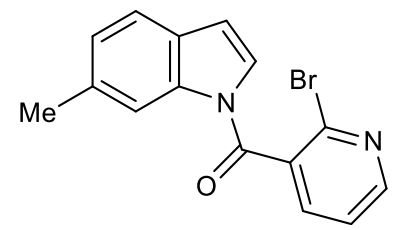

(2-bromopyridin-3-yl)(6-methyl-1H-indol-1-yl)methanone (S6): Following the general acylation procedure $\mathrm{B}$, the reaction of (2-bromopyridin-3-yl)(1H-imidazol-1-yl)methanone (S1 (555 mg, $2.2 \mathrm{mmol}, 1.1$ equiv), 6-methylindole (262 mg, $2.0 \mathrm{mmol}, 1$ equiv), and DBU (60 $\mu \mathrm{L}$, $0.4 \mathrm{mmol}, 20 \mathrm{~mol} \%$ ) provided the title compound (532 $\mathrm{mg}, 84 \%)$ as a white amorphous solid after purification by silica gel chromatography (20\% EtOAc/Hex).

${ }^{1} \mathbf{H}$ NMR $\left(400 \mathrm{MHz}, \mathrm{CDCl}_{3}, 59^{\circ} \mathrm{C}\right) \delta 8.57(\mathrm{dd}, J=4.9,2.0 \mathrm{~Hz}, 1 \mathrm{H}), 8.36(\mathrm{~s}, 1 \mathrm{H}), 7.79(\mathrm{dd}, J=$ $7.5,2.0 \mathrm{~Hz}, 1 \mathrm{H}), 7.50-7.43(\mathrm{~m}, 2 \mathrm{H}), 7.19(\mathrm{dd}, J=7.9,0.8 \mathrm{~Hz}, 1 \mathrm{H}), 6.82(\mathrm{~s}, 1 \mathrm{H}), 6.60(\mathrm{dd}, J=$ $3.8,0.8 \mathrm{~Hz}, 1 \mathrm{H}), 2.52(\mathrm{~s}, 3 \mathrm{H}) \mathrm{ppm}$.

${ }^{13} \mathrm{C}$ NMR $\left(126 \mathrm{MHz}, \mathrm{CDCl}_{3}\right) \delta 164.8,151.5,138.6,137.2,135.8,134.2,128.7,126.2,125.5$, $122.8,120.7,116.9,110.6,22.0 \mathrm{ppm}$.

FTIR (neat) $v_{\max }: 3132,3110,3066,3034,2914,2854,1687,1574,1528,1426,1384,1346,1200$, $1173,1122,1084,1041,884,812,757,711,648,619,596 \mathrm{~cm}^{-1}$.

HRMS (APCI) $m / z$ : [M+H] $]^{+}$calcd. for $\mathrm{C}_{15} \mathrm{H}_{12} \mathrm{ON}_{2} \mathrm{Br}, 315.0128$; found, 315.0125 .<smiles>CCCCOc1cccc2c1ccn2C(=O)c1cccnc1Br</smiles>

(2-bromopyridin-3-yl)(4-((tert-butyldimethylsilyl)oxy)-1H-indol-1-yl)methanone

(S7):

Following the general acylation procedure B, the reaction of (2-bromopyridin-3-yl)(1H-imidazol1-yl)methanone (S1 (832 mg, $3.3 \mathrm{mmol}, 1.1$ equiv), 4-hydroxyindole (399 mg, $3.0 \mathrm{mmol}, 1$ equiv), and DBU $(90 \mu \mathrm{L}, 0.6 \mathrm{mmol}, 20 \mathrm{~mol} \%)$ provided crude (2-bromopyridin-3-yl)(4-hydroxy-1Hindol-1-yl)methanone $(879 \mathrm{mg})$. The crude solid was then dissolved in $\mathrm{MeCN}(10 \mathrm{~mL})$ with imidazole (408 mg, $6.0 \mathrm{mmol})$. TBSCl $(452 \mathrm{mg}, 3.0 \mathrm{mmol})$ was added and the resulting solution was stirred for 20 hours. The reaction was diluted with EtOAc, washed with brine (3x), dried over $\mathrm{MgSO}_{4}$, filtered, and concentrated in vacuo. The title compound was obtained as a white amorphous solid after purification by silica gel chromatography (10-20\% EtOAc/Hex).

${ }^{1} \mathbf{H}$ NMR $\left(500 \mathrm{MHz}, \mathrm{CDCl}_{3}, 50{ }^{\circ} \mathrm{C}\right) \delta 8.57$ (ddd, $\left.J=4.9,2.0,0.9 \mathrm{~Hz}, 1 \mathrm{H}\right), 7.97(\mathrm{~s}, 1 \mathrm{H}), 7.78$ (ddd, $J=7.6,2.0,0.9 \mathrm{~Hz}, 1 \mathrm{H}), 7.45(\mathrm{ddd}, J=7.6,5.0,0.9 \mathrm{~Hz}, 1 \mathrm{H}), 7.25(\mathrm{t}, J=8.2 \mathrm{~Hz}, 1 \mathrm{H}), 6.84(\mathrm{~s}, 1 \mathrm{H})$, $6.77(\mathrm{~d}, J=8.0 \mathrm{~Hz}, 1 \mathrm{H}), 6.71(\mathrm{dt}, J=3.9,0.9 \mathrm{~Hz}, 1 \mathrm{H}), 1.05(\mathrm{~s}, 9 \mathrm{H}), 0.26(\mathrm{~s}, 6 \mathrm{H}) \mathrm{ppm}$.

${ }^{13}$ C NMR $\left(126 \mathrm{MHz}, \mathrm{CDCl}_{3}\right) \delta 164.9,151.7,149.0,138.8,137.3,137.1,134.4,126.6,124.7$, 124. $2,122.9,114.4,110.0,108.1,25.9,18.4,-4.2 \mathrm{ppm}$. 
FTIR (neat) $v_{\max }: 2955,2929,2856,1678,1594,1578,1481,1431,1389,1350,1284,1255,1227$, $1151,1090,1015,868,834,814,779,748,722,684,643 \mathrm{~cm}^{-1}$.

HRMS (APCI) $m / z$ : [M+H] $]^{+}$calcd. for $\mathrm{C}_{20} \mathrm{H}_{24} \mathrm{O}_{2} \mathrm{~N}_{2} \mathrm{BrSi}$, 431.0785; found, 431.0783 .<smiles>COc1ccc2c(ccn2C(=O)c2cccnc2Br)c1</smiles>

(2-bromopyridin-3-yl)(5-methoxy-1H-indol-1-yl)methanone (S8): Following the general acylation procedure $\mathrm{B}$, the reaction of (2-bromopyridin-3-yl)(1H-imidazol-1-yl)methanone (555 $\mathrm{mg}, 2.2 \mathrm{mmol}, 1.1$ equiv), 5-methoxyindole (294 mg, $2.0 \mathrm{mmol}, 1$ equiv), and DBU (60 $\mu \mathrm{L}, 0.4$ mmol, $20 \mathrm{~mol} \%)$ provided the title compound $(655 \mathrm{mg}, 99 \%)$ as a light brown amorphous solid.

${ }^{1} \mathbf{H}$ NMR $\left(500 \mathrm{MHz}, \mathrm{CDCl}_{3}, 59{ }^{\circ} \mathrm{C}\right) \delta 8.53(\mathrm{dd}, J=4.7,1.8 \mathrm{~Hz}, 1 \mathrm{H}), 8.31(\mathrm{~s}, 1 \mathrm{H}), 7.77(\mathrm{dd}, J=$ $7.5,2.0 \mathrm{~Hz}, 1 \mathrm{H}), 7.42(\mathrm{dd}, J=7.5,4.8 \mathrm{~Hz}, 1 \mathrm{H}), 7.05(\mathrm{~d}, J=2.5 \mathrm{~Hz}, 1 \mathrm{H}), 6.99(\mathrm{dd}, J=9.0,2.5 \mathrm{~Hz}$, $1 \mathrm{H}), 6.88(\mathrm{~s}, 1 \mathrm{H}), 6.56(\mathrm{~d}, J=3.8 \mathrm{~Hz}, 1 \mathrm{H}), 3.86(\mathrm{~s}, 3 \mathrm{H}) \mathrm{ppm}$.

${ }^{13}$ C NMR (126 MHz, $\left.\mathrm{CDCl}_{3}\right) \delta 164.4,157.4,151.7,138.8,137.3,134.3,132.2,130.1,126.9$, $122.8,117.4,113.8,110.6,104.2,55.8 \mathrm{ppm}$.

FTIR (neat) $v_{\max }: 3160,3107,3053,3005,2964,2841,1691,1471,1444,1399,1370,1342,1333$, 1274, 1257, 1194, 1182, 1149, 1095, 1051, 1042, 1022, 881, 823, 814, 794, 749, 736, 717, 650, $632,582 \mathrm{~cm}^{-1}$.

HRMS (APCI) $m / z$ : [M+H] $]^{+}$calcd. for $\mathrm{C}_{15} \mathrm{H}_{12} \mathrm{O}_{2} \mathrm{~N}_{2} \mathrm{Br}$, 331.0077; found, 331.0083 .<smiles>COc1ccc2ccn(C(=O)c3cccnc3Br)c2c1</smiles>

(2-bromopyridin-3-yl)(6-methoxy-1H-indol-1-yl)methanone (S9): Following the general acylation procedure $\mathrm{B}$, the reaction of (2-bromopyridin-3-yl)(1H-imidazol-1-yl)methanone (S1 (555 mg, $2.2 \mathrm{mmol}, 1.1$ equiv), 6-methoxyindole (294 mg, $2.0 \mathrm{mmol}, 1$ equiv), and DBU (60 $\mu \mathrm{L}$, $0.4 \mathrm{mmol}, 20 \mathrm{~mol} \%$ ) provided the title compound (549 $\mathrm{mg}, 83 \%)$ as a white amorphous solid after purification by silica gel chromatography (10-30\% EtOAc/Hex).

${ }^{1} \mathbf{H}$ NMR $\left(500 \mathrm{MHz}, \mathrm{CDCl}_{3}, 50{ }^{\circ} \mathrm{C}\right) \delta 8.57(\mathrm{ddd}, J=4.9,2.0,0.8 \mathrm{~Hz}, 1 \mathrm{H}), 8.07(\mathrm{~s}, 1 \mathrm{H}), 7.79$ (ddd, $J=7.4,2.0,0.7 \mathrm{~Hz}, 1 \mathrm{H}), 7.50-7.42(\mathrm{~m}, 2 \mathrm{H}), 6.98$ (ddd, $J=8.6,2.4,0.8 \mathrm{~Hz}, 1 \mathrm{H}), 6.79$ (s, 1H), $6.57(\mathrm{~d}, J=3.7 \mathrm{~Hz}, 1 \mathrm{H}), 3.91(\mathrm{~s}, 3 \mathrm{H}) \mathrm{ppm}$.

${ }^{13} \mathrm{C}$ NMR $\left(126 \mathrm{MHz}, \mathrm{CDCl}_{3}\right) \delta 165.0,158.7,151.6,138.7,137.2,136.5,134.2,124.9,124.6$, $122.8,121.6,113.9,110.5,100.9,55.8 \mathrm{ppm}$.

FTIR (neat) vmax: 3101, 3060, 2946, 2898, 2838, 1682, 1574, 1540, 1479, 1434, 1384, 1352, 1276, $1221,1172,1083,1041,1026,884,866,801,750,708,649,616 \mathrm{~cm}^{-1}$.

HRMS (APCI) $m / z$ : $[\mathrm{M}+\mathrm{H}]^{+}$calcd. for $\mathrm{C}_{15} \mathrm{H}_{12} \mathrm{O}_{2} \mathrm{~N}_{2} \mathrm{Br}, 331.0077$; found, 331.0081 . 
<smiles>O=C(c1cccnc1Br)n1ccc2c(Br)cccc21</smiles>

(4-bromo-1H-indol-1-yl)(2-bromopyridin-3-yl)methanone (S10): Following the general acylation procedure $\mathrm{B}$, the reaction of (2-bromopyridin-3-yl)(1H-imidazol-1-yl)methanone (S1 (555 mg, $2.2 \mathrm{mmol}, 1.1$ equiv), 4-bromoindole (392 mg, $2.0 \mathrm{mmol}, 1$ equiv), and DBU (60 $\mu \mathrm{L}, 0.4$ mmol, $20 \mathrm{~mol} \%$ ) provided the title compound $(647 \mathrm{mg}, 85 \%)$ as a white amorphous solid after purification by silica gel chromatography (10-20\% EtOAc/Hex).

${ }^{1} \mathbf{H}$ NMR $\left(500 \mathrm{MHz}, \mathrm{CDCl}_{3}, 50{ }^{\circ} \mathrm{C}\right) \delta 8.59(\mathrm{dd}, J=4.9,2.0 \mathrm{~Hz}, 1 \mathrm{H}), 8.39$ (s, 1H), 7.80 (ddd, $J=$ 7.6, 2.0, 0.6 Hz, 1H), $7.52(\mathrm{dd}, J=7.9,0.8 \mathrm{~Hz}, 1 \mathrm{H}), 7.48(\mathrm{dd}, J=7.5,4.9 \mathrm{~Hz}, 1 \mathrm{H}), 7.29(\mathrm{t}, J=$ $8.1 \mathrm{~Hz}, 1 \mathrm{H}), 7.00(\mathrm{~s}, 1 \mathrm{H}), 6.74(\mathrm{~d}, J=3.9 \mathrm{~Hz}, 1 \mathrm{H}) \mathrm{ppm}$.

${ }^{13} \mathrm{C}$ NMR $\left(126 \mathrm{MHz}, \mathrm{CDCl}_{3}\right) \delta 165.0,152.0,138.7,137.4,135.8,133.9,131.8,127.8,126.8$, $126.8,122.9,115.7,115.0,110.4 \mathrm{ppm}$.

FTIR (neat) $v_{\max }: 3139,3110,3053,1698,1687,1578,1533,1418,1340,1175,1043,882,765$, $747,560 \mathrm{~cm}^{-1}$.

HRMS (APCI) $m / z$ : $[\mathrm{M}+\mathrm{H}]^{+}$calcd. for $\mathrm{C}_{14} \mathrm{H}_{9} \mathrm{ON}_{2} \mathrm{Br}_{2}, 378.9076$; found, 378.9075 .<smiles>O=C(c1cccnc1Br)n1ccc2cc(Br)ccc21</smiles>

(5-bromo-1H-indol-1-yl)(2-bromopyridin-3-yl)methanone (S11): Following the general acylation procedure $\mathrm{B}$, the reaction of (2-bromopyridin-3-yl)(1H-imidazol-1-yl)methanone (S1 (832 mg, $3.3 \mathrm{mmol}, 1.1$ equiv), 5-bromoindole (588 mg, $3.0 \mathrm{mmol}, 1$ equiv), and DBU (90 $\mu \mathrm{L}, 0.6$ mmol, $20 \mathrm{~mol} \%$ ) provided the title compound $(1.01 \mathrm{~g}, 88 \%)$ as a white amorphous solid after purification by silica gel chromatography (20-30\% EtOAc/Hex).

${ }^{1} \mathbf{H}$ NMR $\left(600 \mathrm{MHz}, \mathrm{CDCl}_{3}, 59^{\circ} \mathrm{C}\right) \delta 8.59(\mathrm{dt}, J=4.9,2.1 \mathrm{~Hz}, 1 \mathrm{H}), 8.36(\mathrm{~s}, 1 \mathrm{H}), 7.81(\mathrm{ddd}, J=$ 7.5, 2.5, 1.9 Hz, 1H), $7.74(\mathrm{t}, J=1.8 \mathrm{~Hz}, 1 \mathrm{H}), 7.53(\mathrm{~d}, J=8.5 \mathrm{~Hz}, 1 \mathrm{H}), 7.51-7.46(\mathrm{~m}, 1 \mathrm{H}), 6.94$ $(\mathrm{s}, 1 \mathrm{H}), 6.60(\mathrm{dd}, J=3.7,1.7 \mathrm{~Hz}, 1 \mathrm{H}) \mathrm{ppm}$.

${ }^{13}$ C NMR $\left(126 \mathrm{MHz}, \mathrm{CDCl}_{3}\right) \delta 164.7,151.9,138.6,137.4,134.1,133.8,132.8,128.4,127.3$, $123.9,122.9,118.1,117.9,109.8 \mathrm{ppm}$.

FTIR (neat) $v_{\max }: 3155,3132,3082,3044,1689,1575,1446,1374,1195,1182,1051,876,803$, $751,728,717,627 \mathrm{~cm}^{-1}$.

HRMS (APCI) $m / z$ : $[\mathrm{M}+\mathrm{H}]^{+}$calcd. for $\mathrm{C}_{14} \mathrm{H}_{9} \mathrm{ON}_{2} \mathrm{Br}_{2}, 378.9076$; found, 378.9083 . 
<smiles>O=C(c1cccnc1Br)n1ccc2ccc(Br)cc21</smiles>

(6-bromo-1H-indol-1-yl)(2-bromopyridin-3-yl)methanone (S12): Following the general acylation procedure $\mathrm{B}$, the reaction of (2-bromopyridin-3-yl)(1H-imidazol-1-yl)methanone (S1 (832 mg, $3.3 \mathrm{mmol}, 1.1$ equiv), 6-bromoindole (588 mg, $3.0 \mathrm{mmol}, 1$ equiv), and DBU (90 $\mu \mathrm{L}, 0.6$ mmol, $20 \mathrm{~mol} \%$ ) provided the title compound $(997 \mathrm{mg}, 87 \%)$ as an off-white amorphous solid. ${ }^{1} \mathbf{H}$ NMR $\left(600 \mathrm{MHz}, \mathrm{CDCl}_{3}, 59^{\circ} \mathrm{C}\right) \delta 8.73(\mathrm{~s}, 1 \mathrm{H}), 8.59(\mathrm{dd}, J=4.9,2.0 \mathrm{~Hz}, 1 \mathrm{H}), 7.81(\mathrm{dd}, J=$ $7.5,2.0 \mathrm{~Hz}, 1 \mathrm{H}), 7.51-7.43(\mathrm{~m}, 3 \mathrm{H}), 6.88(\mathrm{~s}, 1 \mathrm{H}), 6.62(\mathrm{dd}, J=3.8,0.8 \mathrm{~Hz}, 1 \mathrm{H}) \mathrm{ppm}$.

${ }^{13}$ C NMR $\left(126 \mathrm{MHz}, \mathrm{CDCl}_{3}\right) \delta 164.9,152.0,138.8,137.4,136.2,133.9,130.0,128.2,126.7$, $123.0,122.3,120.0,119.5,110.4 \mathrm{ppm}$.

FTIR (neat) $v_{\max }: 3110,3041,2930,1683,1572,1530,1378,1343,1200,1095,1051,1041,1025$, $1005,883,807,750,725,665,636,618,579 \mathrm{~cm}^{-1}$.

HRMS (APCI) $m / z$ : $[\mathrm{M}+\mathrm{H}]^{+}$calcd. for $\mathrm{C}_{14} \mathrm{H}_{9} \mathrm{ON}_{2} \mathrm{Br}_{2}, 378.9076$; found, 378.9082 .<smiles>Brc1ncccc1OCc1ccccc1</smiles>

3-(benzyloxy)-2-bromopyridine (17): A round bottom flask was charged with 2-bromo-3hydroxypyridine $\left(5.0 \mathrm{~g}, 29 \mathrm{mmol}, 1.0\right.$ equiv) and $\mathrm{K}_{2} \mathrm{CO}_{3}(8.0 \mathrm{~g}, 58 \mathrm{mmol}, 2.0$ equiv). DMF was added, followed by benzyl bromide $(3.7 \mathrm{~mL}, 31 \mathrm{mmol}, 1.1$ equiv). The reaction was heated to 80 ${ }^{\circ} \mathrm{C}$ and stirred for $16 \mathrm{~h}$. The reaction was cooled to room temperature and then partitioned between EtOAc and water. The organic layer was washed with brine 3x, dried with $\mathrm{MgSO}_{4}$ and purified on silica gel (20-50\% EtOAc/hexanes eluent) to afford the title compound as a white solid (7.5 g, 99\%).

${ }^{1} \mathbf{H}$ NMR $\left(400 \mathrm{MHz}, \mathrm{CDCl}_{3}\right) \delta 7.96(\mathrm{dd}, J=3.8,2.4 \mathrm{~Hz}, 1 \mathrm{H}), 7.47-7.27(\mathrm{~m}, 5 \mathrm{H}), 7.18-7.09(\mathrm{~m}$, $2 \mathrm{H}), 5.15(\mathrm{~s}, 2 \mathrm{H}) \mathrm{ppm}$.

${ }^{1} \mathrm{H}$ NMR spectrum is consistent with reported values. ${ }^{10}$

\footnotetext{
${ }^{10}$ Flynn, A. R.; McDaniel, K. A.; Hughes, M. E.; Vogt, D. B.; Jui, N. T. Hydroarylation of Arenes via Reductive
} Radical-Polar Crossover. J. Am. Chem. Soc. 2020, 142, 9163-9168. 


\section{Characterization data}<smiles>O=C1c2cccnc2C2Cc3ccccc3N12</smiles>

11,11a-dihydro-5H-pyrido[2',3':3,4]pyrrolo[1,2-a]indol-5-one (2): A $50 \mathrm{~mL}$ Schlenk flask equipped with a stir bar was charged with 3DPAFIPN (26 mg, $2 \mathrm{~mol} \%$ ) and (2-bromopyridin-3yl)(1H-indol-1-yl)methanone (1) (602 mg, $2.0 \mathrm{mmol}, 1$ equiv) and sealed with a septum. The atmosphere was exchanged three times by applying vacuum and backfilling with $\mathrm{N}_{2}$. Under $\mathrm{N}_{2}$ atmosphere, tributylamine $(1.43 \mathrm{~mL}, 6.0 \mathrm{mmol}, 3$ equiv) and degassed $25 \%$ (v/v) toluene/2,2,2trifluoroethanol $(20 \mathrm{~mL}, 0.1 \mathrm{M})$ were added via syringe. The resulting mixture was stirred for 16 hours under irradiation with blue LEDs with an air hose for cooling (see photos 5 and 6 for reaction setup). The reaction was concentrated in vacuo. The crude residue was purified by silica gel chromatography $(50-70 \%$ EtOAc/Hex $+1 \% \mathrm{AcOH})$ to provide the title compound $(325 \mathrm{mg}, 73 \%)$ as an off-white amorphous solid.

${ }^{1}$ H NMR $\left(600 \mathrm{MHz}, \mathrm{CDCl}_{3}\right) \delta 8.78(\mathrm{dd}, J=5.0,1.6 \mathrm{~Hz}, 1 \mathrm{H}), 8.17(\mathrm{dd}, J=7.7,1.6 \mathrm{~Hz}, 1 \mathrm{H}), 7.69$ $(\mathrm{d}, J=7.8 \mathrm{~Hz}, 1 \mathrm{H}), 7.45(\mathrm{dd}, J=7.7,5.0 \mathrm{~Hz}, 1 \mathrm{H}), 7.31(\mathrm{t}, J=7.7 \mathrm{~Hz}, 1 \mathrm{H}), 7.28(\mathrm{~d}, J=7.4 \mathrm{~Hz}$, $1 \mathrm{H}), 7.11(\mathrm{td}, J=7.5,1.1 \mathrm{~Hz}, 1 \mathrm{H}), 5.64(\mathrm{dd}, J=10.4,8.8 \mathrm{~Hz}, 1 \mathrm{H}), 3.56(\mathrm{dd}, J=15.5,9.0 \mathrm{~Hz}$, $1 \mathrm{H}), 3.18(\mathrm{dd}, J=15.5,10.3 \mathrm{~Hz}, 1 \mathrm{H}) \mathrm{ppm}$.

${ }^{13} \mathrm{C}$ NMR $\left(126 \mathrm{MHz}, \mathrm{CDCl}_{3}\right) \delta 166.9,165.9,153.5,140.5,135.9,133.1,128.4,128.3,125.8$, $125.1,123.9,117.0,66.8,32.6 \mathrm{ppm}$.

FTIR (neat) $v_{\max }: 3045,2963,2921,2857,1696,1597,1578,1478,1459,1371,1346,1311,1293$, 1245, 1216, 1109, 1071, 819, 776, 756, 731, 720, 711, 697, 653, 607, 588, 558, 537, $529 \mathrm{~cm}^{-1}$. HRMS (APCI) $m / z$ : $[\mathrm{M}+\mathrm{H}]^{+}$calcd. for $\mathrm{C}_{14} \mathrm{H}_{11} \mathrm{ON} 2$, 223.0866; found, 223.0866.

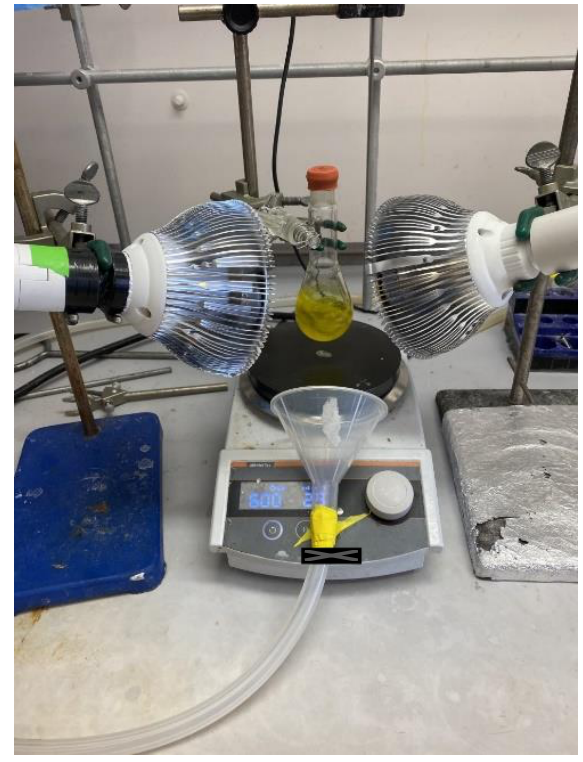

Photo 5

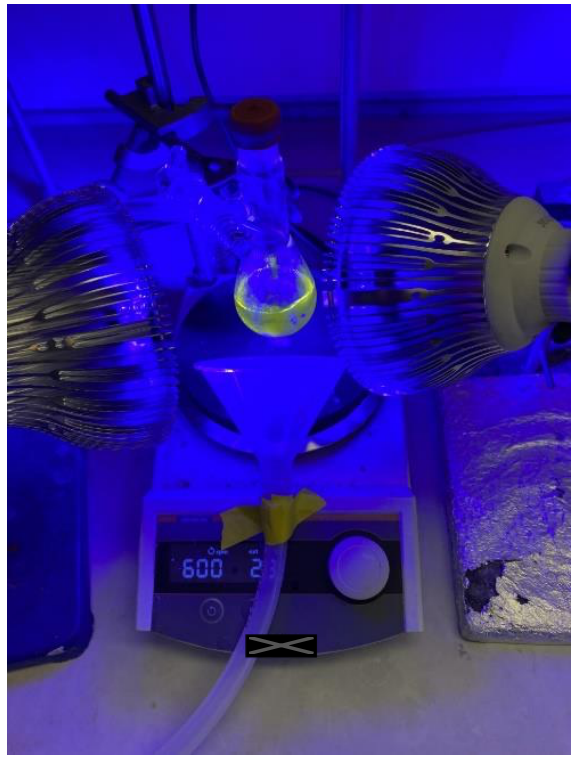

Photo 6 


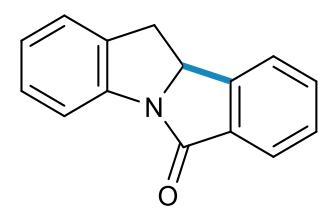

10b,11-dihydro-6H-isoindolo[2,1-a]indol-6-one (3): Following the general hydroarylation procedure B, the reaction of 4CzIPN (20 mg, $5 \mathrm{~mol} \%$ ), (1H-indol-1-yl)(2-iodophenyl)methanone (S2) (104 mg, $0.3 \mathrm{mmol}, 1$ equiv), and $\mathrm{Bu}_{3} \mathrm{~N}$ (0.21 mL, $0.9 \mathrm{mmol}, 3$ equiv) in 2,2,2-trifluoroethanol $(3 \mathrm{~mL}, 0.1 \mathrm{M})$ at $40{ }^{\circ} \mathrm{C}$ provided the title compound $(31.1 \mathrm{mg}, 47 \%)$ as a yellow amorphous solid after purification by silica gel chromatography (5-20\% EtOAc/Hex) and washing with $1 \mathrm{M} \mathrm{HCl}$ (aq).

${ }^{1}$ H NMR $\left(400 \mathrm{MHz}, \mathrm{CDCl}_{3}\right) \delta 7.90(\mathrm{~d}, J=7.5 \mathrm{~Hz}, 1 \mathrm{H}), 7.69(\mathrm{~d}, J=7.9 \mathrm{~Hz}, 1 \mathrm{H}), 7.61(\mathrm{t}, J=7.5$ $\mathrm{Hz}, 1 \mathrm{H}), 7.53-7.48(\mathrm{~m}, 2 \mathrm{H}), 7.29(\mathrm{t}, J=7.7 \mathrm{~Hz}, 1 \mathrm{H}), 7.25(\mathrm{~d}, J=8.1 \mathrm{~Hz}, 1 \mathrm{H}), 7.08(\mathrm{td}, J=7.5$, $1.1 \mathrm{~Hz}, 1 \mathrm{H}), 5.67-5.58(\mathrm{~m}, 1 \mathrm{H}), 3.47(\mathrm{dd}, J=15.3,8.5 \mathrm{~Hz}, 1 \mathrm{H}), 3.06(\mathrm{dd}, J=15.2,10.3 \mathrm{~Hz}$, 1H) $\mathrm{ppm}$.

${ }^{13}$ C NMR $\left(126 \mathrm{MHz}, \mathrm{CDCl}_{3}\right) \delta 168.5,146.2,140.8,136.1,134.4,132.7,128.9,128.1,125.5$, $125.0,124.6,123.0,116.6,65.6,34.0 \mathrm{ppm}$.

FTIR (neat) $v_{\max }: 2957,2927,2871,2858,1696,1478,1461,1372,1302,1160,1139,1100,752$, $729,688,527 \mathrm{~cm}^{-1}$.

HRMS (APCI) $m / z:[\mathrm{M}+\mathrm{H}]^{+}$calcd. for $\mathrm{C}_{15} \mathrm{H}_{12} \mathrm{ON}, 222.0913$; found, 222.0913 .

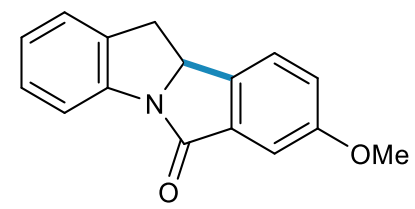

8-methoxy-10b,11-dihydro-6H-isoindolo[2,1-a]indol-6-one (4): Following the general hydroarylation procedure $\mathrm{B}$, the reaction of 4-CzIPN (20 mg, $5 \mathrm{~mol} \%)$, (1H-indol-1-yl)(2-iodo5-methoxyphenyl)methanone (S3) $\left(37.7 \mathrm{mg}, 0.1 \mathrm{mmol}, 1\right.$ equiv), and $\mathrm{Bu}_{3} \mathrm{~N}(71 \mu \mathrm{L}, 0.3 \mathrm{mmol}, 3$ equiv) in 2,2,2-trifluoroethanol $(1 \mathrm{~mL}, 0.1 \mathrm{M})$ at $40{ }^{\circ} \mathrm{C}$ provided the title compound $(15.6 \mathrm{mg}$, $62 \%)$ as a light yellow amorphous solid after purification by silica gel chromatography $(10 \%$ EtOAc/Hex $+1 \% \mathrm{AcOH})$.

${ }^{1}$ H NMR $\left(400 \mathrm{MHz}, \mathrm{CDCl}_{3}\right) \delta 7.67(\mathrm{~d}, J=8.1 \mathrm{~Hz}, 1 \mathrm{H}), 7.40(\mathrm{~d}, J=7.7 \mathrm{~Hz}, 1 \mathrm{H}), 7.36(\mathrm{~d}, J=2.4$ $\mathrm{Hz}, 1 \mathrm{H}), 7.29$ (t, $J=7.7 \mathrm{~Hz}, 1 \mathrm{H}), 7.25-7.22(\mathrm{~m}, 1 \mathrm{H}), 7.16(\mathrm{dd}, J=8.3,2.5 \mathrm{~Hz}, 1 \mathrm{H}), 7.07(\mathrm{td}, J$ $=7.5,1.1 \mathrm{~Hz}, 1 \mathrm{H}), 5.57(\mathrm{dd}, J=10.1,8.6 \mathrm{~Hz}, 1 \mathrm{H}), 3.88(\mathrm{~s}, 3 \mathrm{H}), 3.42(\mathrm{dd}, J=15.2,8.6 \mathrm{~Hz}, 1 \mathrm{H})$, $3.02(\mathrm{dd}, J=15.1,10.2 \mathrm{~Hz}, 1 \mathrm{H}) \mathrm{ppm}$.

${ }^{13} \mathrm{C}$ NMR $\left(126 \mathrm{MHz}, \mathrm{CDCl}_{3}\right) \delta 168.5,160.6,140.9,138.6,136.3,135.8,128.1,125.5,124.6$, $123.8,121.0,116.6,107.6,65.2,55.9,34.2 \mathrm{ppm}$.

FTIR (neat) $v_{\max }: 3069,3043,2919,2860,1688,1680,1600,1469,1451,1432,1357,1277,1225$, $1277,1225,1158,1134,1058,1022,851,828,773,750,734,599,582 \mathrm{~cm}^{-1}$.

HRMS (APCI) $m / z$ : [M+H] $]^{+}$calcd. for $\mathrm{C}_{16} \mathrm{H}_{14} \mathrm{O}_{2} \mathrm{~N}, 252.1019$; found, 252.1016. 


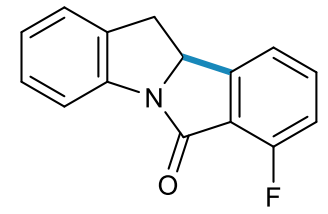

7-fluoro-10b,11-dihydro-6H-isoindolo[2,1-a]indol-6-one (5): Following the general hydroarylation procedure $\mathrm{B}$, the reaction of 4-CzIPN (20 $\mathrm{mg}, 5 \mathrm{~mol} \%$ ), (2-fluoro-6iodophenyl)(1H-imidazol-1-yl)methanone (S4) $\left(110 \mathrm{mg}, 0.3 \mathrm{mmol}, 1\right.$ equiv), and $\mathrm{Bu}_{3} \mathrm{~N}(0.21 \mathrm{~mL}$, $0.9 \mathrm{mmol}, 3$ equiv) in 2,2,2-trifluoroethanol $(3 \mathrm{~mL}, 0.1 \mathrm{M})$ at $40{ }^{\circ} \mathrm{C}$ provided the title compound $(50.2 \mathrm{mg}, 70 \%)$ as a yellow amorphous solid after purification by silica gel chromatography (10$25 \% \mathrm{EtOAc} / \mathrm{Hex})$ and washing with $1 \mathrm{M} \mathrm{HCl}(\mathrm{aq})$.

${ }^{1} \mathbf{H}$ NMR $\left(600 \mathrm{MHz}, \mathrm{CDCl}_{3}\right) \delta 7.67(\mathrm{~d}, J=7.7 \mathrm{~Hz}, 1 \mathrm{H}), 7.58(\mathrm{td}, J=7.9,4.6 \mathrm{~Hz}, 1 \mathrm{H}), 7.29(\mathrm{t}, J$ $=7.7 \mathrm{~Hz}, 2 \mathrm{H}), 7.24(\mathrm{~d}, J=7.3 \mathrm{~Hz}, 1 \mathrm{H}), 7.13(\mathrm{t}, J=9.1 \mathrm{~Hz}, 1 \mathrm{H}), 7.09(\mathrm{t}, J=7.5 \mathrm{~Hz}, 1 \mathrm{H}), 5.60$ (dd, $J=9.9,9.1 \mathrm{~Hz}, 1 \mathrm{H}), 3.46(\mathrm{dd}, J=15.1,8.8 \mathrm{~Hz}, 1 \mathrm{H}), 3.08$ (dd, $J=15.2,10.4 \mathrm{~Hz}, 1 \mathrm{H}) \mathrm{ppm}$. ${ }^{13}$ C NMR $\left(126 \mathrm{MHz}, \mathrm{CDCl}_{3}\right) \delta 165.29\left(\mathrm{~d},{ }^{1} J_{\mathrm{C}-\mathrm{F}}=1.6 \mathrm{~Hz}\right), 159.52\left(\mathrm{~d},{ }^{1} J_{\mathrm{C}-\mathrm{F}}=262.1 \mathrm{~Hz}\right), 148.78(\mathrm{~d}$, $\left.{ }^{3} J_{\mathrm{C}-\mathrm{F}}=2.8 \mathrm{~Hz}\right), 140.59,135.68,134.83\left(\mathrm{~d},{ }^{3} J_{\mathrm{C}-\mathrm{F}}=7.8 \mathrm{~Hz}\right), 128.15,125.44,124.74,121.40\left(\mathrm{~d},{ }^{2} J_{\mathrm{C}-}\right.$ $\mathrm{F}=13.5 \mathrm{~Hz}), 119.01\left(\mathrm{~d},{ }^{4} J_{\mathrm{C}-\mathrm{F}}=4.1 \mathrm{~Hz}\right), 116.74,116.17\left(\mathrm{~d},{ }^{2} J_{\mathrm{C}-\mathrm{F}}=19.3 \mathrm{~Hz}\right), 65.16\left(\mathrm{~d},{ }^{4} J_{\mathrm{C}-\mathrm{F}}=1.4\right.$ $\mathrm{Hz}), 33.95 \mathrm{ppm}$.

${ }^{19} \mathbf{F}$ NMR $\left(376 \mathrm{MHz}, \mathrm{CDCl}_{3}\right) \delta-116.0(\mathrm{dd}, J=9.1,4.8 \mathrm{~Hz}) \mathrm{ppm}$.

FTIR (neat) $v_{\max }: 3055,3024,2908,2853,1692,1626,1590,1476,1461,1369,1308,1293,1251$, $1208,1160,1139,1081,1053,933,796,742,724,705,691,662,635,598 \mathrm{~cm}^{-1}$.

HRMS (APCI) $m / z:[\mathrm{M}+\mathrm{H}]^{+}$calcd. for $\mathrm{C}_{15} \mathrm{H}_{11} \mathrm{ONF}, 240.0819$; found, 240.0820 .

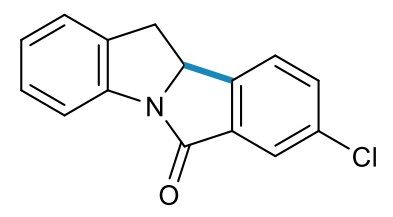

8-chloro-10b,11-dihydro-6H-isoindolo[2,1-a]indol-6-one (6): Following the general hydroarylation procedure $\mathrm{B}$, the reaction of 4-CzIPN (20 mg, $5 \mathrm{~mol} \%)$, (5-chloro-2iodophenyl)(1H-indol-1-yl)methanone (S5) (38.2 mg, $0.1 \mathrm{mmol}, 1$ equiv), and $\mathrm{Bu}_{3} \mathrm{~N}(71 \mu \mathrm{L}, 0.3$ mmol, 3 equiv) in 2,2,2-trifluoroethanol $(1 \mathrm{~mL}, 0.1 \mathrm{M})$ at $40{ }^{\circ} \mathrm{C}$ provided the title compound (17.0 $\mathrm{mg}, 66 \%)$ as a yellow amorphous solid after purification by silica gel chromatography (5-15\% EtOAc/Hex) and washing with $1 \mathrm{M} \mathrm{HCl}(\mathrm{aq})$.

${ }^{1} \mathbf{H}$ NMR $\left(600 \mathrm{MHz}, \mathrm{CDCl}_{3}\right) \delta 7.86(\mathrm{~d}, J=1.9 \mathrm{~Hz}, 1 \mathrm{H}), 7.67(\mathrm{~d}, J=7.7 \mathrm{~Hz}, 1 \mathrm{H}), 7.57(\mathrm{dd}, J=$ 8.0, $2.0 \mathrm{~Hz}, 1 \mathrm{H}), 7.45(\mathrm{dd}, J=8.2,0.7 \mathrm{~Hz}, 1 \mathrm{H}), 7.30(\mathrm{t}, J=7.7 \mathrm{~Hz}, 1 \mathrm{H}), 7.25(\mathrm{~d}, J=7.6 \mathrm{~Hz}, 0 \mathrm{H})$, 7.09 (t, $J=7.5 \mathrm{~Hz}, 1 \mathrm{H}), 5.59$ (dd, $J=10.4,8.6 \mathrm{~Hz}, 1 \mathrm{H}), 3.46$ (dd, $J=15.1,8.6 \mathrm{~Hz}, 1 \mathrm{H}), 3.05$ (dd, $J=15.0,10.3 \mathrm{~Hz}, 1 \mathrm{H}) \mathrm{ppm}$.

${ }^{13}$ C NMR $\left(126 \mathrm{MHz}, \mathrm{CDCl}_{3}\right) \delta 166.9,144.2,140.5,136.2,135.9,135.2,132.8,128.2,125.6$, $125.0,124.9,124.2,116.6,65.3,33.9 \mathrm{ppm}$.

FTIR (neat) $v_{\max }: 3079,3037,2963,2908,2847,1697,1603,1477,1460,1417,1365,1308,1290$, $1259,1207,1194,1175,1134,1116,1078,1050,889,830,775,749,719,690,667,594 \mathrm{~cm}^{-1}$.

HRMS (APCI) $m / z$ : [M+H] $]^{+}$calcd. for $\mathrm{C}_{15} \mathrm{H}_{11} \mathrm{ONCl}$, 256.0524; found, 256.0524. 
<smiles>Cc1ccc2c(c1)N1C(=O)c3cccnc3C1C2</smiles>

8-methyl-11,11a-dihydro-5H-pyrido[2',3':3,4]pyrrolo[1,2-a]indol-5-one (7): Following the general hydroarylation procedure $A$, the reaction of 3DPAFIPN (16 mg, $5 \mathrm{~mol} \%$ ), (2bromopyridin-3-yl)(6-methyl-1H-indol-1-yl)methanone (S6) (158 mg, $0.5 \mathrm{mmol}, 1 \mathrm{equiv})$, and $\mathrm{Bu}_{3} \mathrm{~N}(0.36 \mathrm{~mL}, 1.5 \mathrm{mmol}, 3$ equiv) provided the title compound $(100 \mathrm{mg}, 85 \%)$ as an off-white amorphous solid after purification by silica gel chromatography $(50 \%$ EtOAc/Hex $+1 \% \mathrm{AcOH})$.

${ }^{1} \mathbf{H}$ NMR $\left(500 \mathrm{MHz}, \mathrm{CDCl}_{3}\right) \delta 8.78(\mathrm{dd}, J=4.9,1.5 \mathrm{~Hz}, 1 \mathrm{H}), 8.17(\mathrm{dd}, J=7.7,1.2 \mathrm{~Hz}, 1 \mathrm{H}), 7.52$ (s, 1H), $7.44(\mathrm{ddd}, J=7.7,4.9,0.6 \mathrm{~Hz}, 1 \mathrm{H}), 7.15(\mathrm{~d}, J=7.6 \mathrm{~Hz}, 1 \mathrm{H}), 6.92(\mathrm{~d}, J=7.6 \mathrm{~Hz}, 1 \mathrm{H})$, $5.62(\mathrm{dd}, J=10.2,9.0 \mathrm{~Hz}, 1 \mathrm{H}), 3.51$ (dd, $J=15.3,8.9 \mathrm{~Hz}, 1 \mathrm{H}), 3.13(\mathrm{dd}, J=15.8,10.5 \mathrm{~Hz}, 1 \mathrm{H})$, $2.41(\mathrm{~s}, 3 \mathrm{H}) \mathrm{ppm}$.

${ }^{13} \mathrm{C}$ NMR $\left(126 \mathrm{MHz}, \mathrm{CDCl}_{3}\right) \delta 166.6,165.8,153.3,140.4,138.2,132.9,132.8,128.3,125.6$, $125.2,123.7,117.5,67.0,32.1,21.5 \mathrm{ppm}$.

FTIR (neat) $v_{\max }: 2955,2923,2854,1693,1602,1580,1493,1418,1361,1303,1193,1090,862$, $810,782,760,727,697,657,592,555,543 \mathrm{~cm}^{-1}$.

HRMS (APCI) $m / z$ : $[\mathrm{M}+\mathrm{H}]^{+}$calcd. for $\mathrm{C}_{15} \mathrm{H}_{13} \mathrm{ON}_{2}, 237.1022$; found, 237.1020.<smiles>CCCCOc1cccc2c1CC1c3ncccc3C(=O)N21</smiles>

10-((tert-butyldimethylsilyl)oxy)-11,11a-dihydro-5H-pyrido[2',3':3,4]pyrrolo[1,2-a]indol-5one (8): Following the general hydroarylation procedure A, the reaction of 3DPAFIPN (16 mg, 5 mol \%), (2-bromopyridin-3-yl)(4-((tert-butyldimethylsilyl)oxy)-1H-indol-1-yl)methanone (S7) ( $216 \mathrm{mg}, 0.5 \mathrm{mmol}, 1$ equiv), and $\mathrm{Bu}_{3} \mathrm{~N}(0.36 \mathrm{~mL}, 1.5 \mathrm{mmol}, 3$ equiv) provided the title compound (151 mg, 86\%) as an off-white amorphous solid after purification by silica gel chromatography $(20-30 \% \mathrm{EtOAc} / \mathrm{Hex}+1 \% \mathrm{AcOH})$.

${ }^{1} \mathbf{H}$ NMR $\left(400 \mathrm{MHz}, \mathrm{CDCl}_{3}\right) \delta 8.78(\mathrm{dd}, J=5.0,1.6 \mathrm{~Hz}, 1 \mathrm{H}), 8.16(\mathrm{dd}, J=7.8,1.6 \mathrm{~Hz}, 1 \mathrm{H}), 7.45$ $(\mathrm{dd}, J=7.7,5.0 \mathrm{~Hz}, 1 \mathrm{H}), 7.32(\mathrm{~d}, J=7.3 \mathrm{~Hz}, 1 \mathrm{H}), 7.18(\mathrm{t}, J=8.0 \mathrm{~Hz}, 1 \mathrm{H}), 6.58(\mathrm{~d}, J=8.1 \mathrm{~Hz}$, $1 \mathrm{H}), 5.62(\mathrm{t}, J=9.6 \mathrm{~Hz}, 1 \mathrm{H}), 3.57(\mathrm{dd}, J=15.6,9.1 \mathrm{~Hz}, 1 \mathrm{H}), 3.03(\mathrm{dd}, J=15.4,10.0 \mathrm{~Hz}, 1 \mathrm{H})$, $0.99(\mathrm{~s}, 9 \mathrm{H}), 0.24(\mathrm{~s}, 3 \mathrm{H}), 0.17(\mathrm{~s}, 3 \mathrm{H}) \mathrm{ppm}$.

${ }^{13}$ C NMR $\left(126 \mathrm{MHz}, \mathrm{CDCl}_{3}\right) \delta 166.0,153.4,152.6,141.9,133.1,129.3,128.4,125.8,123.9$, $116.1,110.2,66.9,29.9,25.8,18.3,-4.0,-4.1 \mathrm{ppm}$.

FTIR (neat) vmax: 2949, 2928, 2901, 2858, 1691, 1591, 1472, 1453, 1371, 1307, 1286, 1248, 1205 , $1141,1108,1001,862,837,823,811,797,772,707,667,603,571 \mathrm{~cm}^{-1}$.

HRMS (APCI) $m / z$ : [M+H] $]^{+}$calcd. for $\mathrm{C}_{20} \mathrm{H}_{25} \mathrm{O}_{2} \mathrm{~N}_{2} \mathrm{Si}$, 353.1680; found, 353.1673 . 


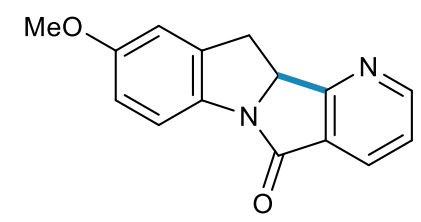

9-methoxy-11,11a-dihydro-5H-pyrido[2',3':3,4]pyrrolo[1,2-a]indol-5-one (9): Following the general hydroarylation procedure $\mathrm{A}$, the reaction of 3DPAFIPN (16 $\mathrm{mg}, 5 \mathrm{~mol} \%$ ), (2bromopyridin-3-yl)(5-methoxy-1H-indol-1-yl)methanone (S8) (166 mg, $0.5 \mathrm{mmol}, 1$ equiv), and $\mathrm{Bu}_{3} \mathrm{~N}(0.36 \mathrm{~mL}, 1.5 \mathrm{mmol}, 3$ equiv) provided the title compound (105 $\mathrm{mg}, 83 \%)$ as a light yellow amorphous solid after purification by silica gel chromatography $(50-70 \%$ EtOAc/Hex $+1 \%$ $\mathrm{AcOH})$.

${ }^{1} \mathbf{H}$ NMR $\left(600 \mathrm{MHz}, \mathrm{CDCl}_{3}\right) \delta 8.76(\mathrm{dd}, J=5.0,1.4 \mathrm{~Hz}, 1 \mathrm{H}), 8.15(\mathrm{dd}, J=7.8,0.9 \mathrm{~Hz}, 1 \mathrm{H}), 7.58$ $(\mathrm{d}, J=8.5 \mathrm{~Hz}, 1 \mathrm{H}), 7.44(\mathrm{dd}, J=7.7,4.9 \mathrm{~Hz}, 1 \mathrm{H}), 6.85(\mathrm{~s}, 1 \mathrm{H}), 6.82(\mathrm{dd}, J=8.5,2.6 \mathrm{~Hz}, 1 \mathrm{H})$, $5.65-5.58(\mathrm{~m}, 1 \mathrm{H}), 3.80(\mathrm{~s}, 3 \mathrm{H}), 3.52(\mathrm{dd}, J=15.5,8.8 \mathrm{~Hz}, 1 \mathrm{H}), 3.15(\mathrm{dd}, J=15.5,10.3 \mathrm{~Hz}$, 1H) ppm.

${ }^{13}$ C NMR $\left(126 \mathrm{MHz} \mathrm{CDCl}_{3}\right) \delta 166.7,165.8,157.5,153.2,137.5,134.0,132.9,128.4,123.8$, $117.3,112.7,112.3,67.1,55.9,32.8 \mathrm{ppm}$.

FTIR (neat) $v_{\max }: 3069,2961,2929,2837,1692,1581,1488,1476,1440,1381,1315,1239,1230$, 1139, 1094, 1072, 1021, 859, 807, 773, 773, 749, 728, 717, 549, $530 \mathrm{~cm}^{-1}$.

HRMS (APCI) $m / z$ : $[\mathrm{M}+\mathrm{H}]^{+}$calcd. for $\mathrm{C}_{15} \mathrm{H}_{13} \mathrm{O}_{2} \mathrm{~N}_{2}, 253.0972$; found, 253.0972.<smiles>COc1ccc2c(c1)N1C(=O)c3cccnc3C1C2</smiles>

8-methoxy-11,11a-dihydro-5H-pyrido[2',3':3,4]pyrrolo[1,2-a]indol-5-one (10): Following the general hydroarylation procedure $A$, the reaction of 3DPAFIPN (16 mg, $5 \mathrm{~mol} \%$ ), (2bromopyridin-3-yl)(6-methoxy-1H-indol-1-yl)methanone (S9) (166 mg, $0.5 \mathrm{mmol}, 1$ equiv), and $\mathrm{Bu}_{3} \mathrm{~N}(0.36 \mathrm{~mL}, 1.5 \mathrm{mmol}, 3$ equiv) provided the title compound $(96.3 \mathrm{mg}, 76 \%)$ as a light yellow amorphous solid after purification by silica gel chromatography (40-60\% EtOAc/Hex $+1 \%$ $\mathrm{AcOH})$.

${ }^{1}$ H NMR $\left(400 \mathrm{MHz}, \mathrm{CDCl}_{3}\right) \delta 8.78(\mathrm{dd}, J=4.9,1.6 \mathrm{~Hz}, 1 \mathrm{H}), 8.17(\mathrm{dd}, J=7.7,1.7 \mathrm{~Hz}, 1 \mathrm{H}), 7.45$ $(\mathrm{ddd}, J=7.8,5.0,0.6 \mathrm{~Hz}, 1 \mathrm{H}), 7.29(\mathrm{~d}, J=2.5 \mathrm{~Hz}, 1 \mathrm{H}), 7.15(\mathrm{~d}, J=7.9 \mathrm{~Hz}, 1 \mathrm{H}), 6.65(\mathrm{dd}, J=$ $8.3,2.5 \mathrm{~Hz}, 1 \mathrm{H}), 5.70-5.61(\mathrm{~m}, 1 \mathrm{H}), 3.87(\mathrm{~s}, 3 \mathrm{H}), 3.49(\mathrm{dd}, J=15.0,9.0 \mathrm{~Hz}, 1 \mathrm{H}), 3.11(\mathrm{dd}, J=$ $15.3,10.4 \mathrm{~Hz}, 1 \mathrm{H}) \mathrm{ppm}$.

${ }^{13} \mathrm{C}$ NMR $\left(126 \mathrm{MHz}, \mathrm{CDCl}_{3}\right) \delta 166.7,166.0,160.1,153.5,141.5,133.1,128.3,127.4,126.0$, $123.9,111.0,103.1,67.5,55.9,31.8 \mathrm{ppm}$.

FTIR (neat) $v_{\max }: 3079,2996,2917,2832,1694,1581,1491,1452,1438,1366,1310,1282,1213$, $1166,1143,1093,1027,851,795,780,756,727,696,655,619,578,555,533 \mathrm{~cm}^{-1}$.

HRMS (APCI) $m / z:[\mathrm{M}+\mathrm{H}]^{+}$calcd. for $\mathrm{C}_{15} \mathrm{H}_{13} \mathrm{O}_{2} \mathrm{~N}_{2}$, 253.0972; found, 253.0971. 
<smiles>O=C1c2cccnc2C2Cc3c(Br)cccc3N12</smiles>

10-bromo-11,11a-dihydro-5H-pyrido $\left[2^{\prime}, 3^{\prime}: 3,4\right]$ pyrrolo[1,2-a]indol-5-one (11): Following the general hydroarylation procedure $\mathrm{A}$, the reaction of 3DPAFIPN (16 mg, $5 \mathrm{~mol} \%)$, (4-bromo-1Hindol-1-yl)(2-bromopyridin-3-yl)methanone (S10) (190 mg, $0.5 \mathrm{mmol}, 1$ equiv), and $\mathrm{Bu}_{3} \mathrm{~N}(0.36$ $\mathrm{mL}, 1.5 \mathrm{mmol}, 3$ equiv) provided the title compound $(125 \mathrm{mg}, 83 \%)$ as an off-white amorphous solid after purification by silica gel chromatography (30-50\% EtOAc/Hex $+1 \% \mathrm{AcOH})$.

${ }^{1} \mathbf{H}$ NMR $\left(400 \mathrm{MHz}, \mathrm{CDCl}_{3}\right) \delta 8.81(\mathrm{dd}, J=4.9,1.6 \mathrm{~Hz}, 1 \mathrm{H}), 8.17(\mathrm{dd}, J=7.7,1.7 \mathrm{~Hz}, 1 \mathrm{H}), 7.62$ $(\mathrm{dd}, J=7.6,1.1 \mathrm{~Hz}, 1 \mathrm{H}), 7.47(\mathrm{ddd}, J=7.7,4.9,0.6 \mathrm{~Hz}, 1 \mathrm{H}), 7.29-7.22(\mathrm{~m}, 1 \mathrm{H}), 7.23-7.14$ $(\mathrm{m}, 1 \mathrm{H}), 5.66(\mathrm{t}, J=9.6 \mathrm{~Hz}, 1 \mathrm{H}), 3.63(\mathrm{dd}, J=16.2,9.2 \mathrm{~Hz}, 1 \mathrm{H}), 3.18(\mathrm{dd}, J=16.6,10.2 \mathrm{~Hz}$, 1H) ppm.

${ }^{13}$ C NMR $\left(126 \mathrm{MHz}, \mathrm{CDCl}_{3}\right) \delta$ 167.2, 165.8, 153.8, 141.6, 136.4, 133.2, 129.8, 128.0, 127.8, 124.0, 119.8, 115.6, 65.7, $33.9 \mathrm{ppm}$.

FTIR (neat) $v_{\max }: 2961,2927,2860,1712,1596,1449,1417,1356,1301,1267,1230,1157,1100$, $1071,891,870,827,811,773,736,694,678,600,571,542 \mathrm{~cm}^{-1}$.

HRMS (APCI) $m / z$ : $[\mathrm{M}+\mathrm{H}]^{+}$calcd. for $\mathrm{C}_{14} \mathrm{H}_{10} \mathrm{ON}{ }_{2} \mathrm{Br}, 300.9971$; found, 300.9970 .<smiles>O=C1c2cccnc2C2Cc3cc(Br)ccc3N12</smiles>

9-bromo-11,11a-dihydro-5H-pyrido[2',3':3,4]pyrrolo[1,2-a]indol-5-one (12): Following the general hydroarylation procedure A, the reaction of 3DPAFIPN (16 mg, $5 \mathrm{~mol} \%)$, (5-bromo-1Hindol-1-yl)(2-bromopyridin-3-yl)methanone (S11) (190 mg, $0.5 \mathrm{mmol}, 1$ equiv), and $\mathrm{Bu}_{3} \mathrm{~N}(0.36$ $\mathrm{mL}, 1.5 \mathrm{mmol}, 3$ equiv) provided the title compound $(110 \mathrm{mg}, 73 \%)$ as an off-white amorphous solid after purification by silica gel chromatography $(30 \%$ EtOAc/Hex $+1 \% \mathrm{AcOH})$.

${ }^{1} \mathbf{H}$ NMR $\left(600 \mathrm{MHz}, \mathrm{CDCl}_{3}\right){ }^{1} \mathrm{H}$ NMR $(600 \mathrm{MHz}$, Chloroform- $d$ ) $\delta 8.79$ (ddd, $J=4.9,1.6,0.6$ $\mathrm{Hz}, 1 \mathrm{H}), 8.17(\mathrm{dd}, J=7.8,1.6 \mathrm{~Hz}, 1 \mathrm{H}), 7.55(\mathrm{~d}, J=8.1 \mathrm{~Hz}, 1 \mathrm{H}), 7.46$ (ddt, $J=7.7,4.9,0.7 \mathrm{~Hz}$, $1 \mathrm{H}), 7.44-7.41(\mathrm{~m}, 2 \mathrm{H}), 5.66-5.62(\mathrm{~m}, 1 \mathrm{H}), 3.55(\mathrm{dd}, J=15.7,9.0 \mathrm{~Hz}, 1 \mathrm{H}), 3.18(\mathrm{dd}, J=$ $15.8,10.3 \mathrm{~Hz}, 1 \mathrm{H}) \mathrm{ppm}$.

${ }^{13}$ C NMR $\left(126 \mathrm{MHz}, \mathrm{CDCl}_{3}\right) \delta 166.8,165.6,153.7,139.6,138.0,133.2,131.1,128.8,127.9$, 124.0, 118.0, 117.8, 66.6, 32.4 ppm.

FTIR (neat) $v_{\max }: 2956,2927,2870,2797,1694,1599,1582,1467,1364,1300,1177,826,771$, $734,710,533 \mathrm{~cm}^{-1}$.

HRMS (APCI) $m / z$ : $[\mathrm{M}+\mathrm{H}]^{+}$calcd. For $\mathrm{C}_{14} \mathrm{H}_{10} \mathrm{ON}_{2} \mathrm{Br}, 300.9971$; found, 300.9974 . 
<smiles>O=C1c2cccnc2C2Cc3ccc(Br)cc3N12</smiles>

8-bromo-11,11a-dihydro-5H-pyrido[2',3':3,4]pyrrolo[1,2-a]indol-5-one (13): Following the general hydroarylation procedure $\mathrm{A}$, the reaction of 3DPAFIPN $(16 \mathrm{mg}, 5 \mathrm{~mol} \%)$, (6-bromo-1Hindol-1-yl)(2-bromopyridin-3-yl)methanone (S12) (190 mg, $0.5 \mathrm{mmol}, 1$ equiv), and $\mathrm{Bu}_{3} \mathrm{~N}(0.36$ $\mathrm{mL}, 1.5 \mathrm{mmol}, 3$ equiv) provided the title compound (105 $\mathrm{mg}, 69 \%)$ as an off-white amorphous solid after purification by silica gel chromatography (30-50\% EtOAc/Hex $+1 \% \mathrm{AcOH})$.

${ }^{1}$ H NMR $\left(600 \mathrm{MHz}, \mathrm{CDCl}_{3}\right) \delta 8.80(\mathrm{dd}, J=4.9,1.6 \mathrm{~Hz}, 1 \mathrm{H}), 8.18(\mathrm{dd}, J=7.7,1.6 \mathrm{~Hz}, 1 \mathrm{H}), 7.84$ $(\mathrm{d}, J=1.9 \mathrm{~Hz}, 1 \mathrm{H}), 7.46(\mathrm{ddd}, J=7.7,5.0,0.6 \mathrm{~Hz}, 1 \mathrm{H}), 7.24(\mathrm{dd}, J=8.0,1.9 \mathrm{~Hz}, 1 \mathrm{H}), 7.13(\mathrm{dt}, J$ $=8.0,1.1 \mathrm{~Hz}, 1 \mathrm{H}), 5.68-5.62(\mathrm{~m}, 1 \mathrm{H}), 3.52(\mathrm{dd}, J=15.6,9.0 \mathrm{~Hz}, 1 \mathrm{H}), 3.12(\mathrm{ddd}, J=15.7$, $10.3,1.4 \mathrm{~Hz}, 1 \mathrm{H}) \mathrm{ppm}$.

${ }^{13}$ C NMR (126 MHz, $\left.\mathrm{CDCl}_{3}\right) \delta 166.8,165.7,153.7,141.7,134.8,133.2,127.9,127.8,126.8$, 124.0, 121.4, 120.0, 66.9, $32.2 \mathrm{ppm}$.

FTIR (neat) $v_{\max }: 2956,2927,2876,2806,1695,1593,1581,1473,1417,1358,1298,1115,1070$, $860,806,774,736,708,646,584,550 \mathrm{~cm}^{-1}$.

HRMS (APCI) $m / z$ : $[\mathrm{M}+\mathrm{H}]^{+}$calcd. for $\mathrm{C}_{14} \mathrm{H}_{10} \mathrm{ON}{ }_{2} \mathrm{Br}, 300.9971$; found, 300.9973 .<smiles>C1=CC2(C=CC1)C=COc1cccnc12</smiles>

2' $H$-spiro[cyclohexane-1,3'-furo[3,2-b]pyridine]-2,5-diene (21): Following the general hydroarylation procedure $\mathrm{A}$, the reaction of 3DPAFIPN (16 mg, $5 \mathrm{~mol} \%$ ), 3-(benzyloxy)-2bromopyridine (17) (132 mg, $0.5 \mathrm{mmol}, 1$ equiv) and $\mathrm{Bu}_{3} \mathrm{~N}(0.28 \mathrm{~mL}, 1.5 \mathrm{mmol}, 3$ equiv) provided the title compound $(24.5 \mathrm{mg}, 26 \%)$ as an off-white amorphous solid after purification by silica gel chromatography $(20-60 \% \mathrm{EtOAc} / \mathrm{Hex}+1 \% \mathrm{AcOH})$.

${ }^{1}$ H NMR $\left(600 \mathrm{MHz}_{\mathrm{CDCl}}\right) \delta 8.14(\mathrm{dd}, J=4.7,1.4 \mathrm{~Hz}, 1 \mathrm{H}), 7.09(\mathrm{dd}, J=8.1,1.4 \mathrm{~Hz}, 1 \mathrm{H}), 7.05$ $(\mathrm{dd}, J=8.1,4.7 \mathrm{~Hz}, 1 \mathrm{H}), 6.02(\mathrm{dt}, J=10.2,3.4 \mathrm{~Hz}, 2 \mathrm{H}), 5.75(\mathrm{dt}, J=10.3,2.0 \mathrm{~Hz}, 2 \mathrm{H}), 4.46$ (s, 2H), 2.93 (dtt, $J=23.3,3.3,2.1 \mathrm{~Hz}, 1 \mathrm{H}), 2.74$ (dtt, $J=23.4,3.5,2.1 \mathrm{~Hz}, 1 \mathrm{H}) \mathrm{ppm}$.

${ }^{1} \mathrm{H}$ NMR spectrum is consistent with reported values. ${ }^{11}$

${ }^{11}$ Flynn, A. R.; McDaniel, K. A.; Hughes, M. E.; Vogt, D. B.; Jui, N. T. J. Am. Chem. Soc. 2020, 142, 9163-9168. 


\section{Deuterium Labeling Study}

\section{A. Deuterated amine}

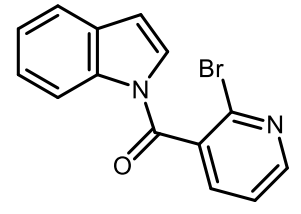

1

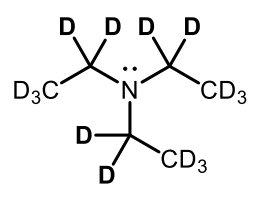

(3 equiv)

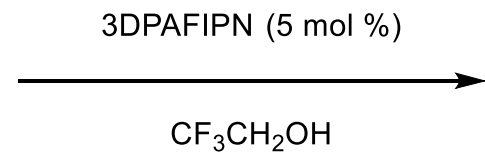

blue LEDs, $23^{\circ} \mathrm{C}, 16 \mathrm{~h}$<smiles>O=C1c2cccnc2C2C([18OH])c3ccccc3N12</smiles>

2: $5 \%$ D

An oven-dried screw-top test tube equipped with a stir bar was charged with 3DPAFIPN $(3.2 \mathrm{mg}$, $5 \mathrm{~mol} \%$ ) and (2-bromopyridin-3-yl)(1H-indol-1-yl)methanone (1) $(30.1 \mathrm{mg}, 0.1 \mathrm{mmol}, 1$ equiv). The atmosphere was exchanged three times by applying vacuum and backfilling with $\mathrm{N}_{2}$. Under $\mathrm{N}_{2}$ atmosphere, triethyl- $\mathrm{d}_{15}$-amine $(42 \mu \mathrm{L}, 0.3 \mathrm{mmol}, 3$ equiv) was added via microsyringe. Degassed 2,2,2-trifluoroethanol $(1 \mathrm{~mL}, 0.1 \mathrm{M})$ was added via syringe and the resulting mixture was stirred for 16 hours under irradiation with blue LEDs. The reaction was concentrated in vacuo, and the crude mixture was analyzed by ${ }^{1} \mathrm{H}$ NMR before purification. The crude residue was purified by silica gel chromatography $(30-50 \% \mathrm{EtOAc} / \mathrm{Hex})$ to provide the desired product. ${ }^{1} \mathrm{H}$ NMR analysis $(\mathrm{d} 1=10 \mathrm{sec})$ showed 5\% deuterium incorporation.

\section{B. Deuterated solvent}

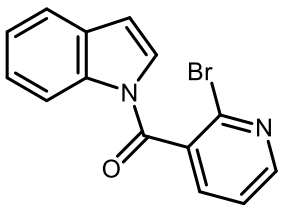

1

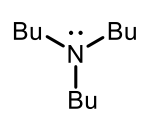

(3 equiv)
3DPAFIPN ( $5 \mathrm{~mol} \%$ )

$\mathrm{CF}_{3} \mathrm{CH}_{2} \mathrm{OD}$

blue LEDs, $23^{\circ} \mathrm{C}, 16 \mathrm{~h}$<smiles>O=C1c2cccnc2C2C([18OH])c3ccccc3N12</smiles>

2: $86 \%$ D

An oven-dried screw-top test tube equipped with a stir bar was charged with 3DPAFIPN (3.2 mg, $5 \mathrm{~mol} \%$ ) and (2-bromopyridin-3-yl)(1H-indol-1-yl)methanone (1) (30.1 mg, $0.1 \mathrm{mmol}, 1$ equiv). The atmosphere was exchanged three times by applying vacuum and backfilling with $\mathrm{N}_{2}$. Under $\mathrm{N}_{2}$ atmosphere, tributylamine ( $71 \mu \mathrm{L}, 0.3 \mathrm{mmol}, 3$ equiv) was added via microsyringe. Degassed $\mathrm{CF}_{3} \mathrm{CH}_{2} \mathrm{OD}(1 \mathrm{~mL}, 0.1 \mathrm{M})$ were added via syringe and the resulting mixture was stirred for 16 hours under irradiation with blue LEDs. The reaction was concentrated in vacuo, and the crude mixture was analyzed by ${ }^{1} \mathrm{H}$ NMR before purification. The crude residue was purified by silica gel chromatography $\left(30-50 \%\right.$ EtOAc/Hex) to provide the desired product. ${ }^{1} \mathrm{H}$ NMR analysis $(\mathrm{d} 1=10$ sec) showed $86 \%$ deuterium incorporation. 


\section{NMR Spectra}

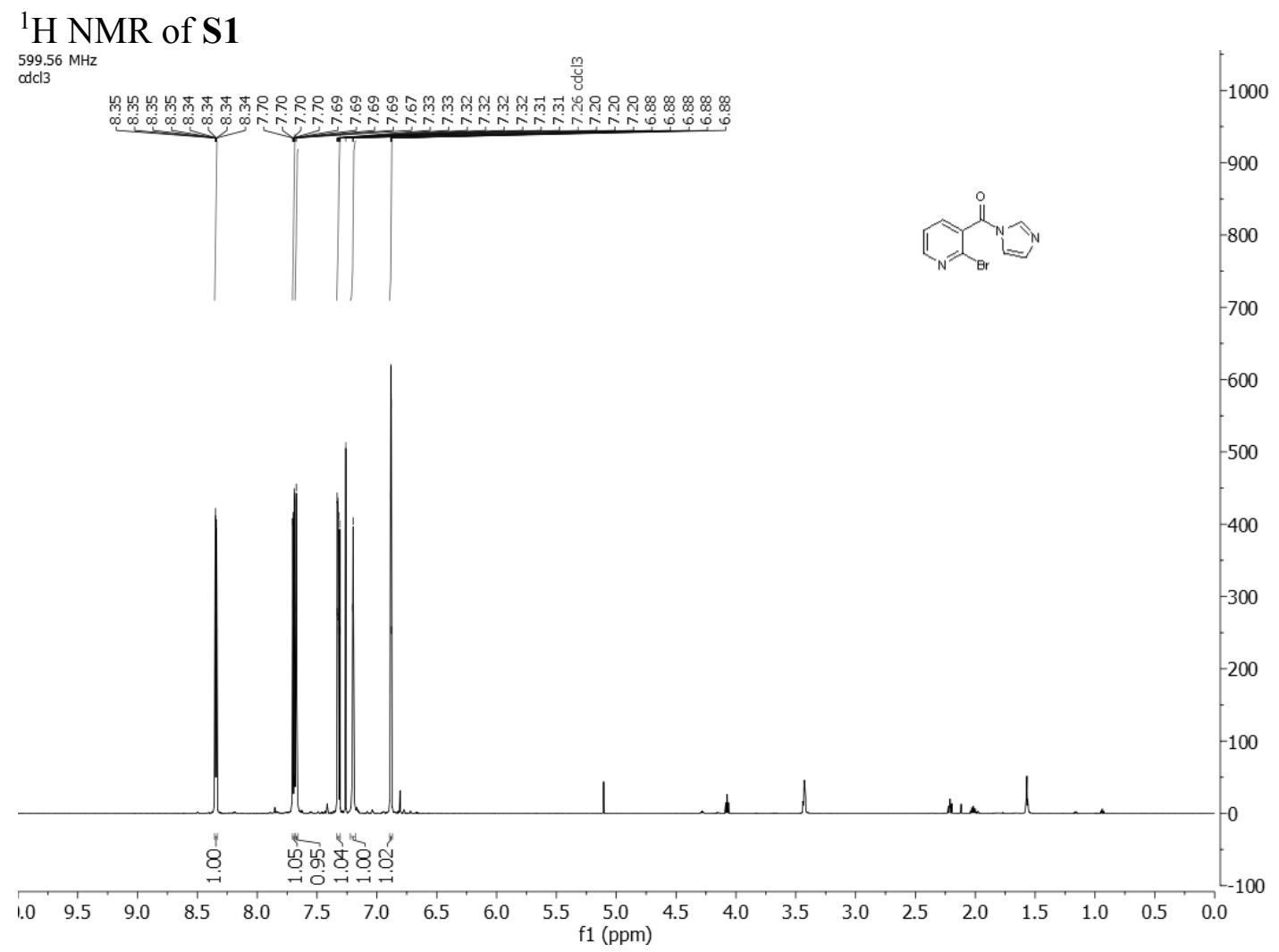

${ }^{13} \mathrm{C}$ NMR of $\mathbf{S 1}$

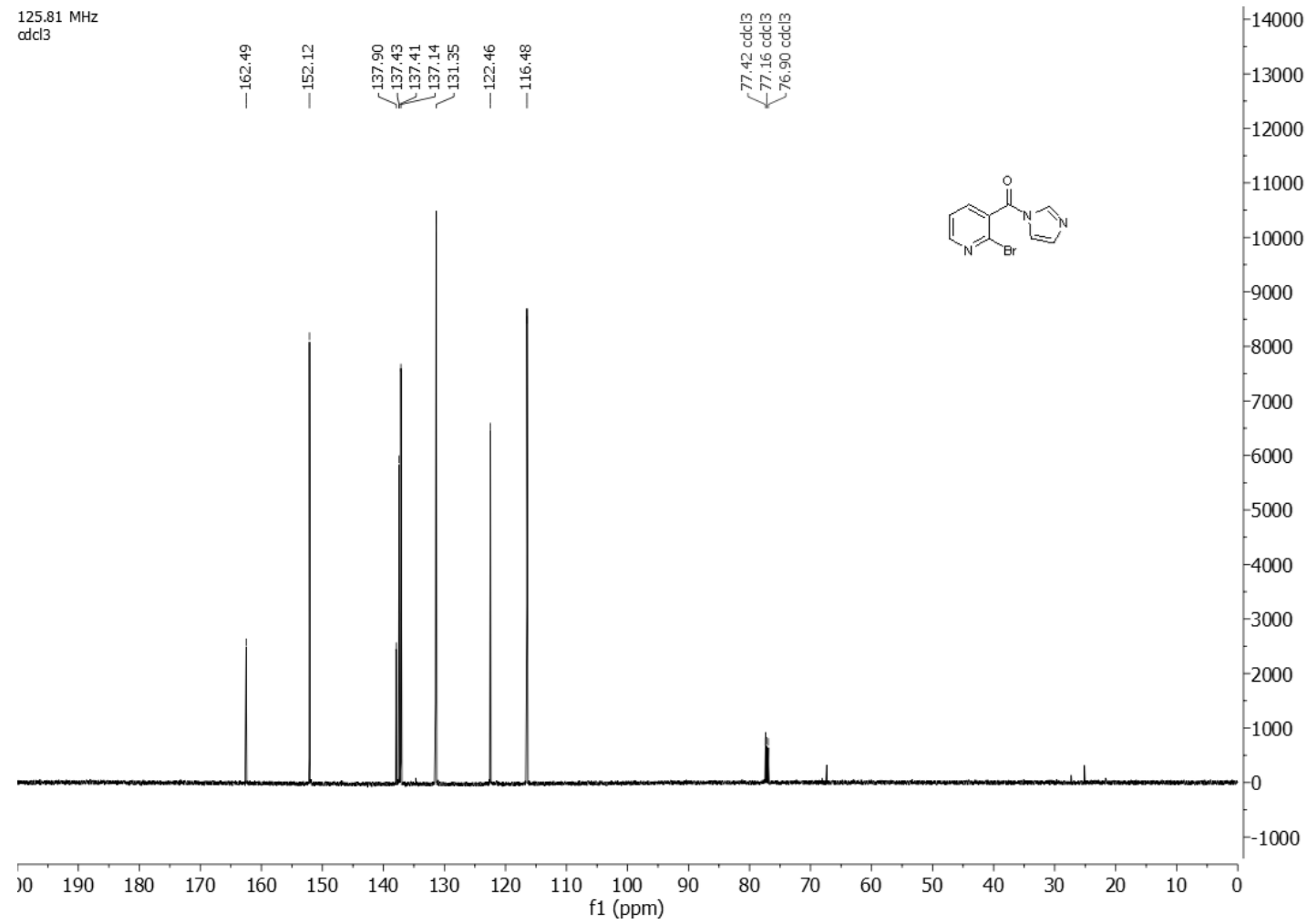




\section{${ }^{1} \mathrm{H}$ NMR of 1}

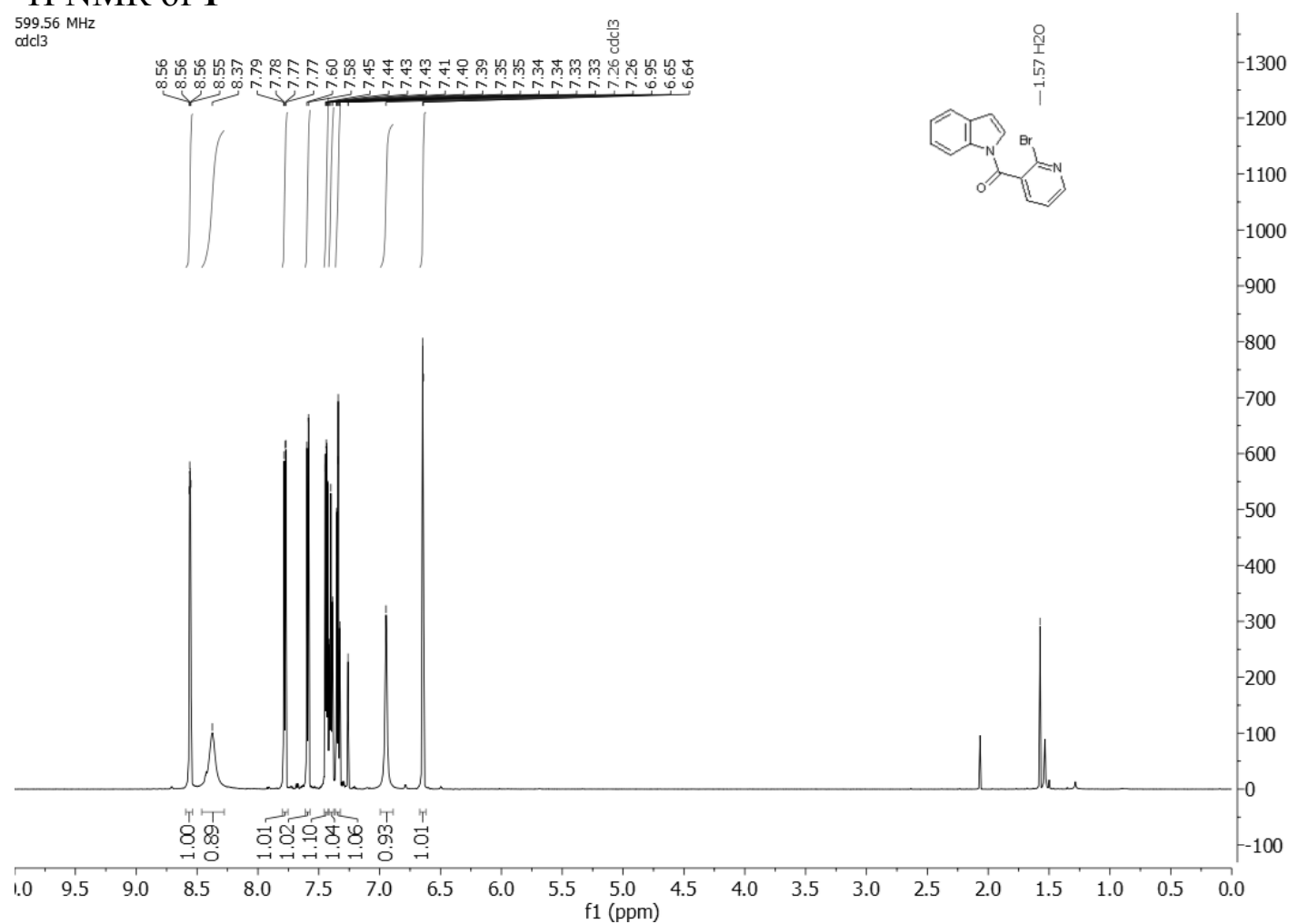

${ }^{13} \mathrm{C}$ NMR of $\mathbf{1}$

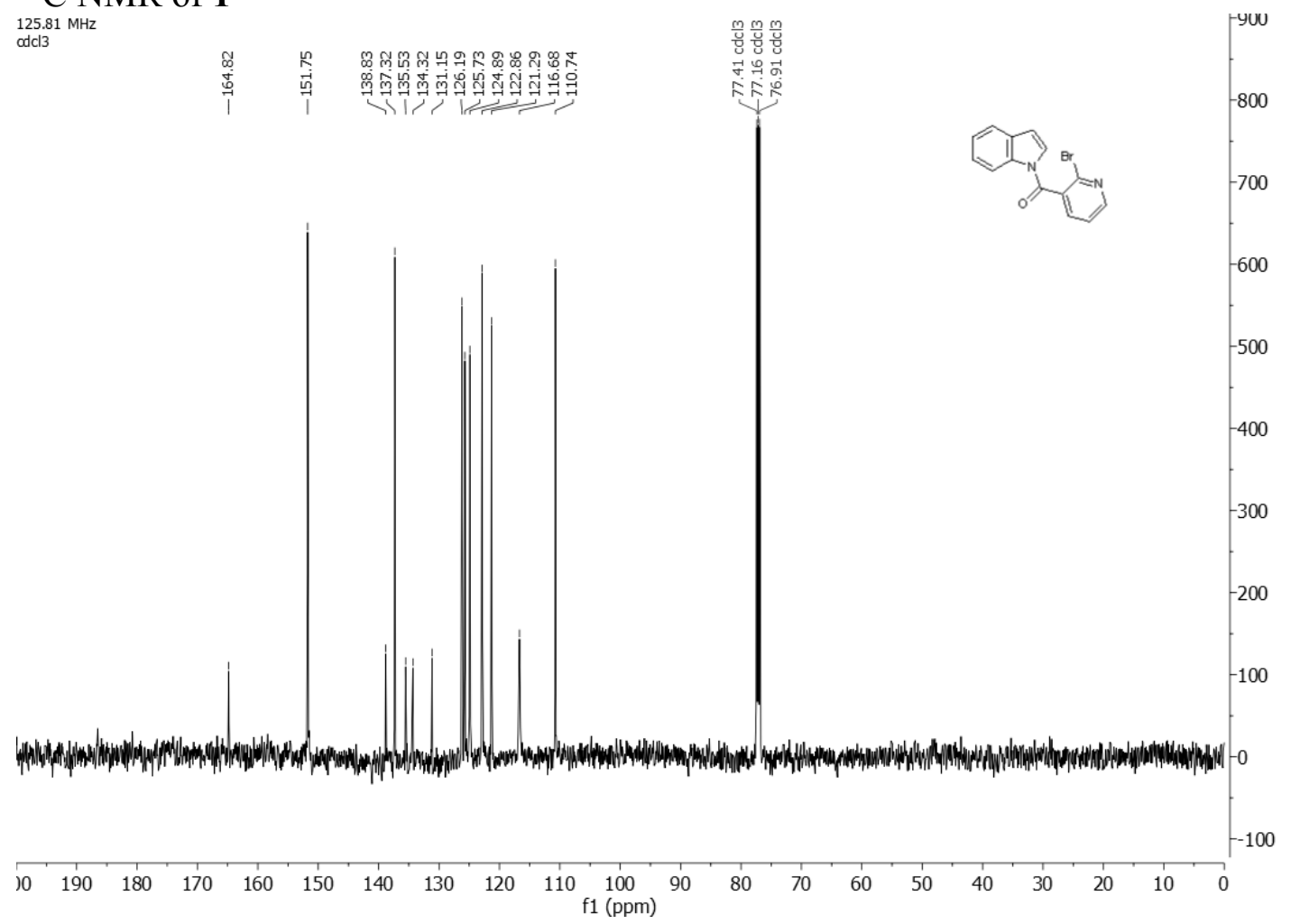




\section{${ }^{1} \mathrm{H}$ NMR of $\mathbf{S 2}$}

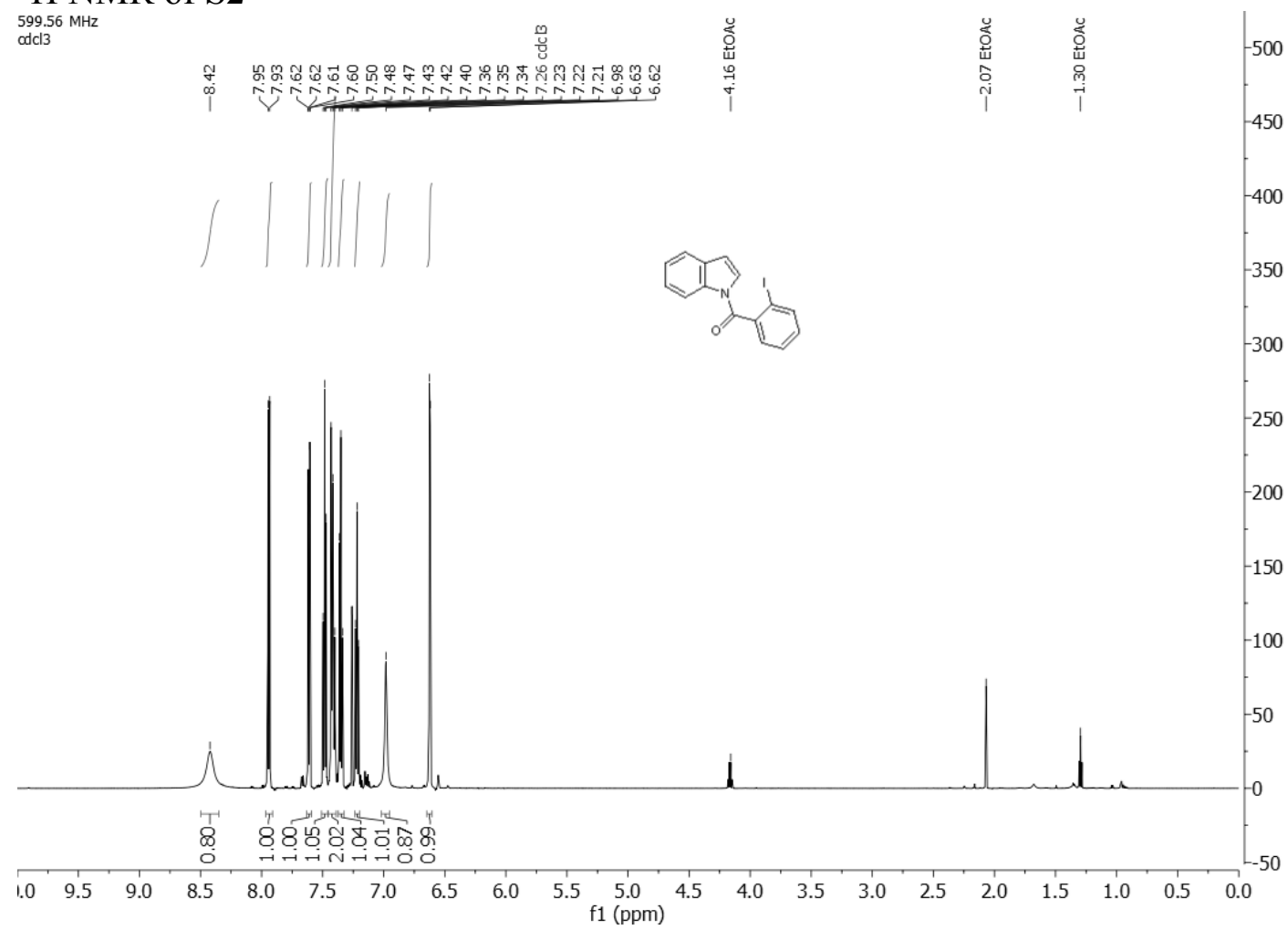

${ }^{13} \mathrm{C}$ NMR of $\mathbf{S 2}$

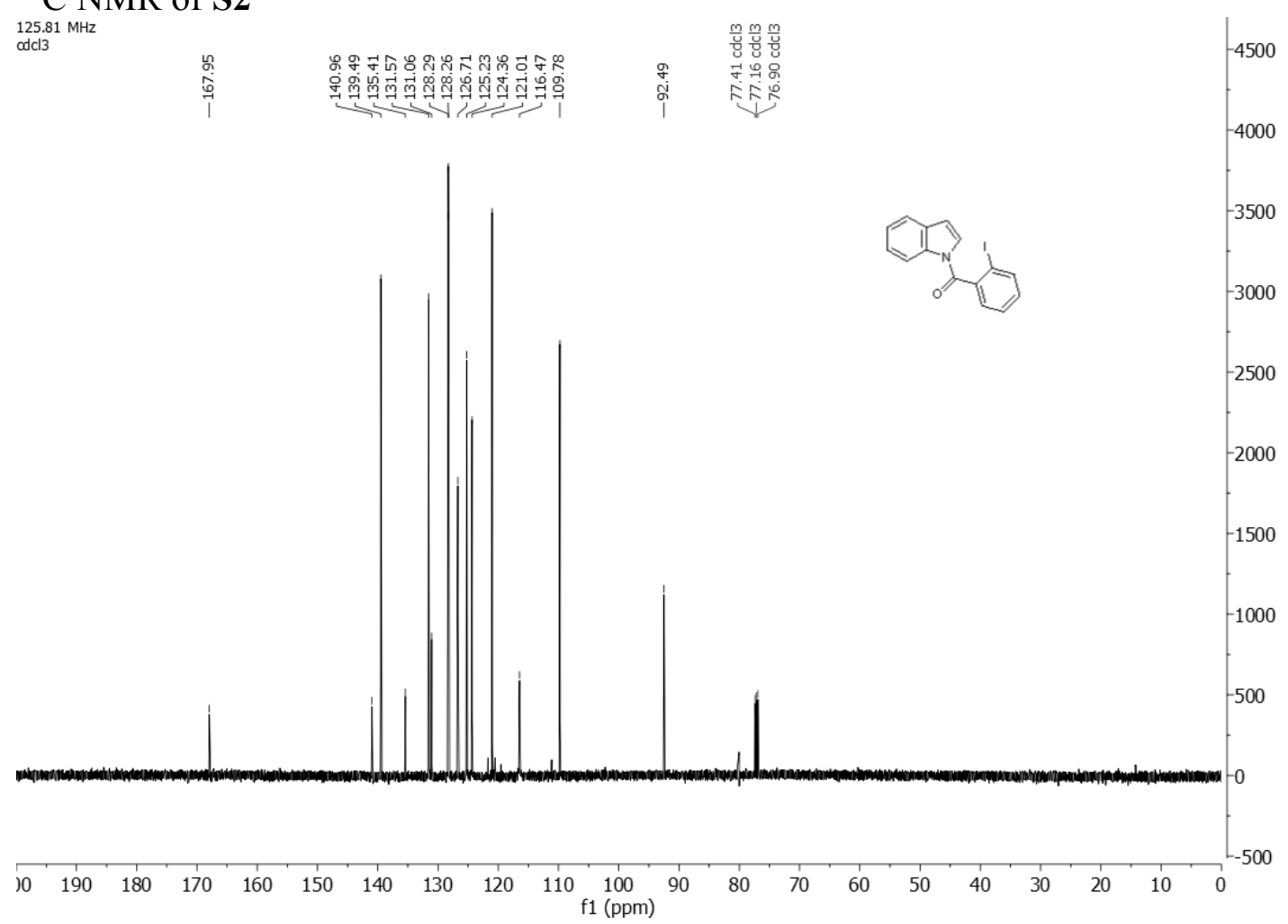




\section{${ }^{1} \mathrm{H}$ NMR of $\mathbf{S 3}$}

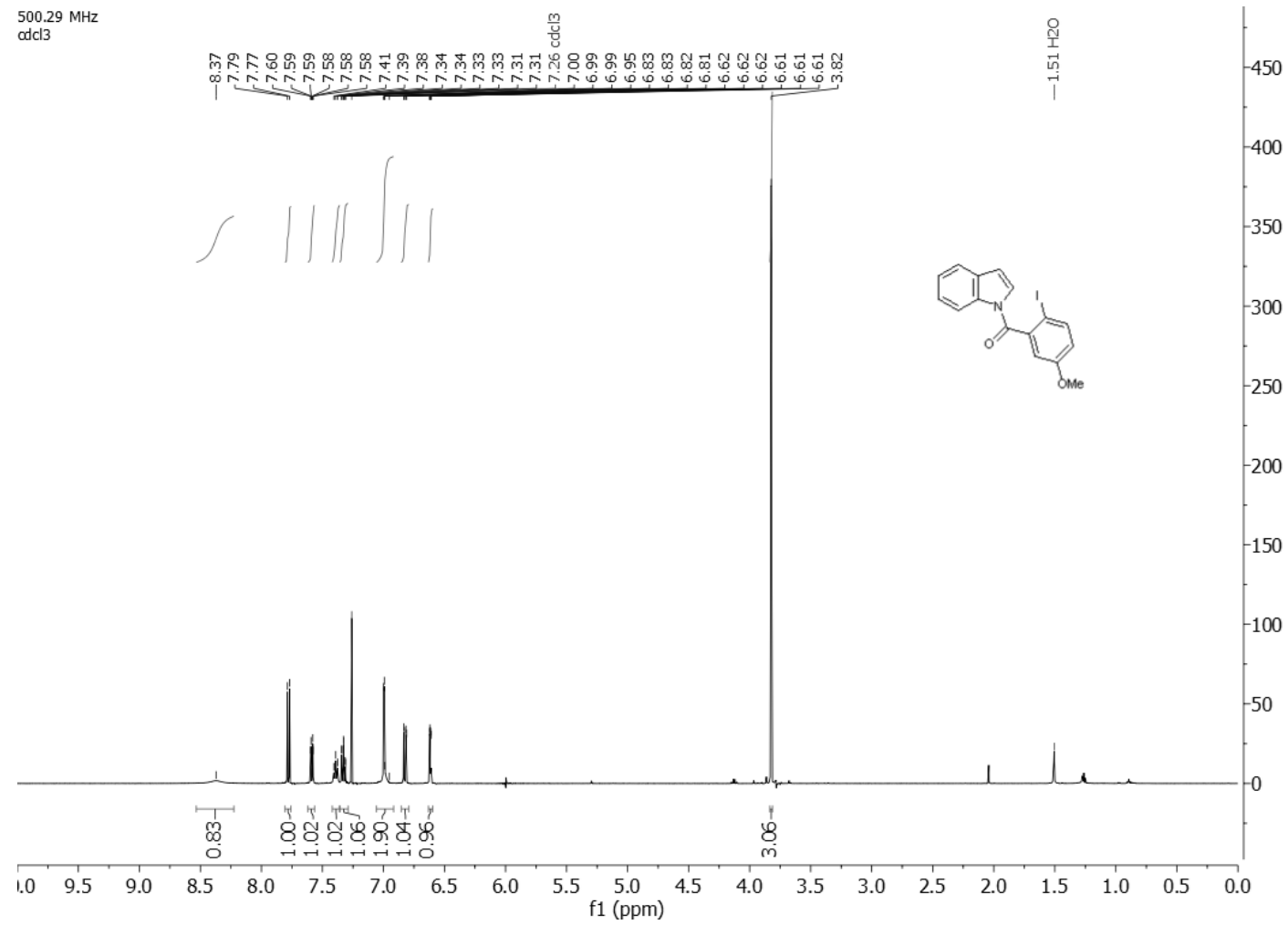

${ }^{13} \mathrm{C}$ NMR of $\mathbf{S 3}$

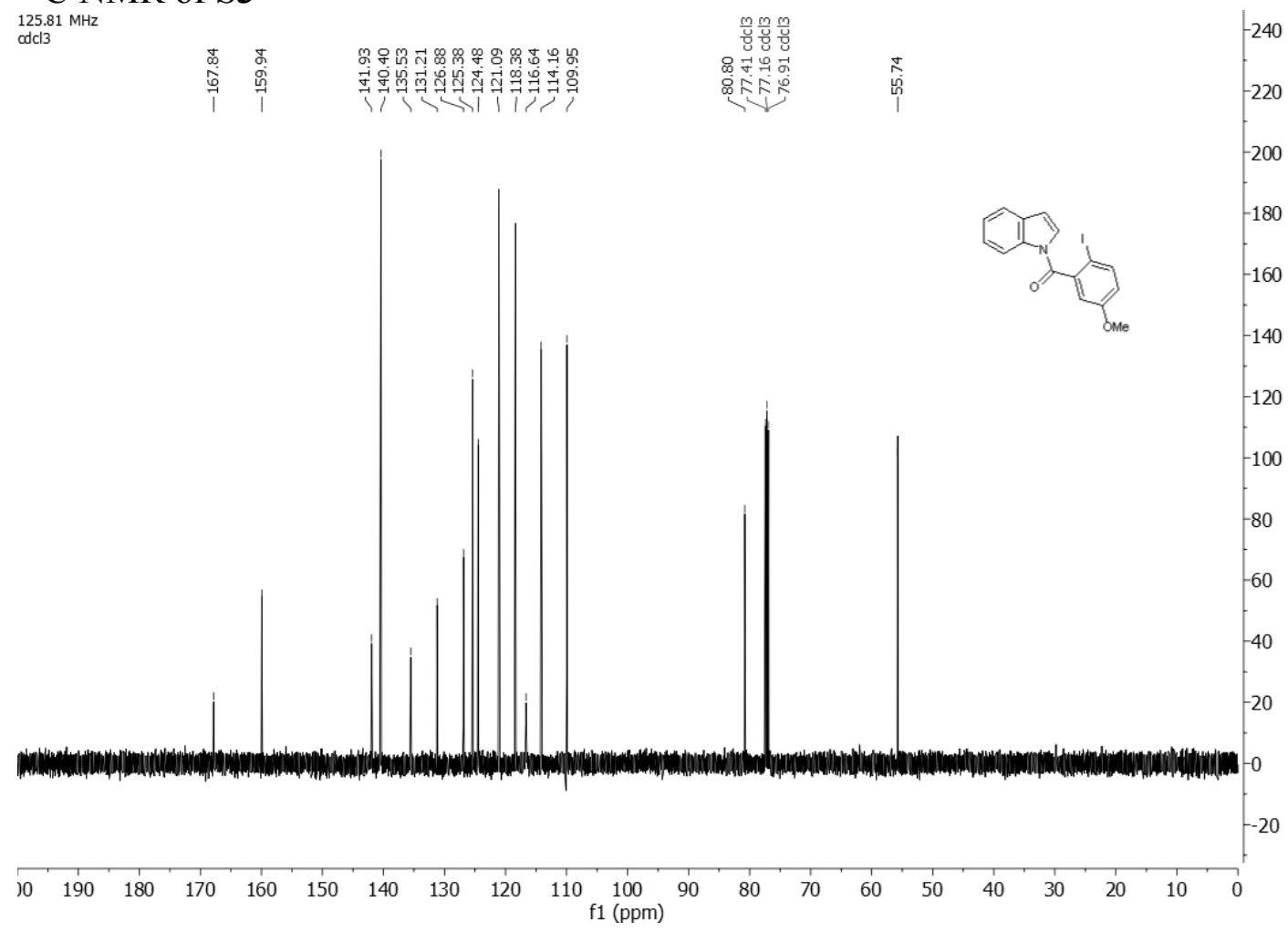




\section{${ }^{1} \mathrm{H}$ NMR of $\mathbf{S 4}$}

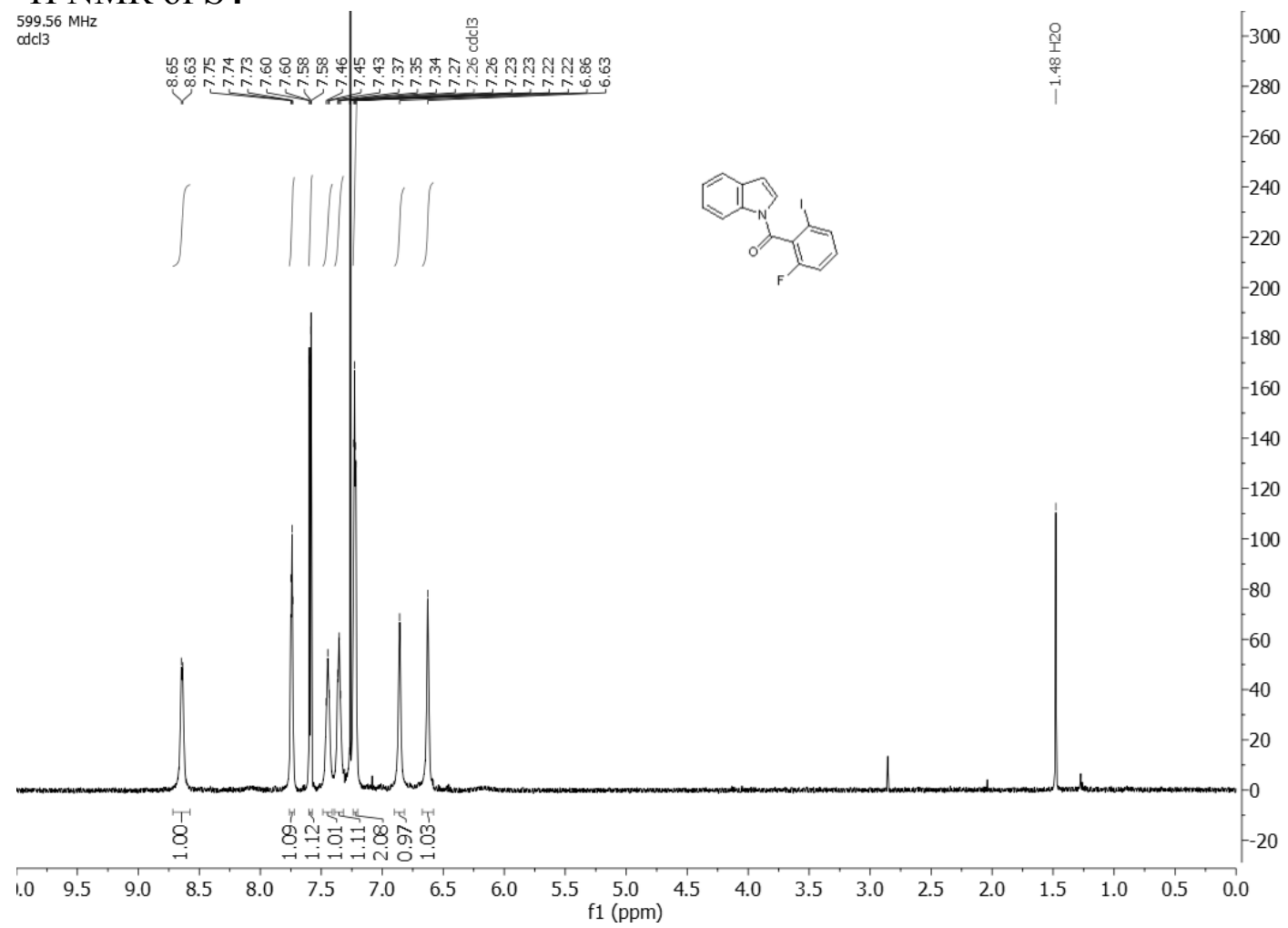

${ }^{13} \mathrm{C}$ NMR of S4

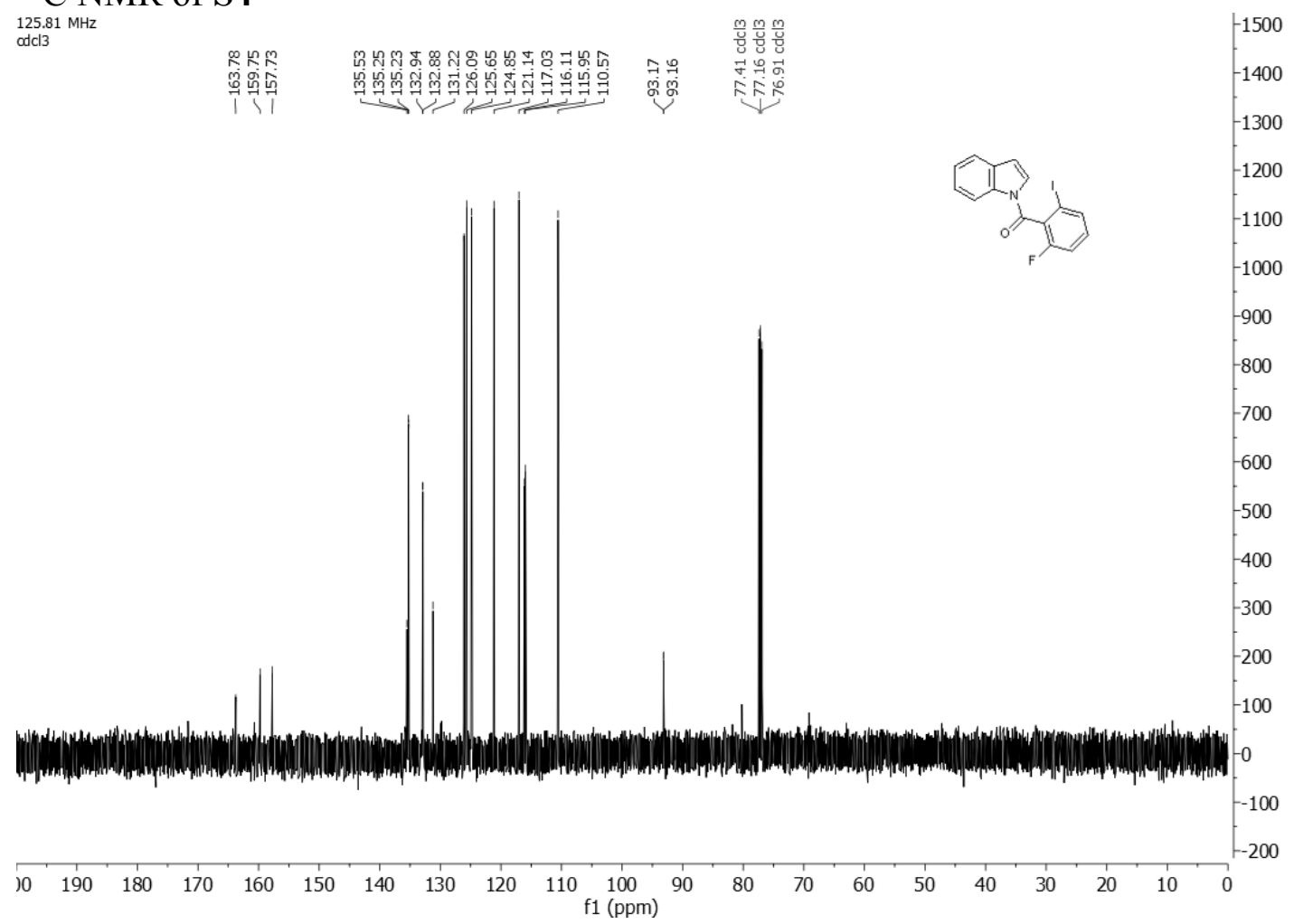




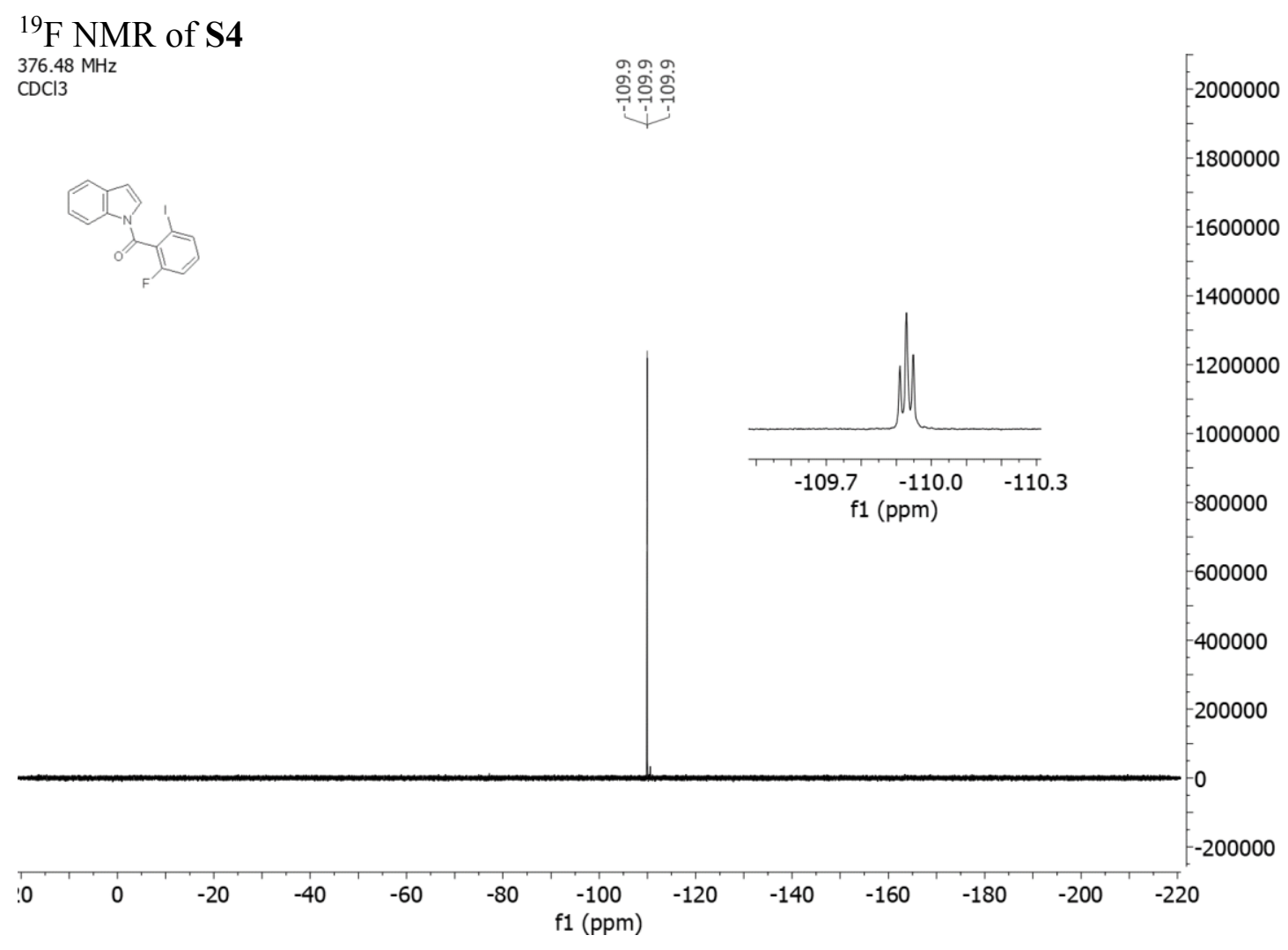




\section{${ }^{1} \mathrm{H}$ NMR of $\mathbf{S 5}$}

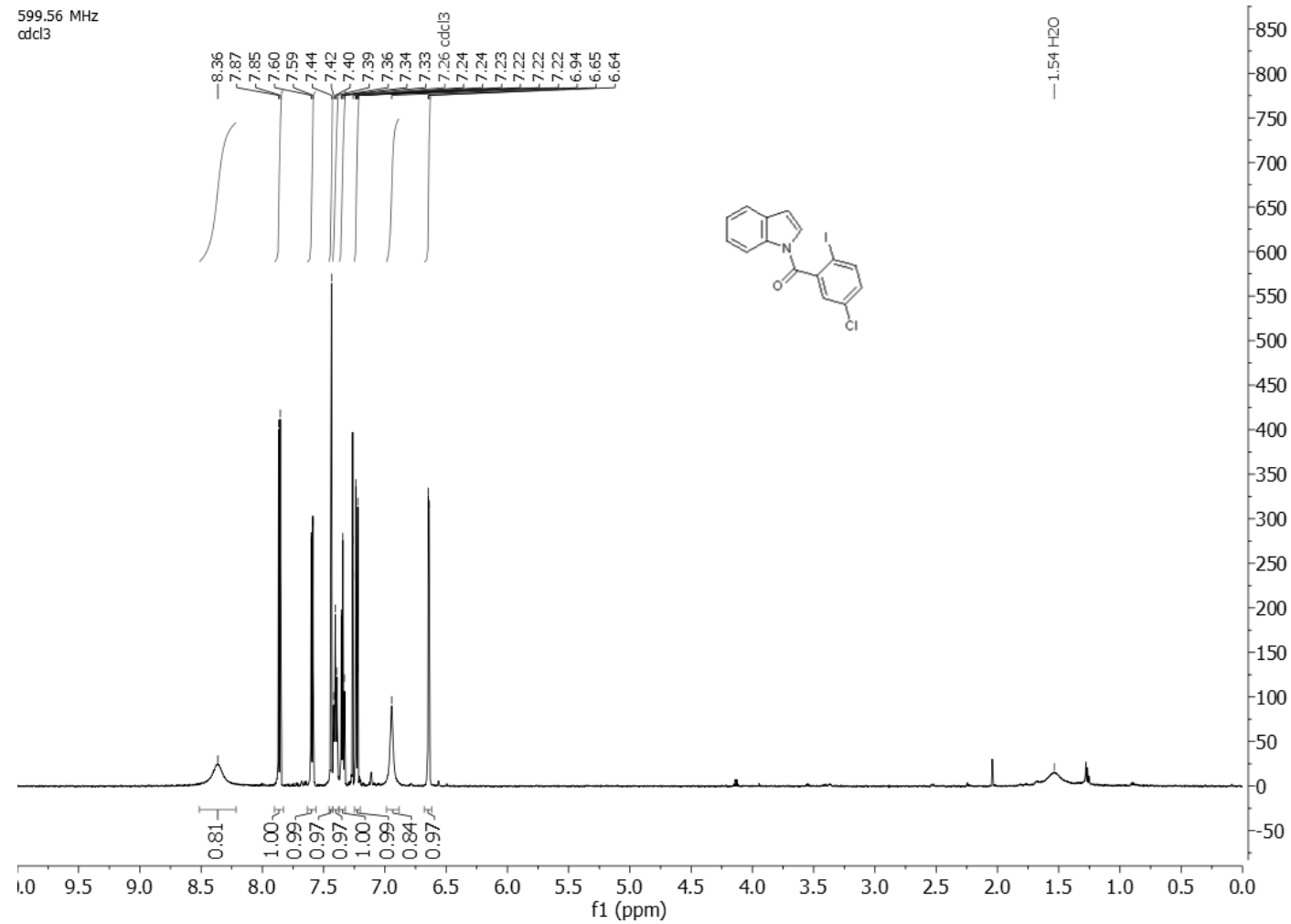

${ }^{13} \mathrm{C}$ NMR of $\mathbf{S 5}$

${ }_{0.1253}^{125.81} \mathrm{MHz}$

\begin{tabular}{|c|c|c|}
\hline 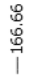 & 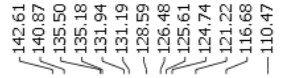 & 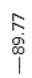 \\
\hline
\end{tabular}

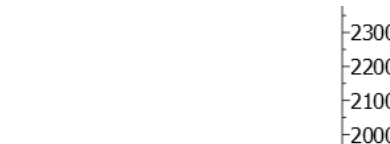

2000
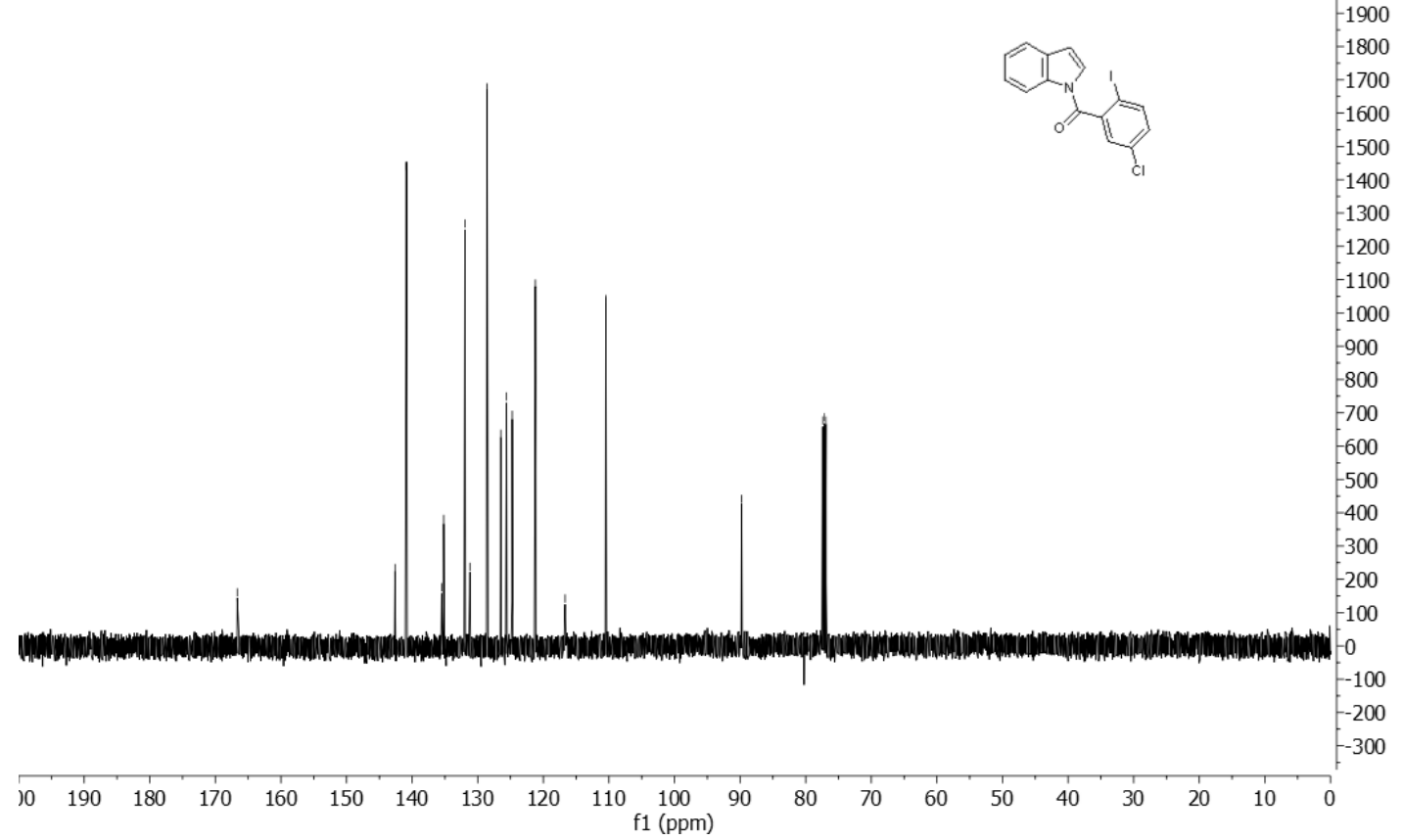


\section{${ }^{1} \mathrm{H}$ NMR of S6}

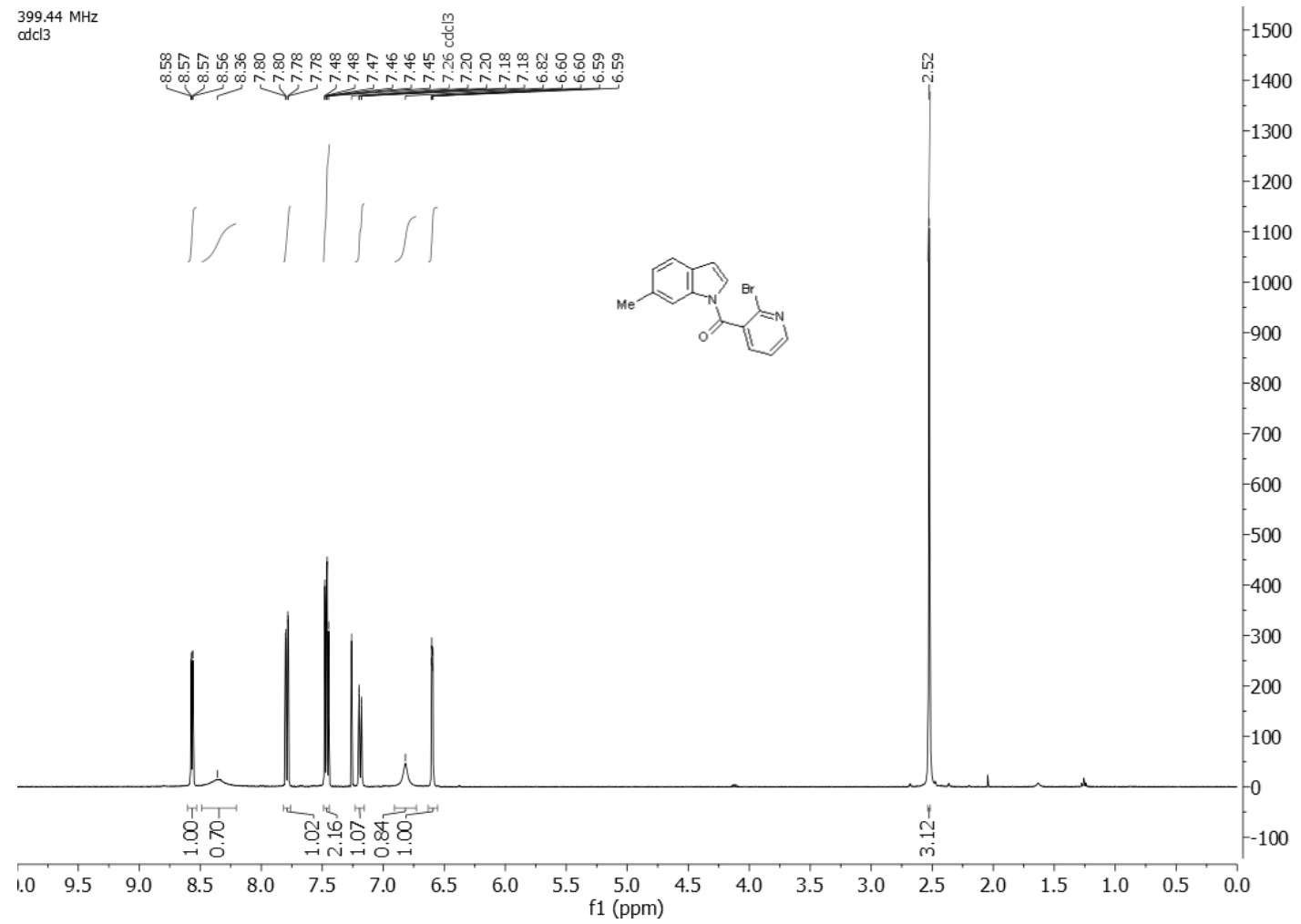

\section{${ }^{13} \mathrm{C}$ NMR of $\mathbf{S 6}$}

$125.81 \mathrm{MHz}$
oddl3

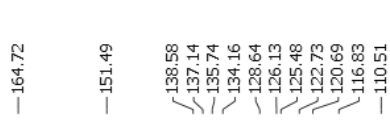

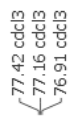

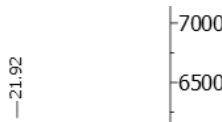

$-6000$

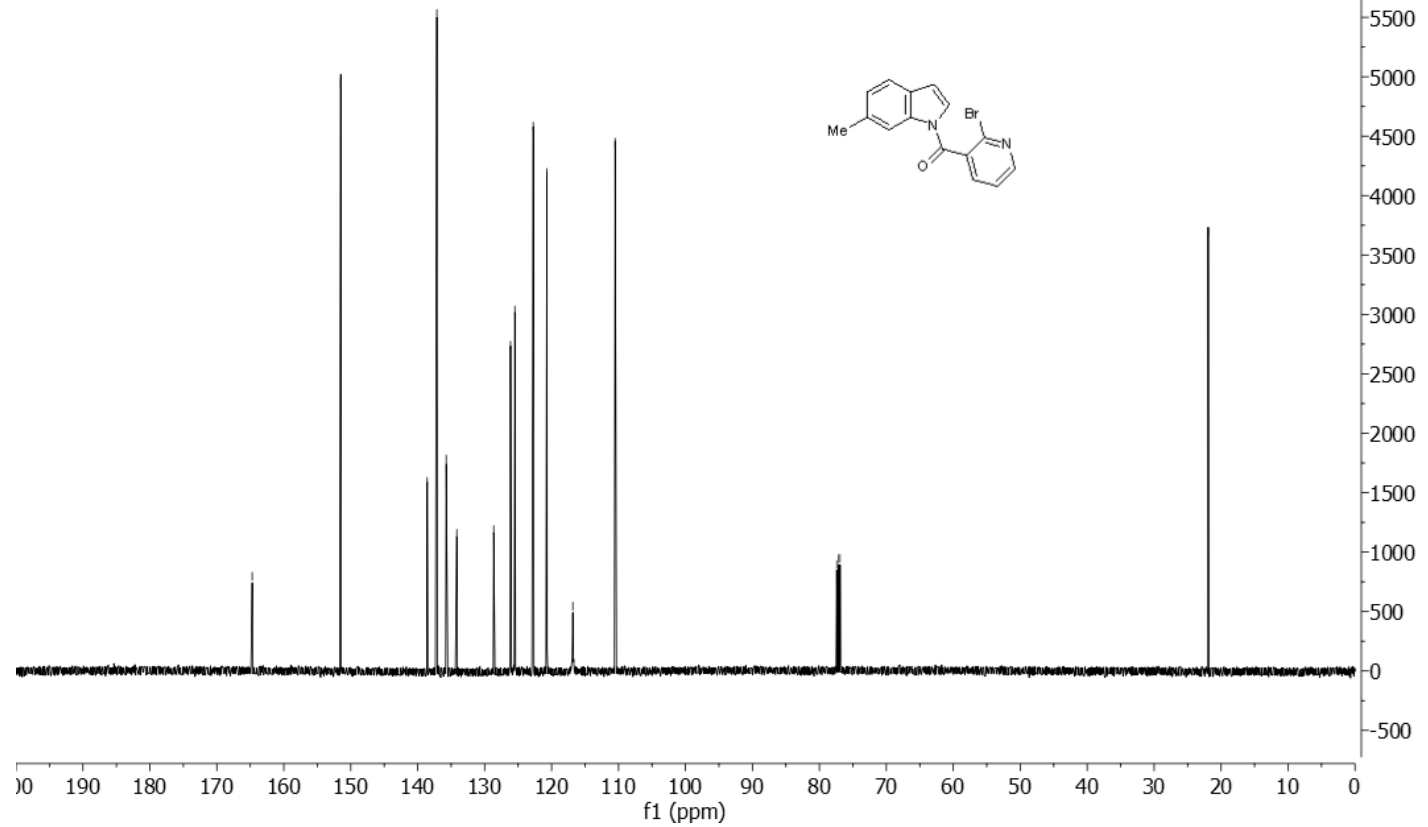




\section{${ }^{1} \mathrm{H}$ NMR of $\mathbf{S 7}$}

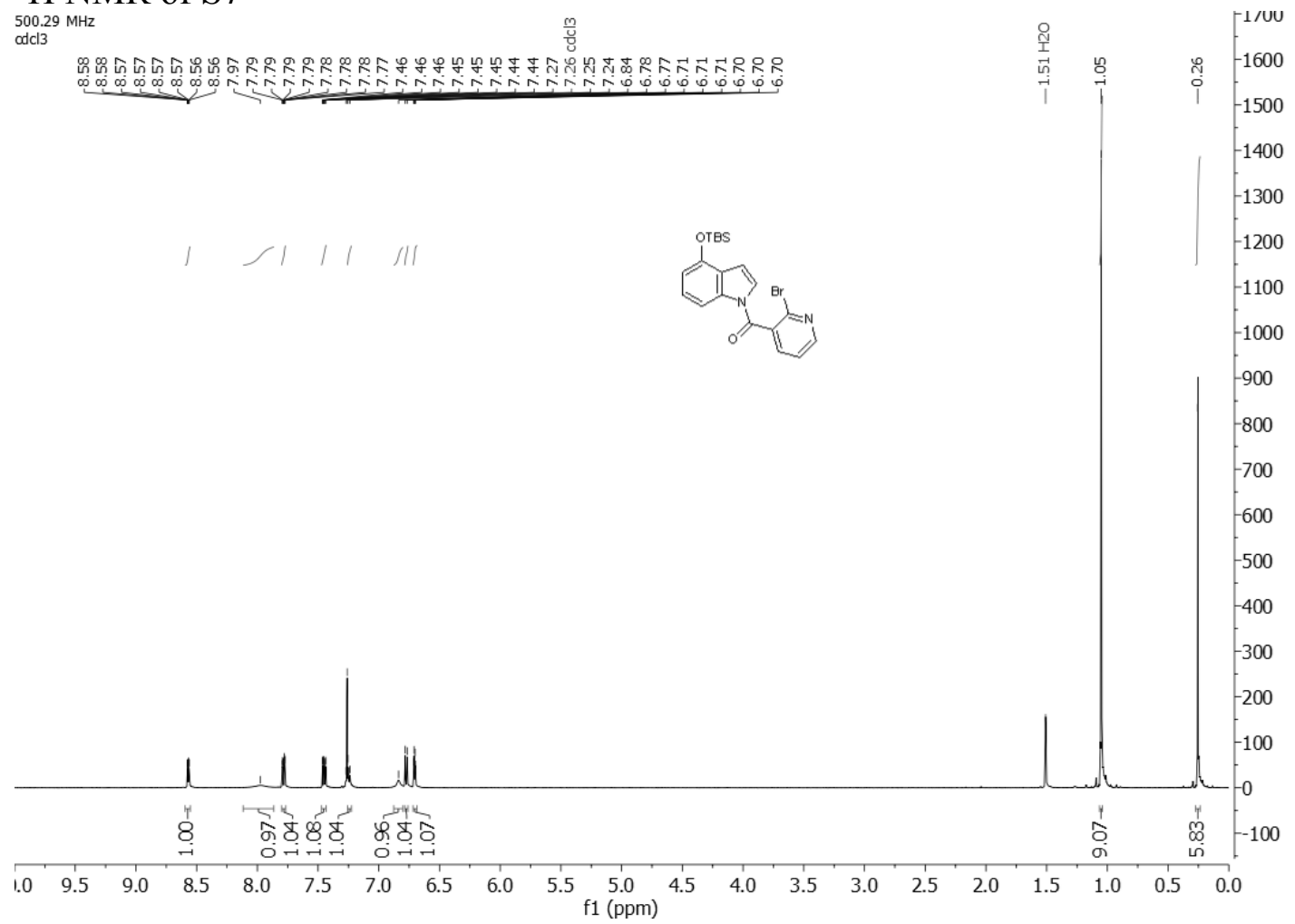

\section{${ }^{13} \mathrm{C}$ NMR of $\mathbf{S 7}$}

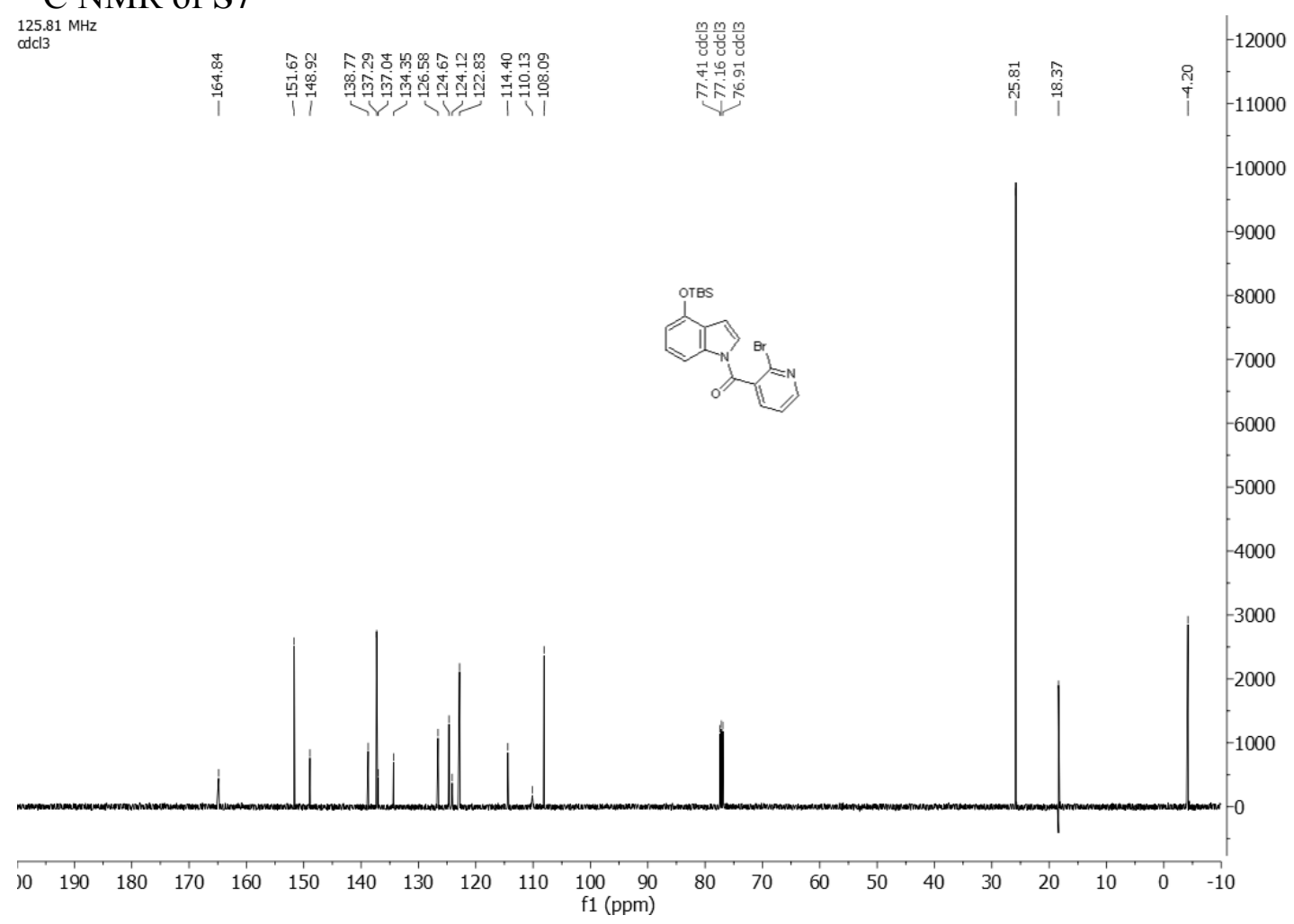


${ }^{1} \mathrm{H}$ NMR of $\mathbf{S 8}$

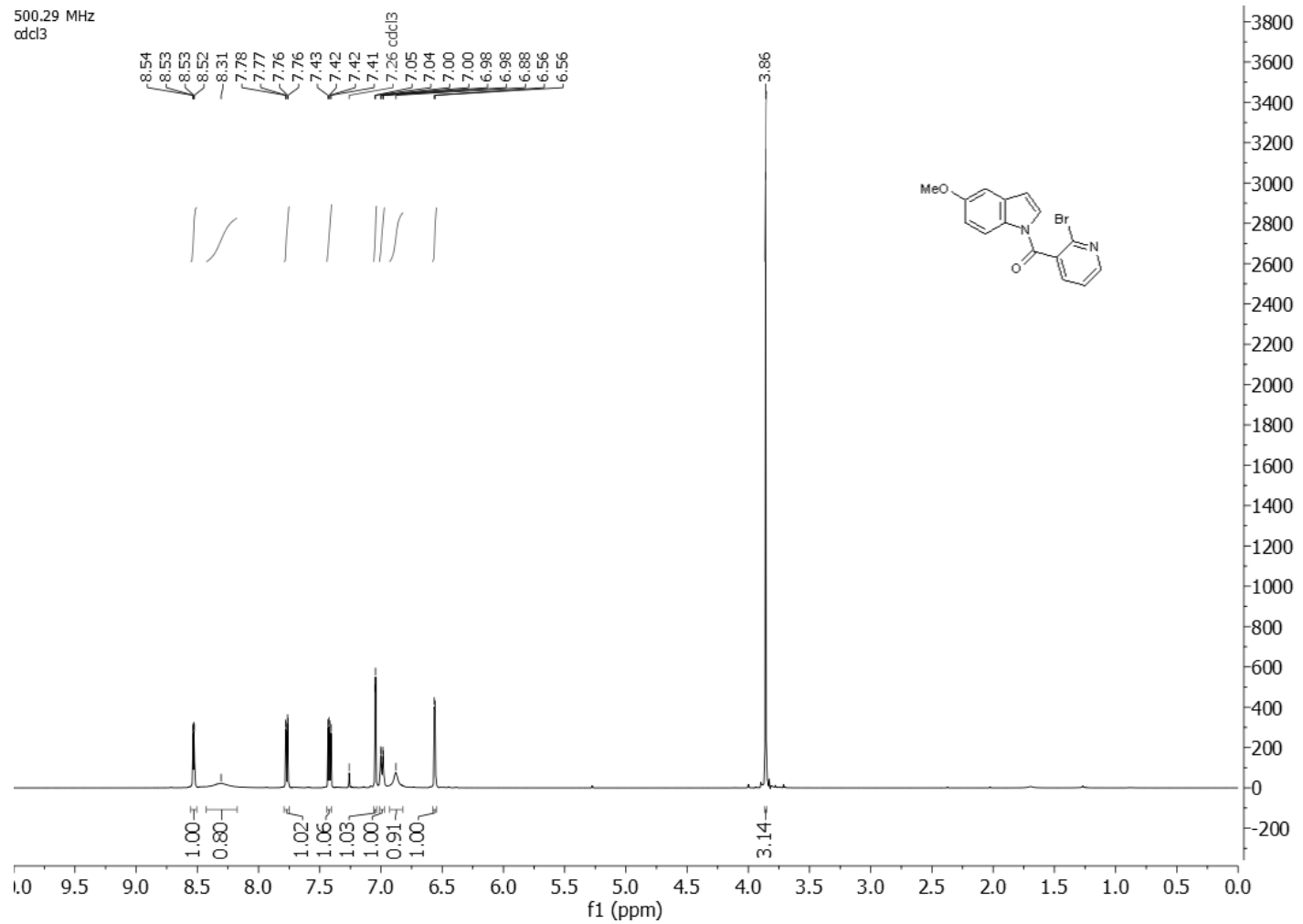

\section{${ }^{13} \mathrm{C}$ NMR of $\mathbf{S 8}$}

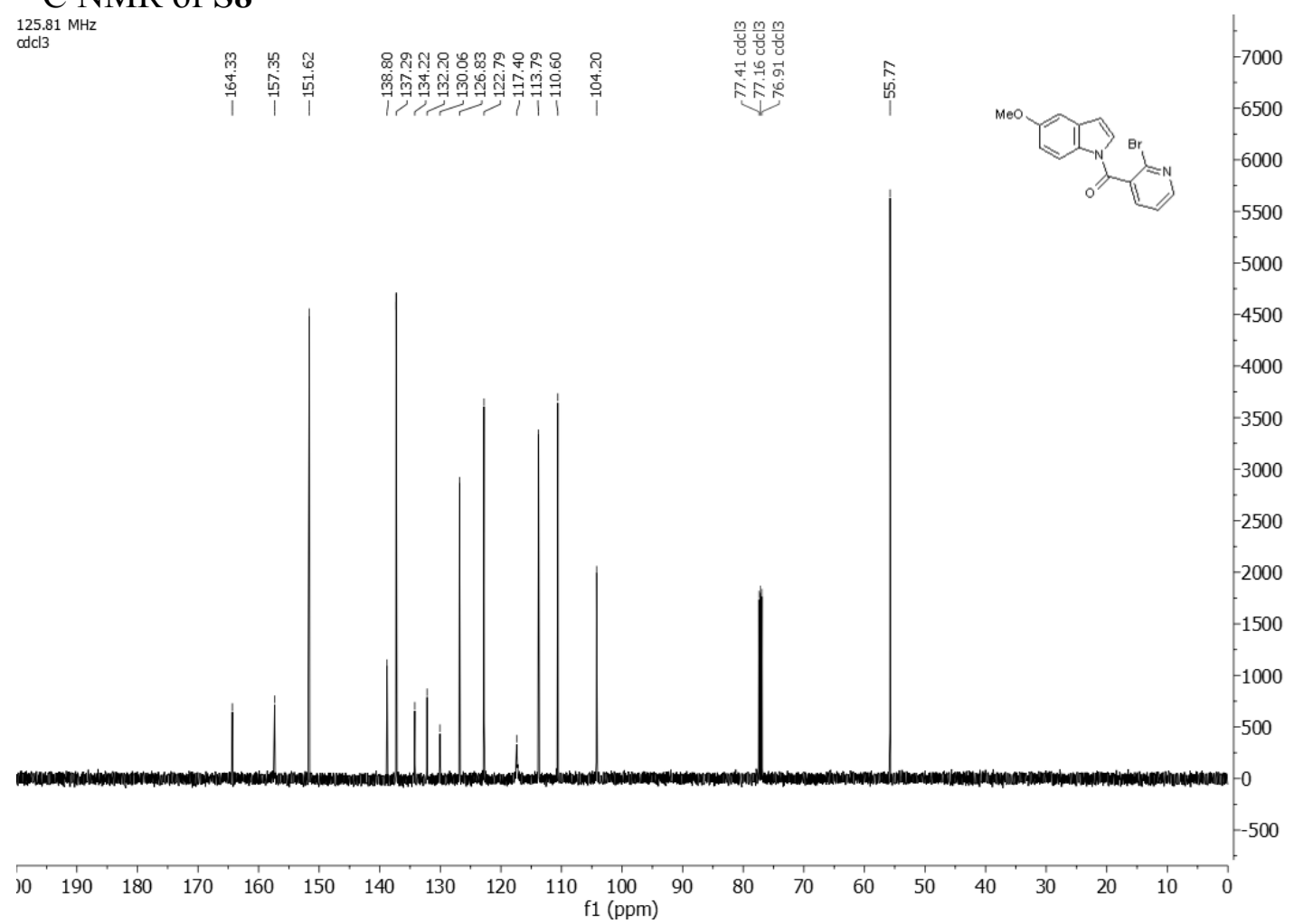

S-33 


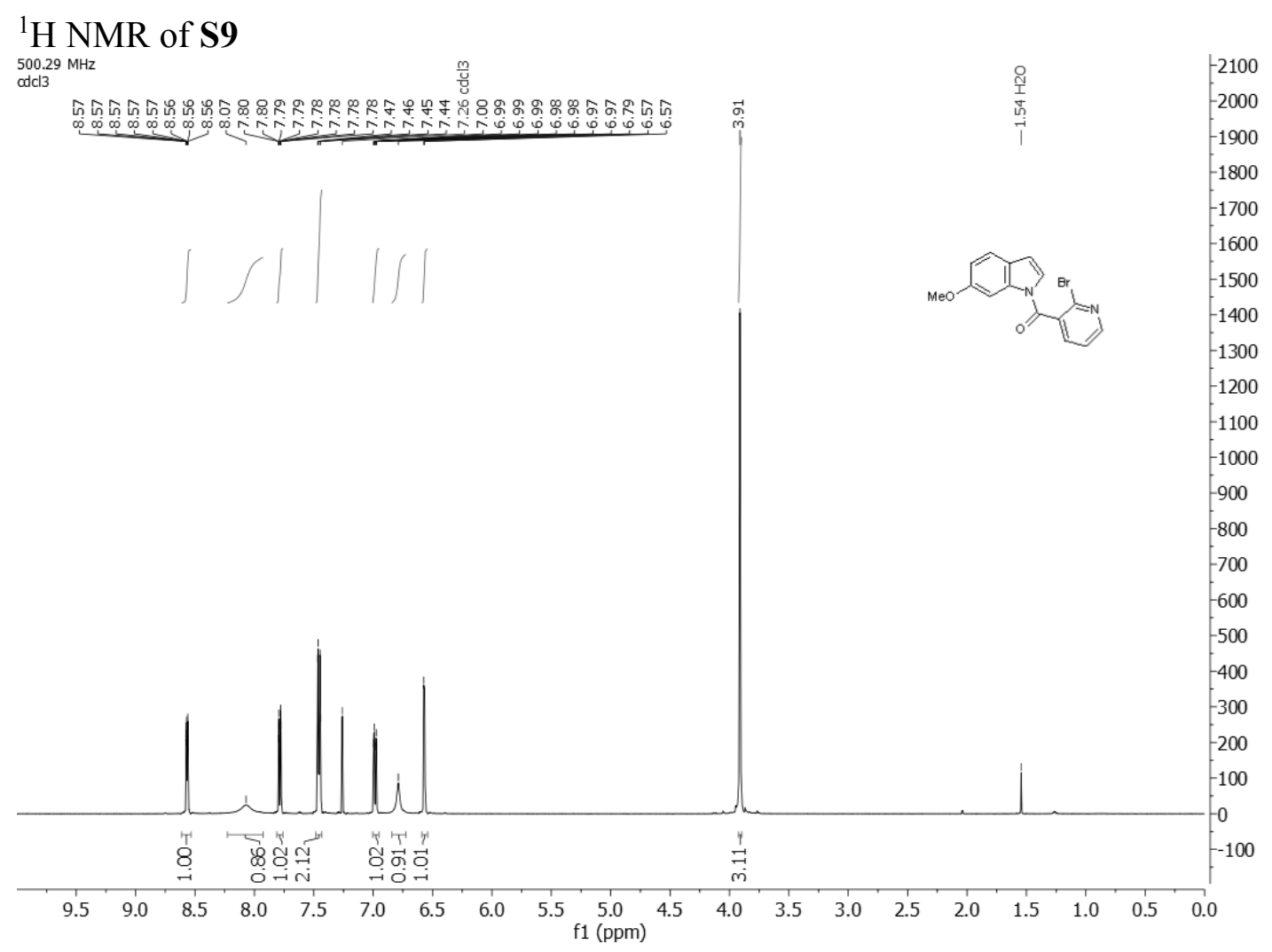

${ }^{13} \mathrm{C}$ NMR of $\mathbf{S 9}$

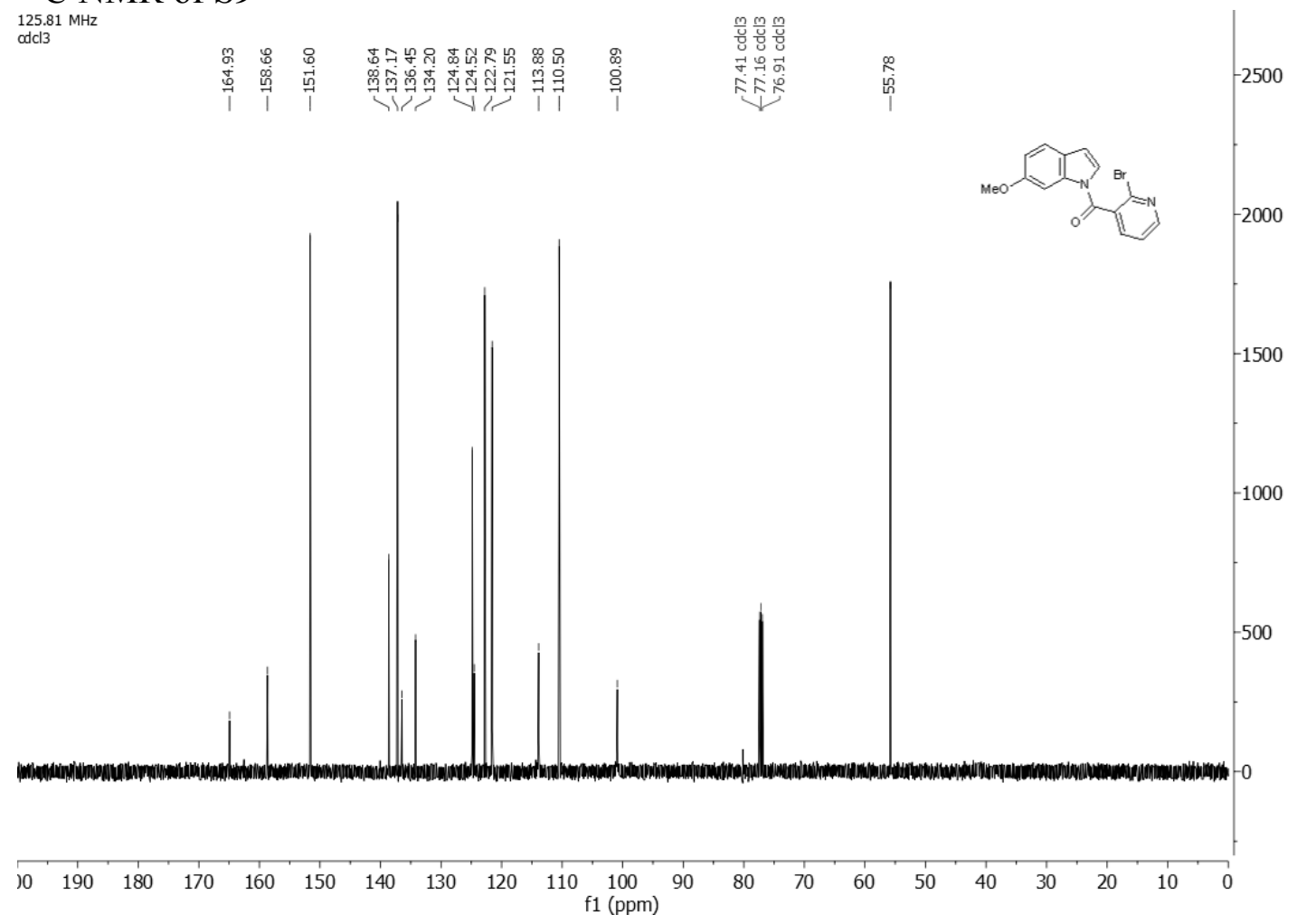




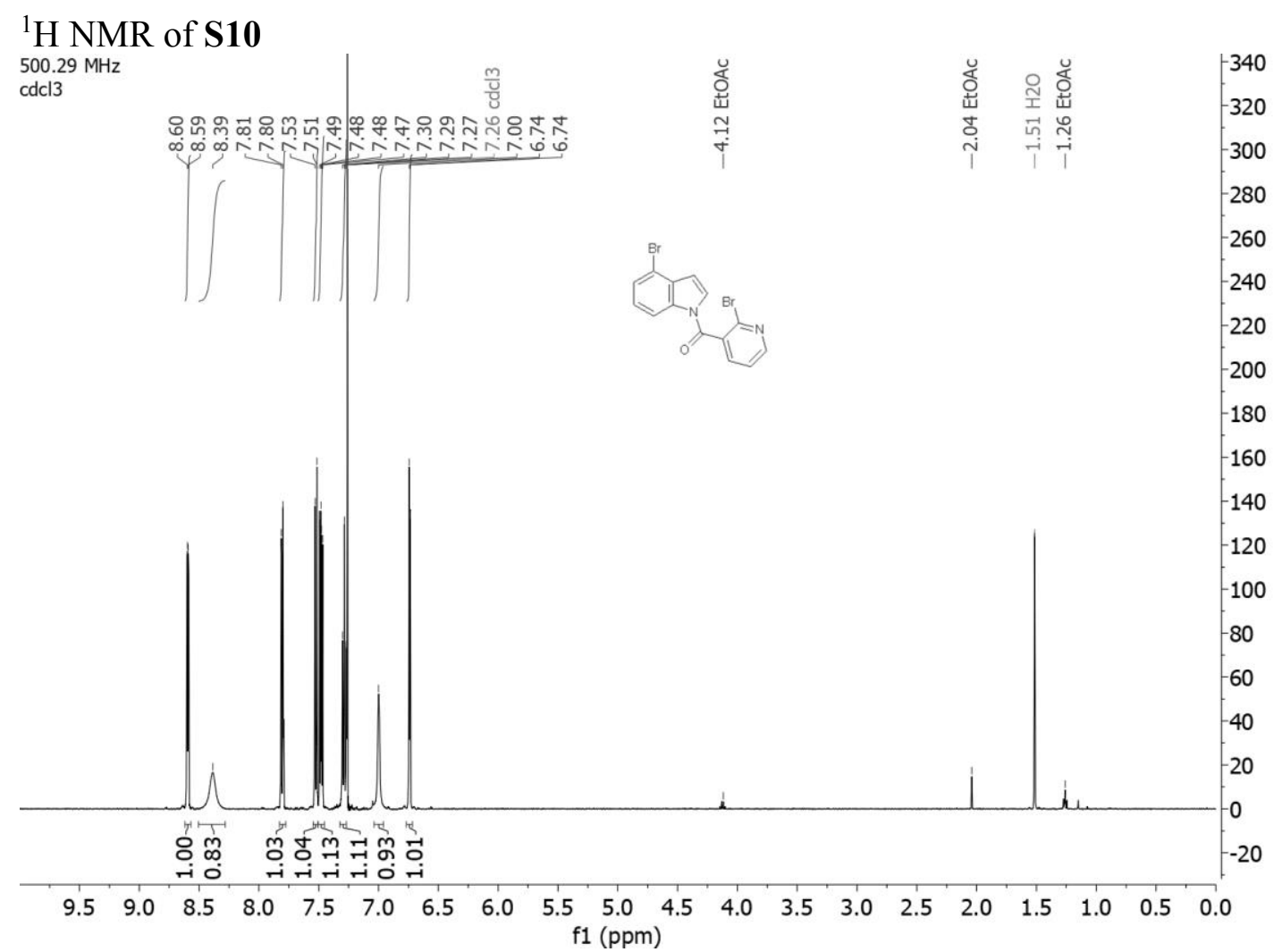

${ }^{13} \mathrm{C}$ NMR of $\mathbf{S 1 0}$

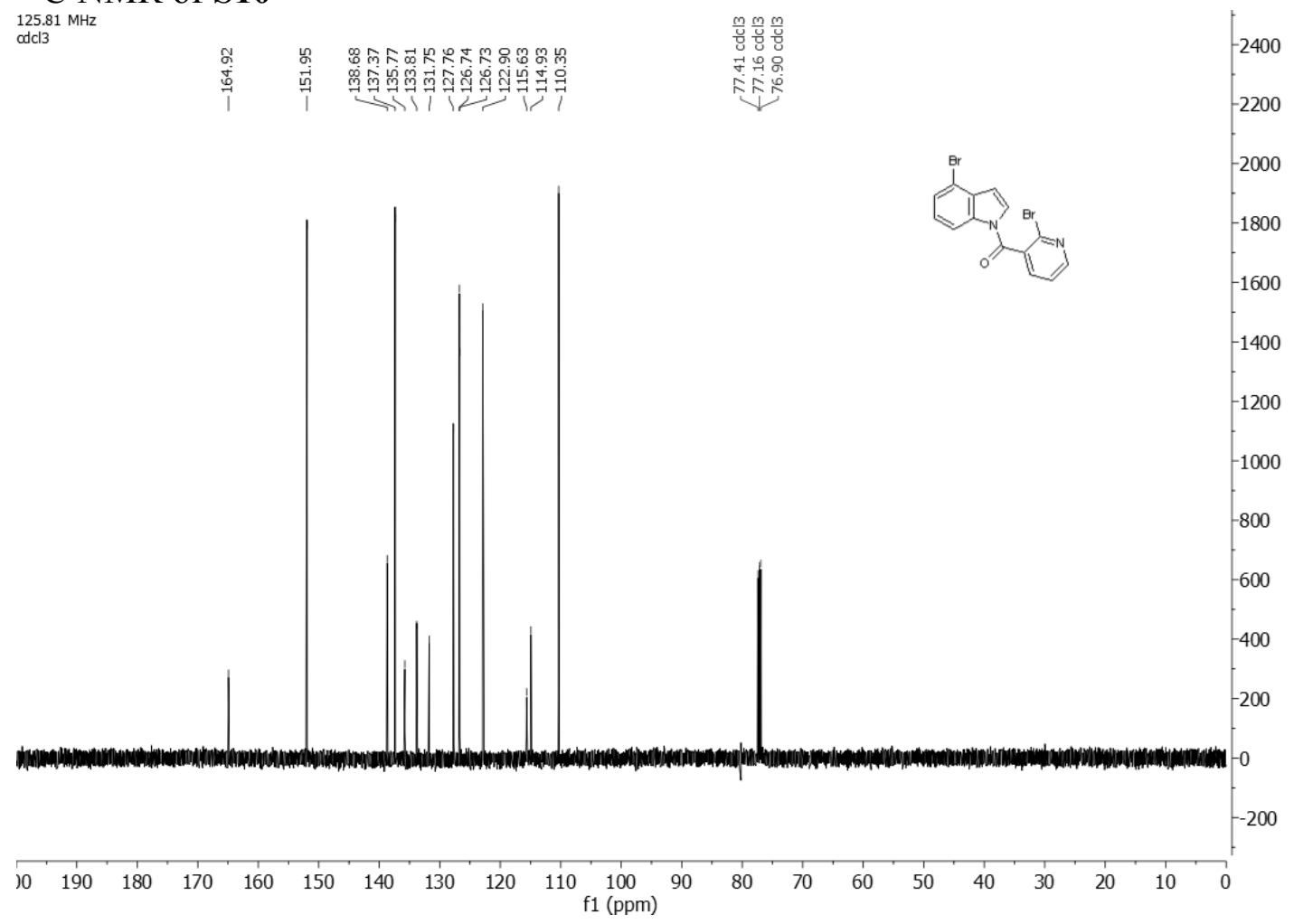




\section{${ }^{1} \mathrm{H}$ NMR of $\mathbf{S 1 1}$}

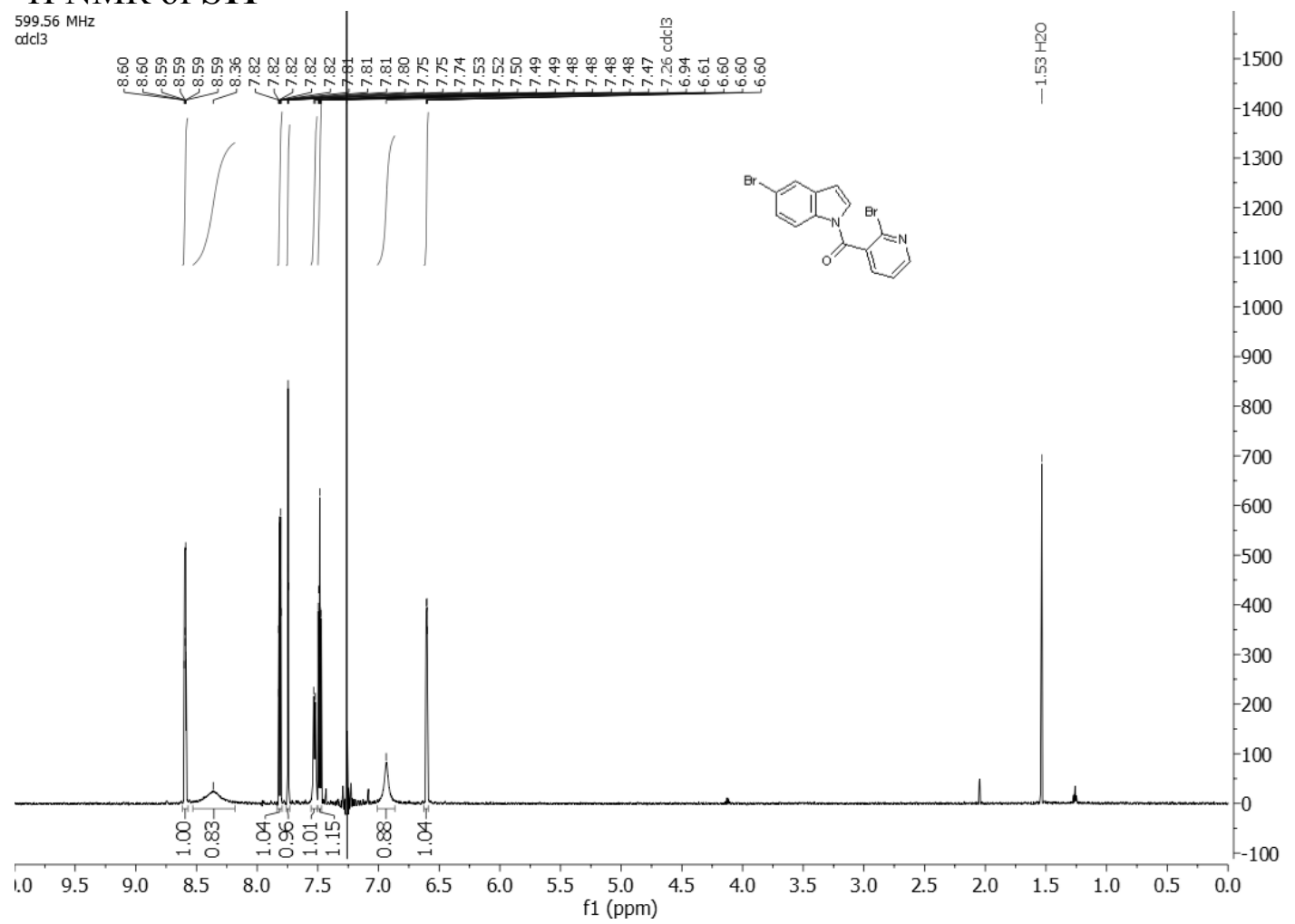

${ }^{13} \mathrm{C}$ NMR of $\mathbf{S 1 1}$

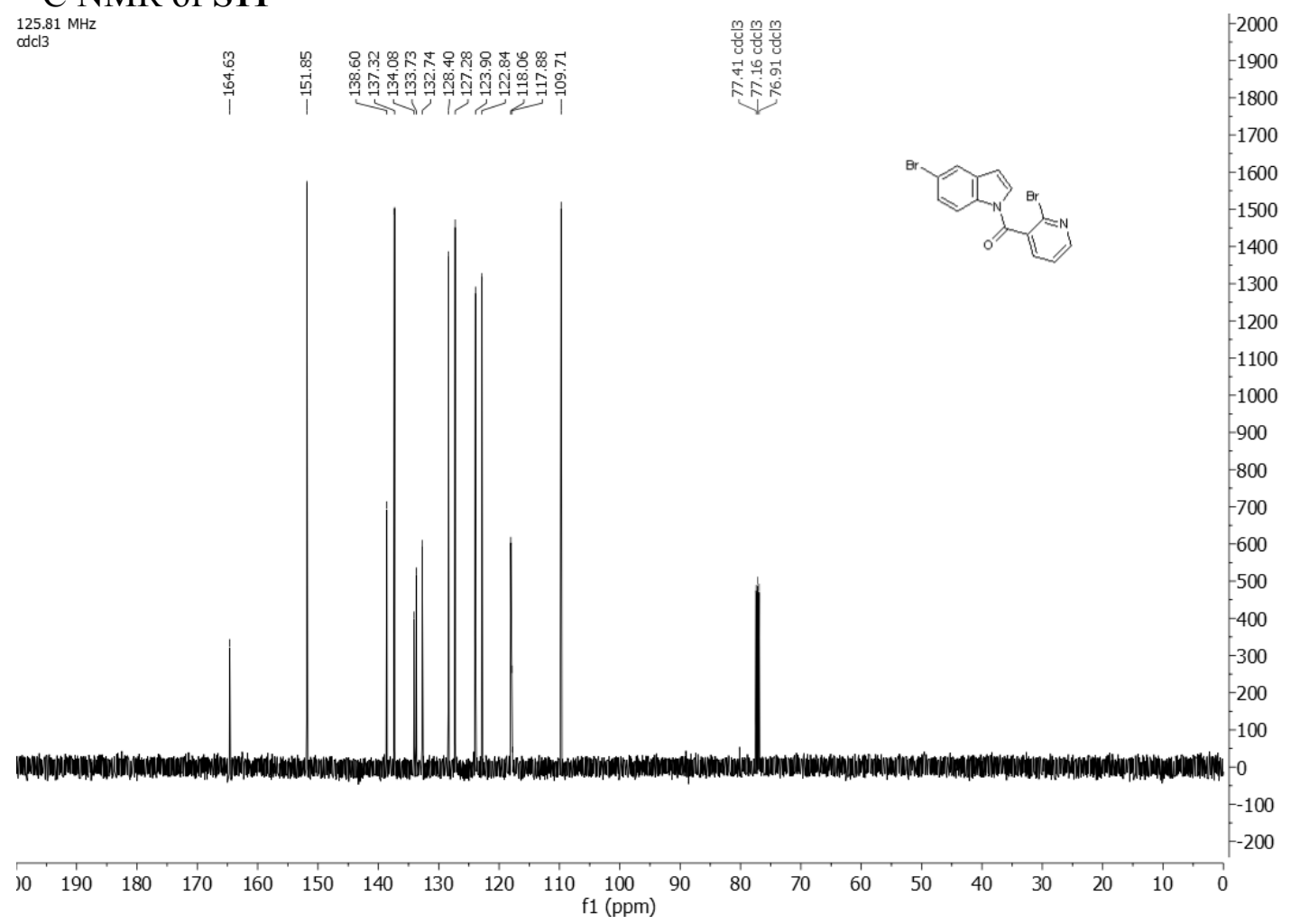


${ }^{1} \mathrm{H}$ NMR of $\mathbf{S 1 2}$

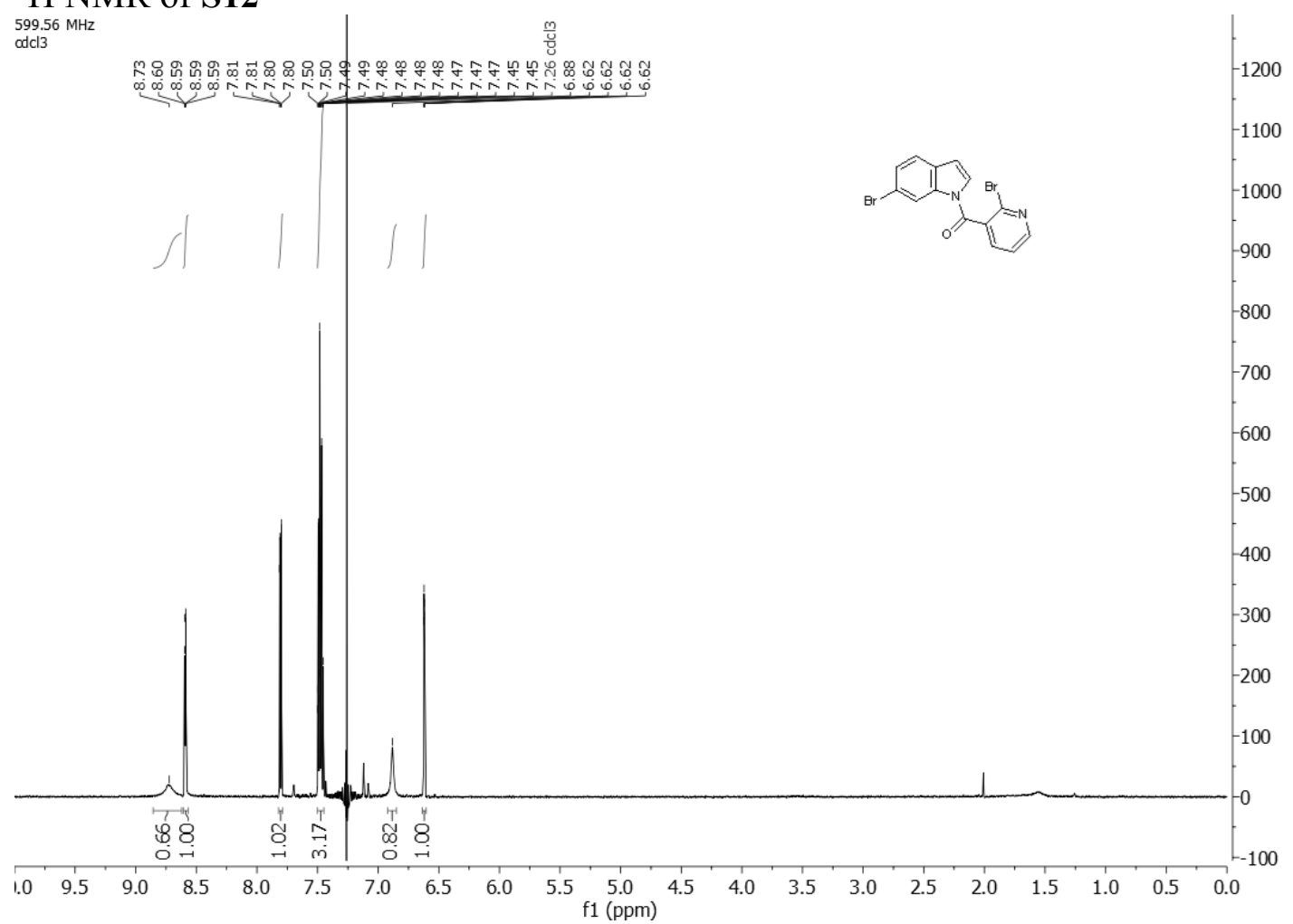

${ }^{13} \mathrm{C}$ NMR of $\mathbf{S 1 2}$

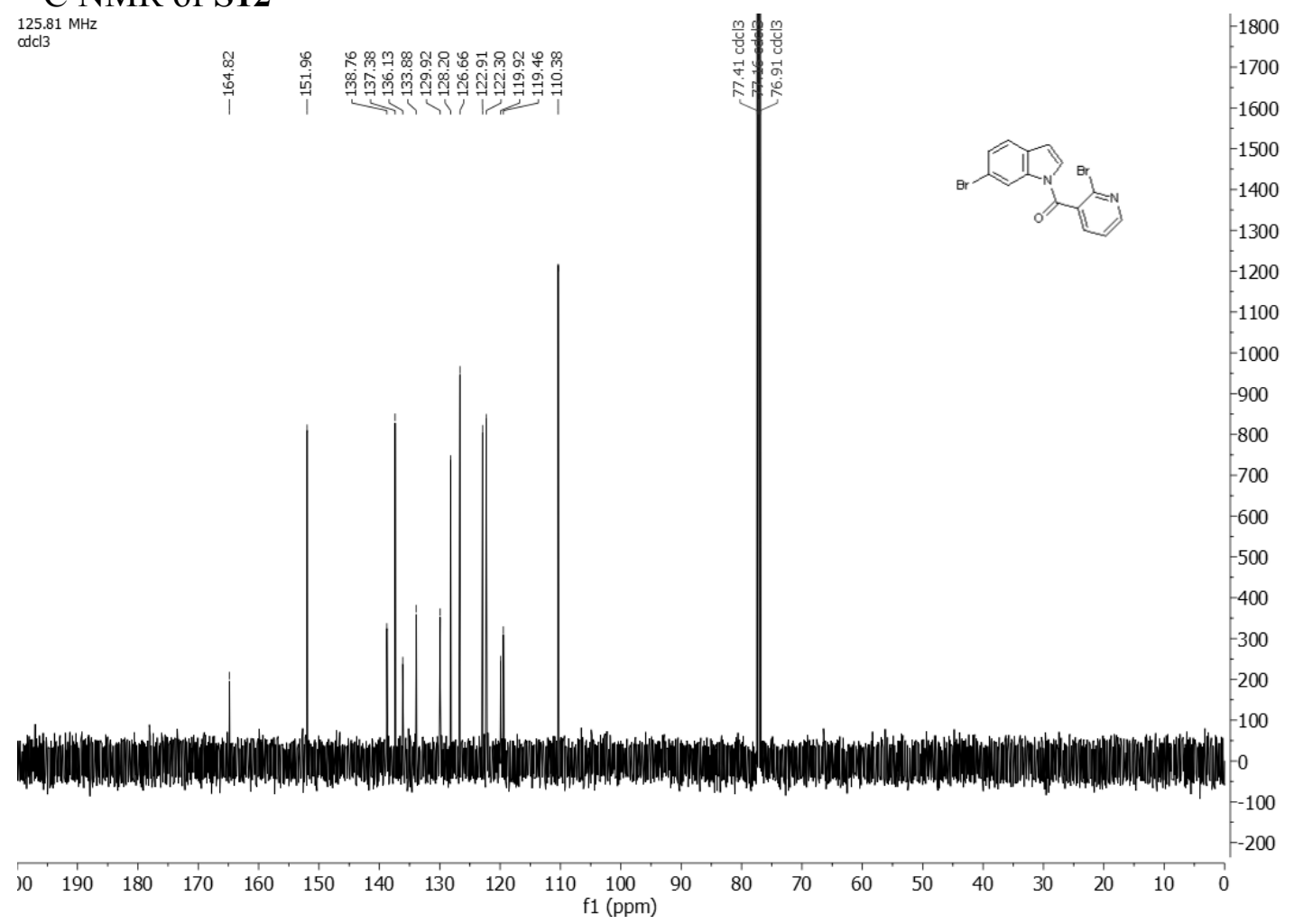




\section{${ }^{1} \mathrm{H}$ NMR of 2}

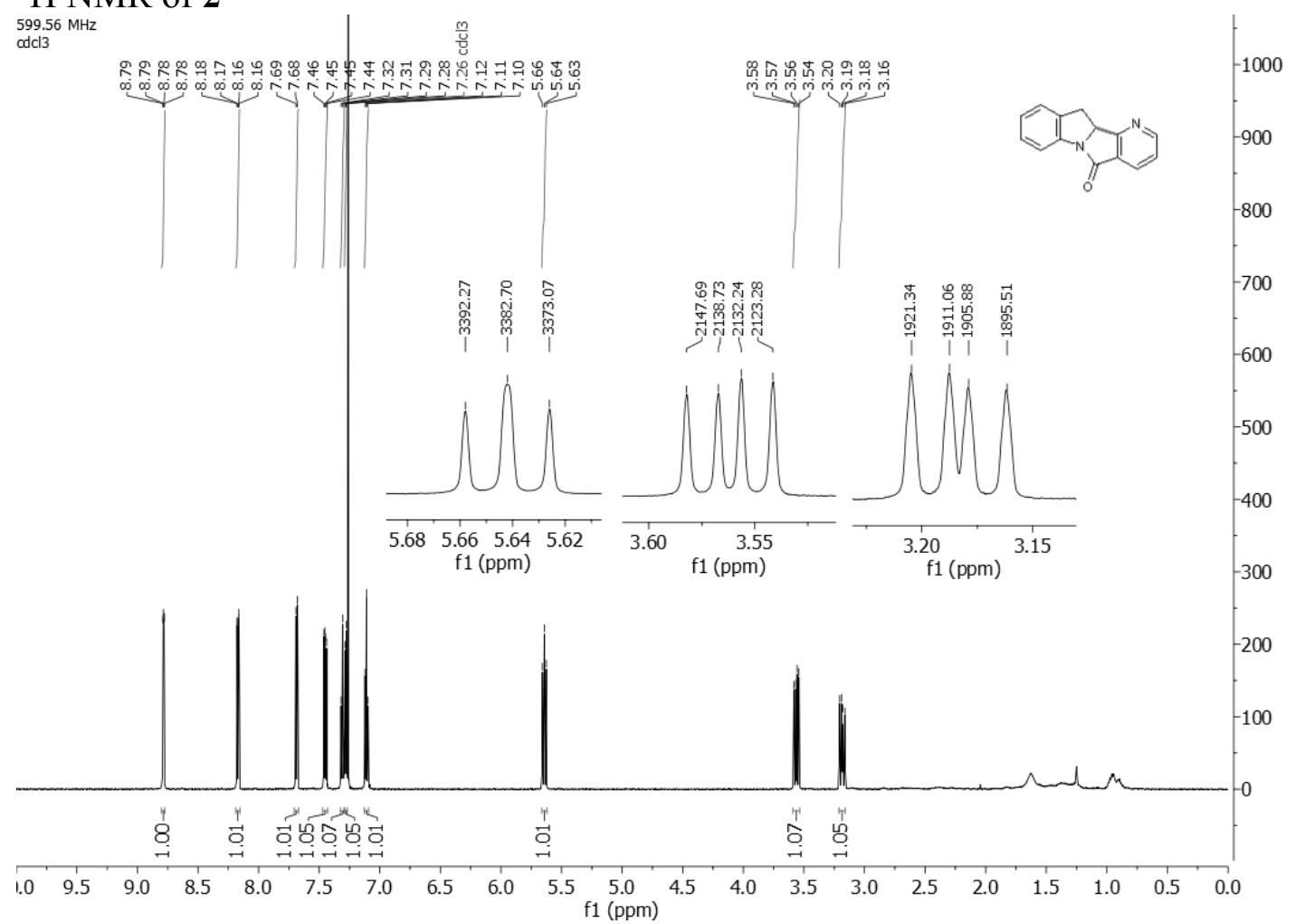

\section{${ }^{13} \mathrm{C}$ NMR of 2}

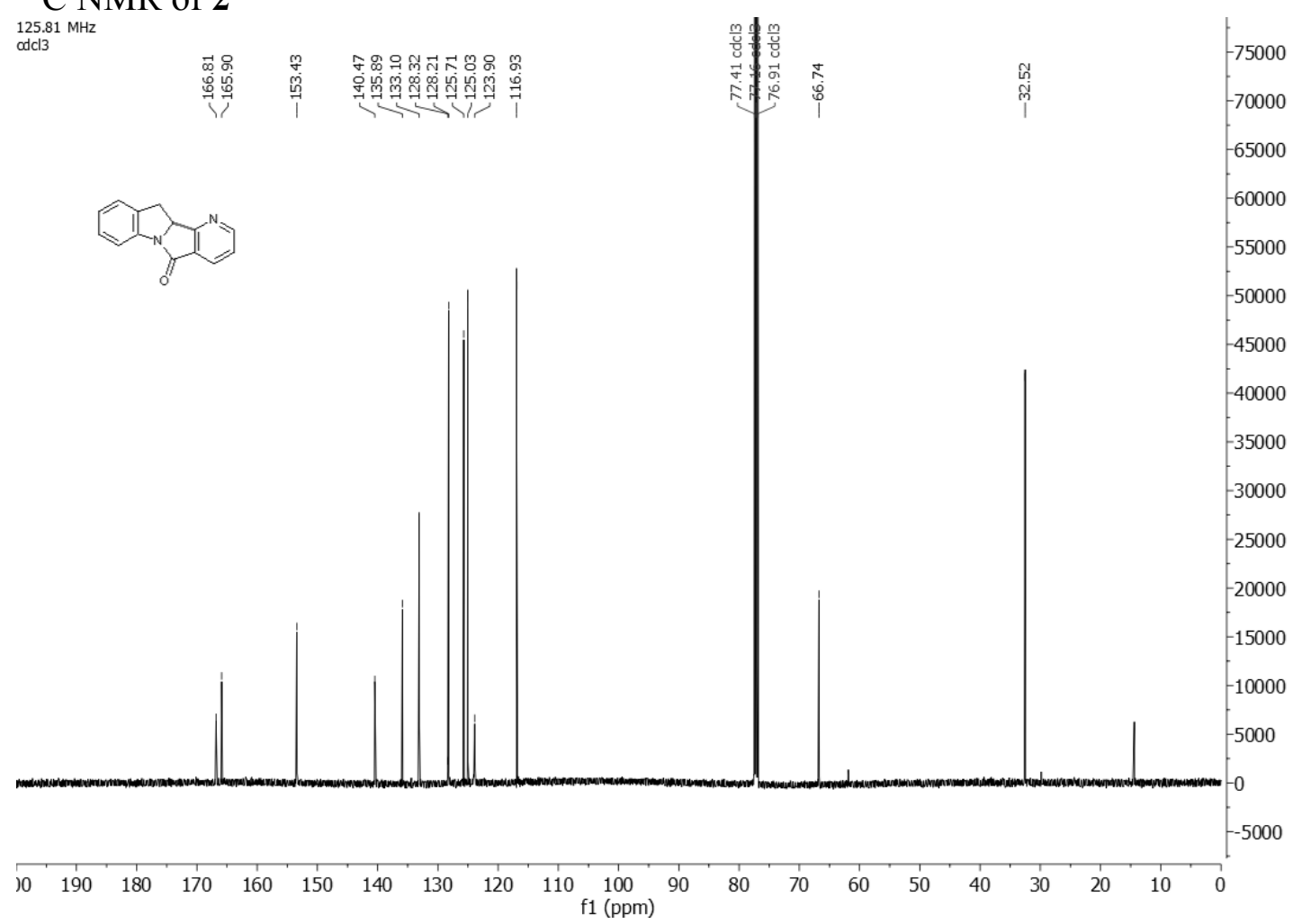


${ }^{1} \mathrm{H}$ NMR of 3

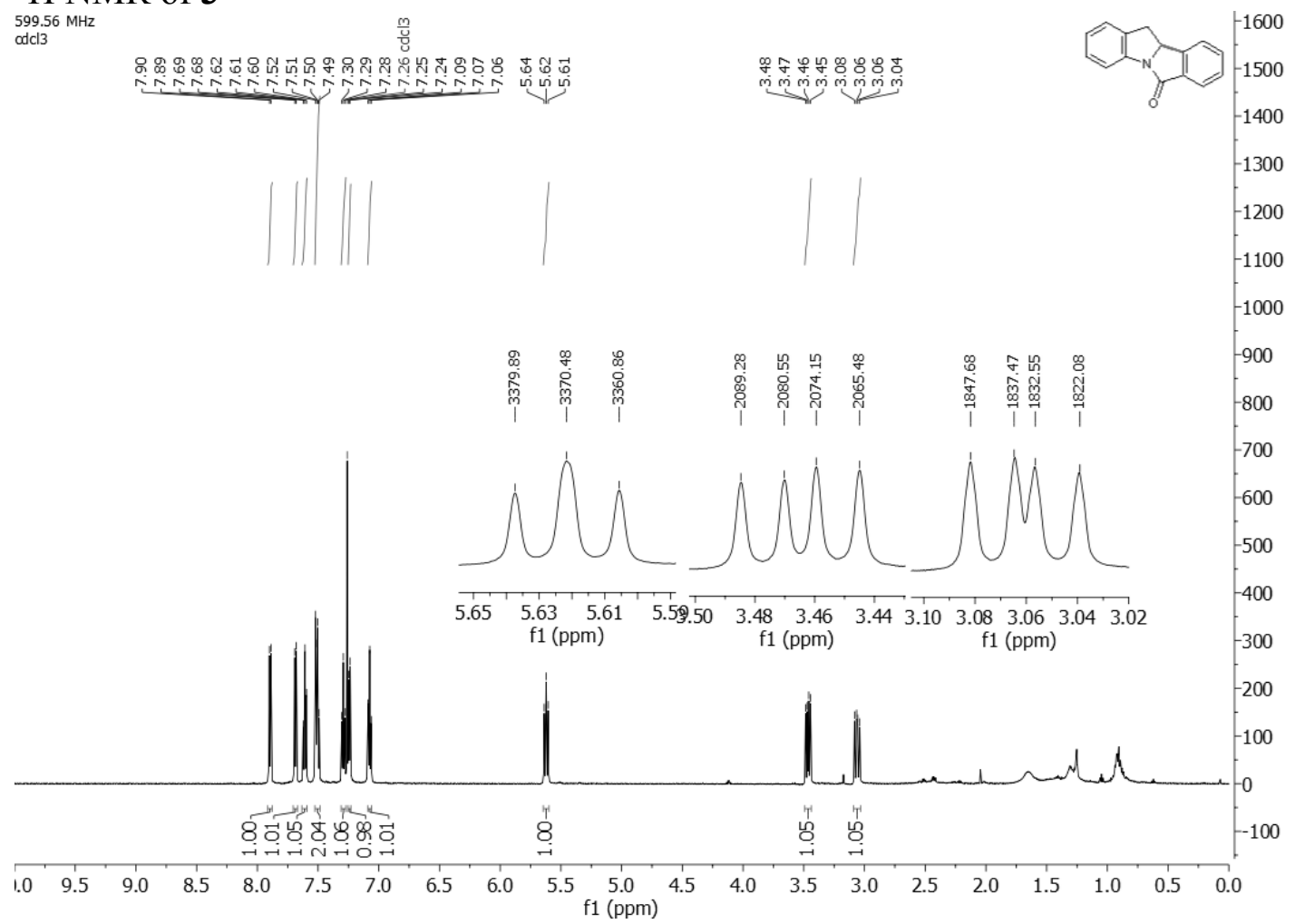

${ }^{13} \mathrm{C}$ NMR of 3

${ }_{\alpha d c 13}^{125.81} \mathrm{MHz}$

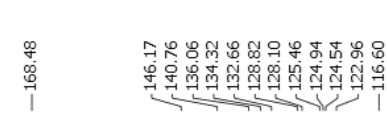

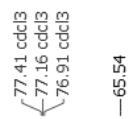

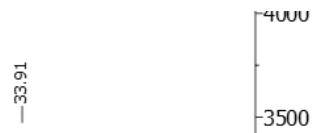

and
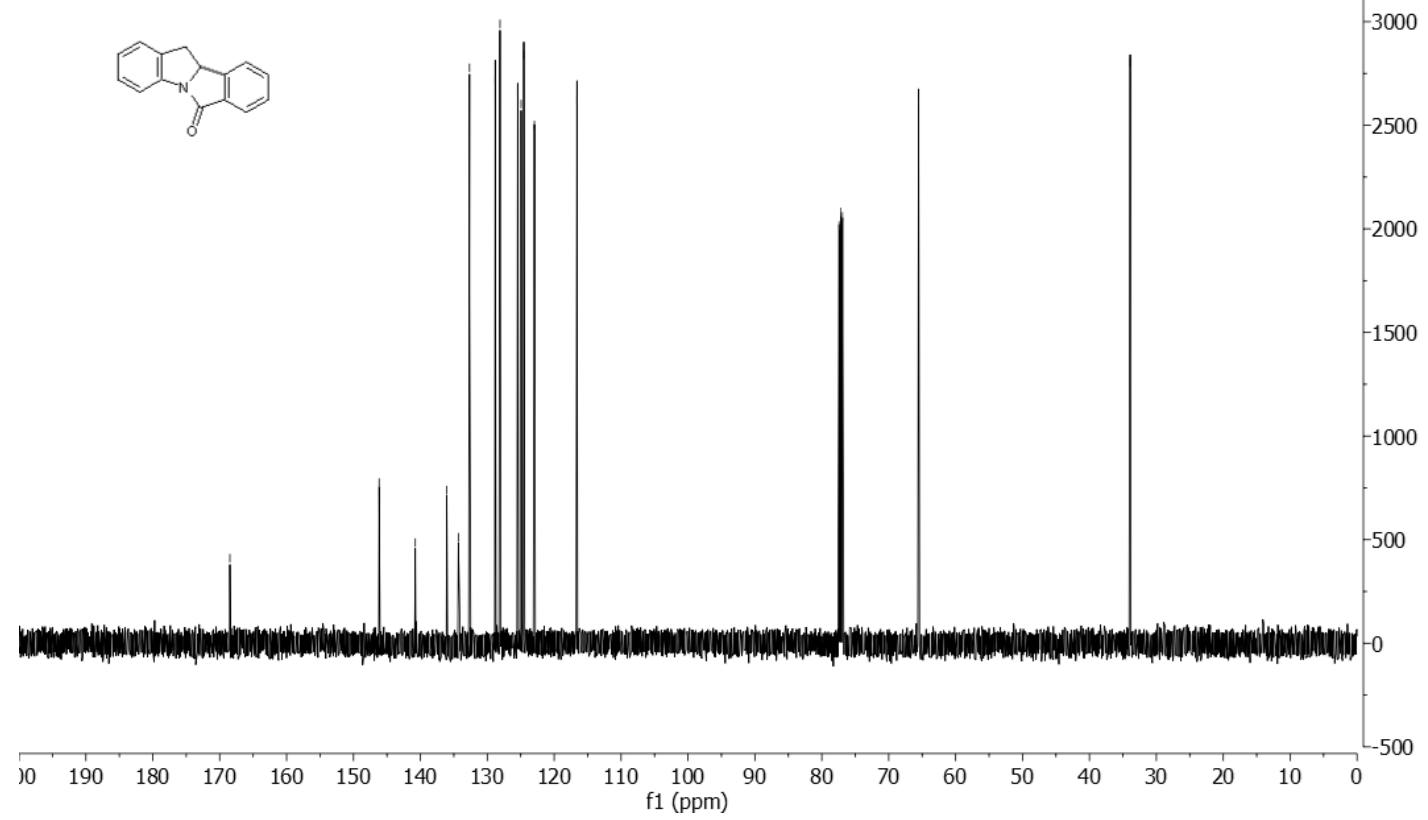
${ }^{1} \mathrm{H}$ NMR of 4

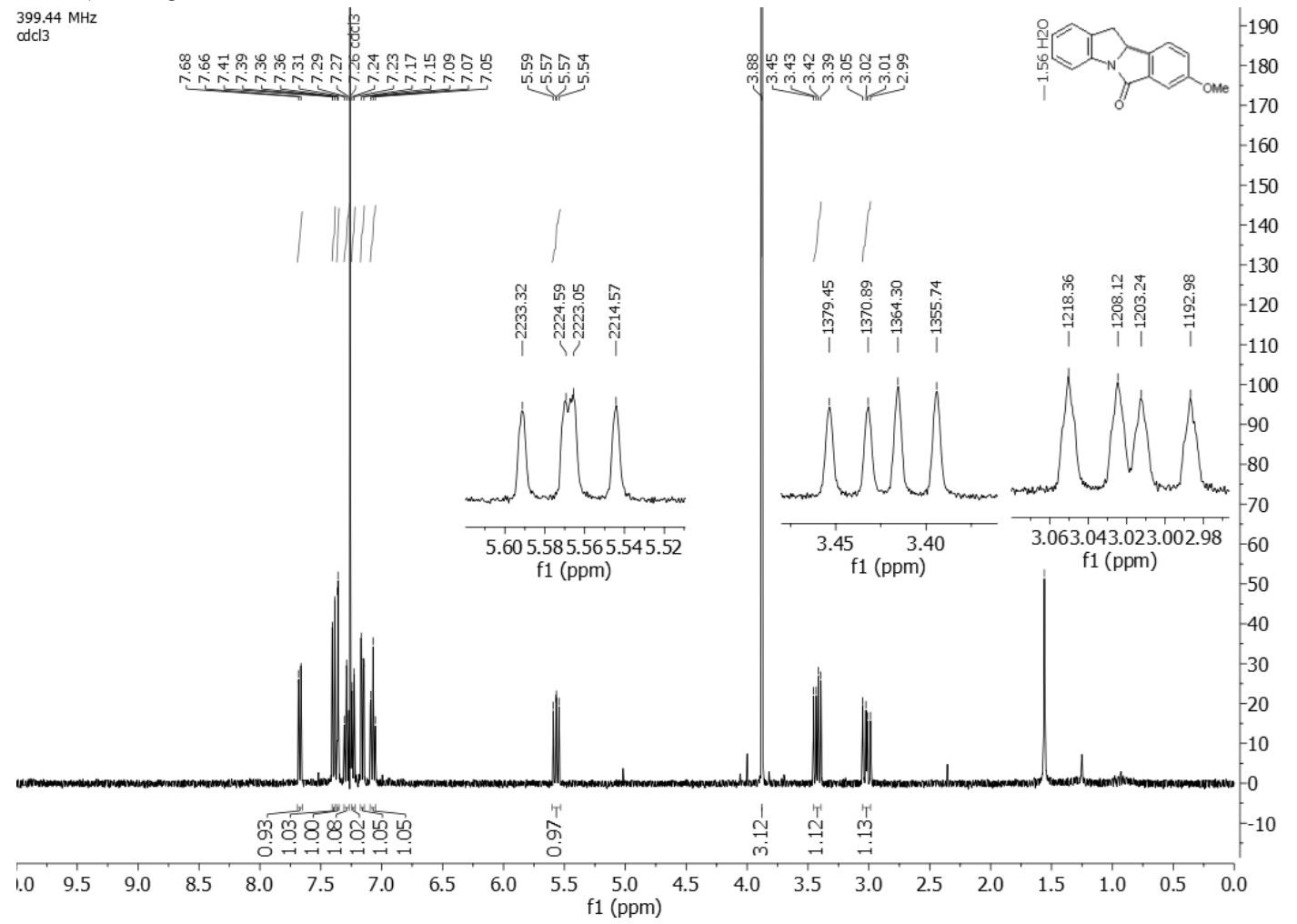

${ }^{13} \mathrm{C}$ NMR of 4

${ }_{\alpha d c 13}^{125.81} \mathrm{MHz}$

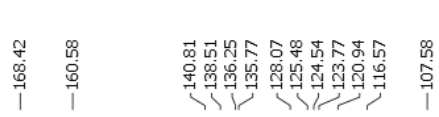

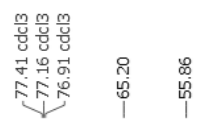

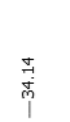

$-140$

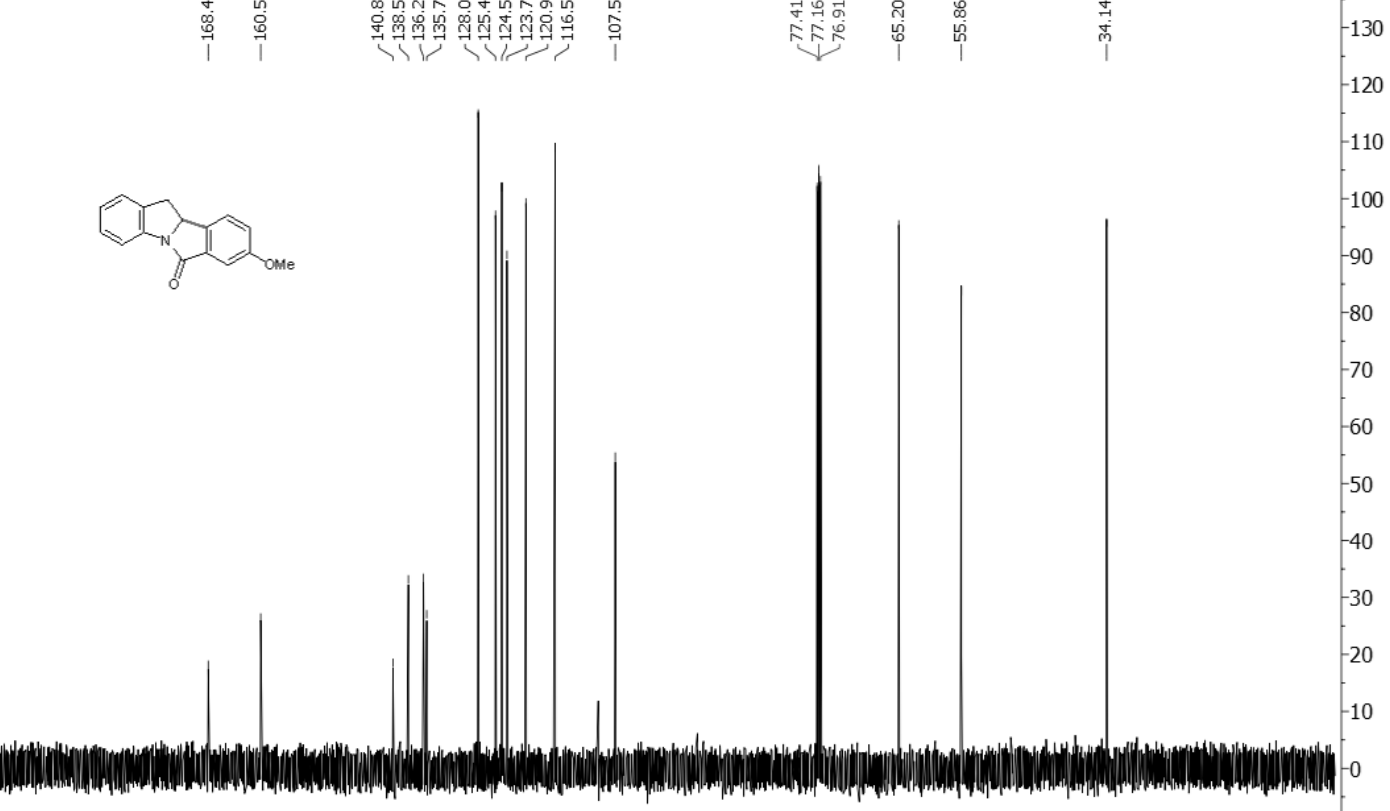

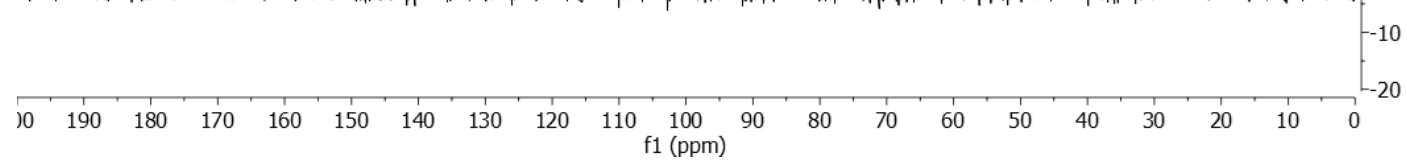




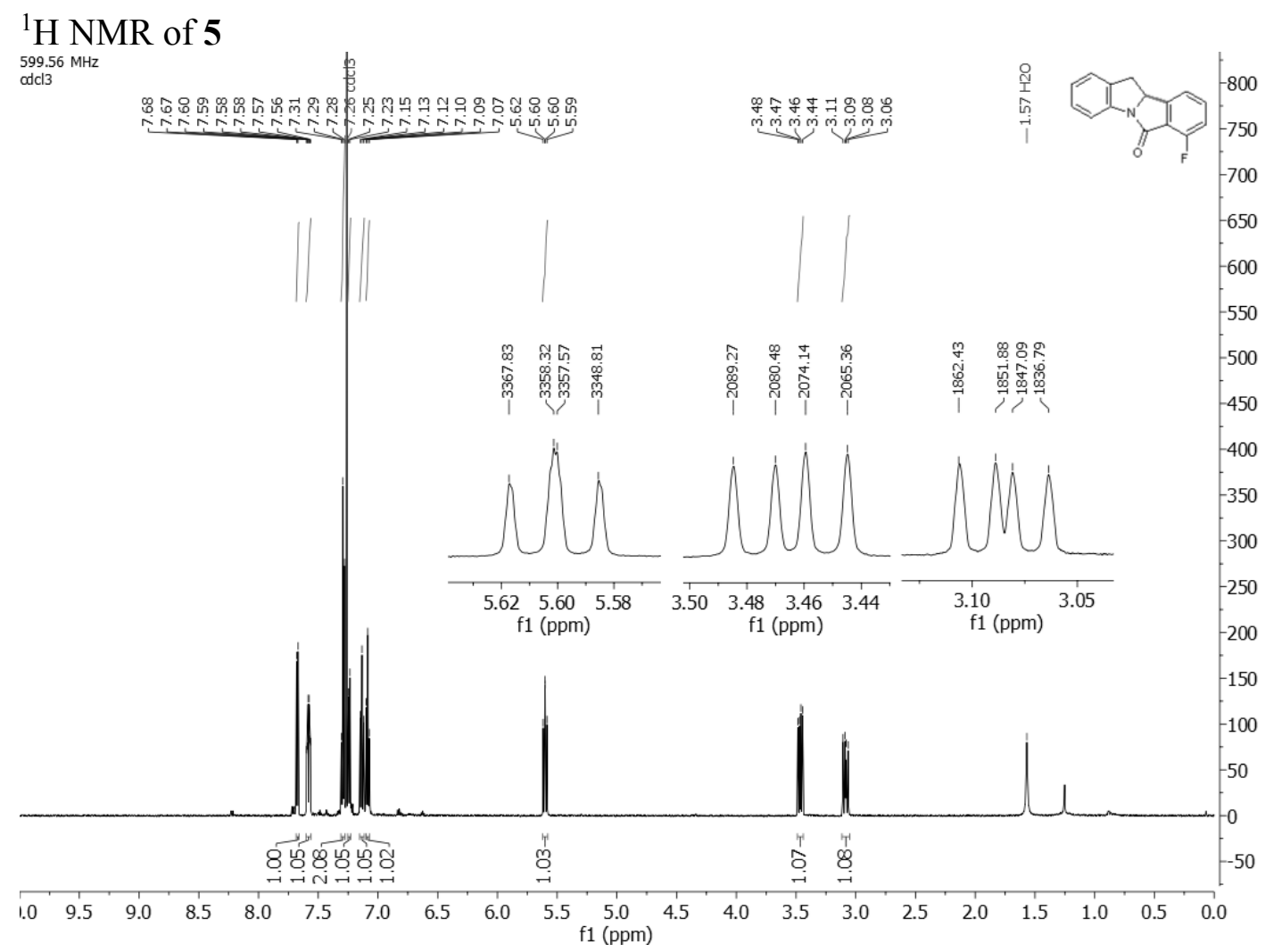

\section{${ }^{13} \mathrm{C}$ NMR of 5}

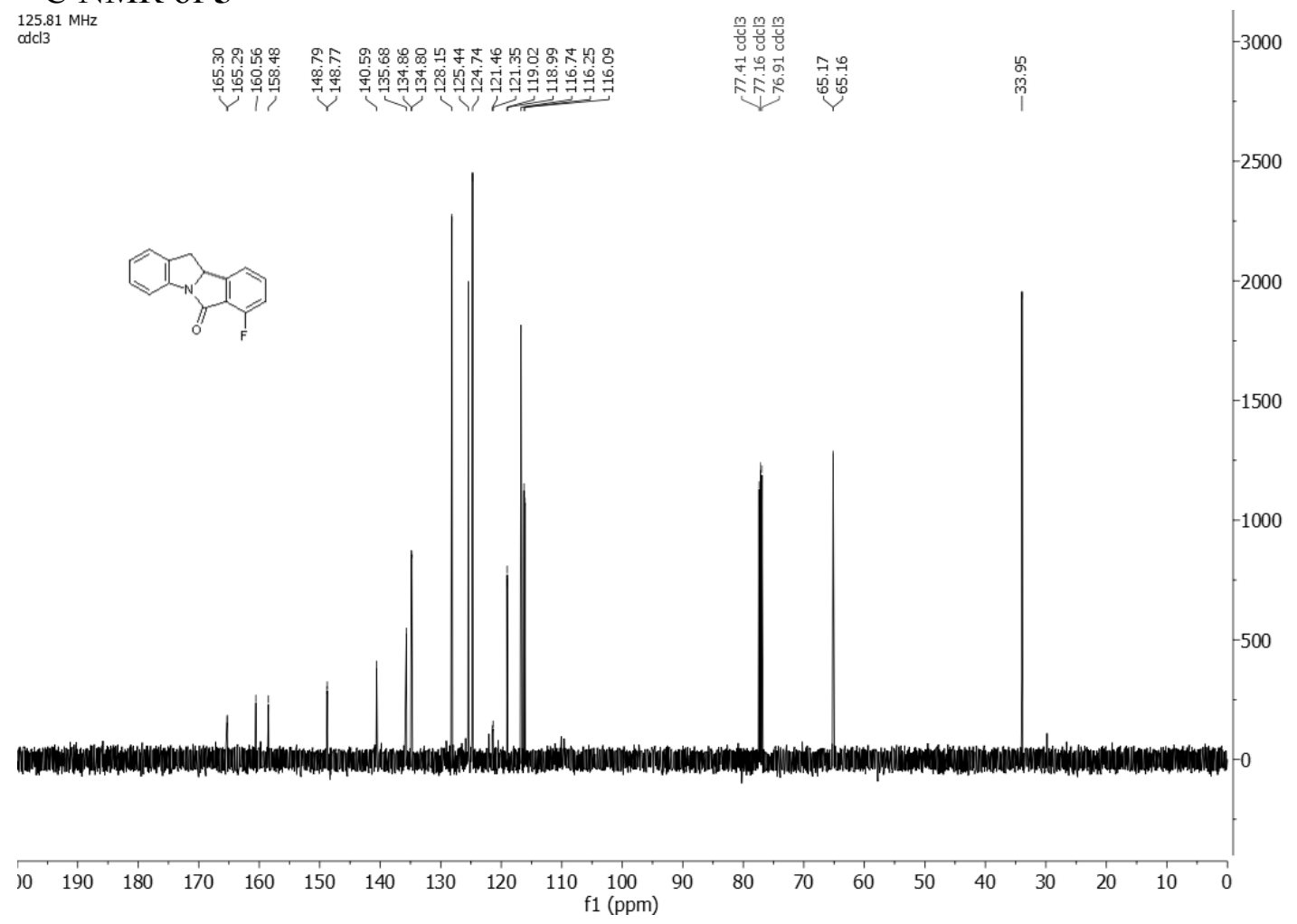




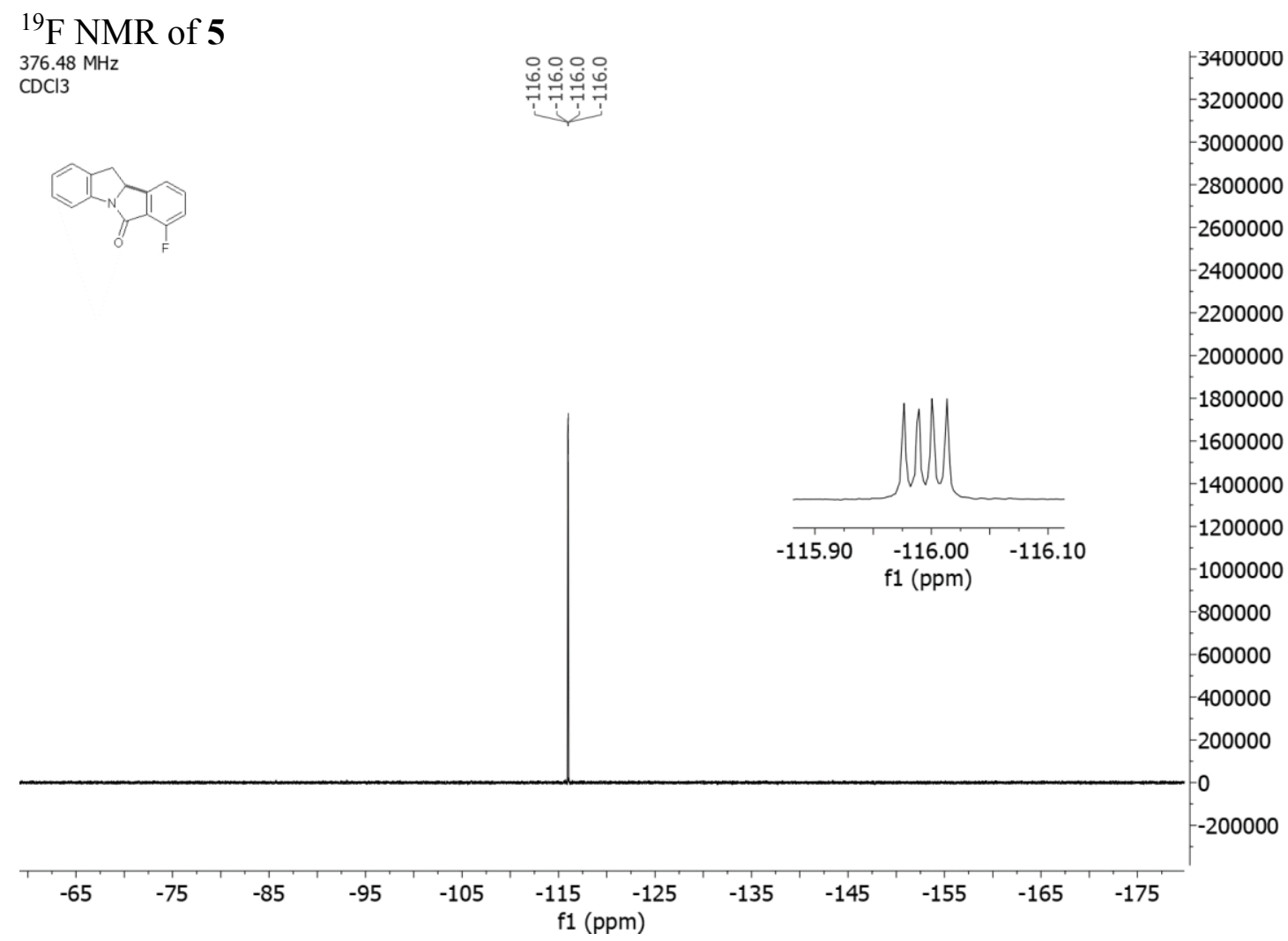


${ }^{1} \mathrm{H}$ NMR of 6

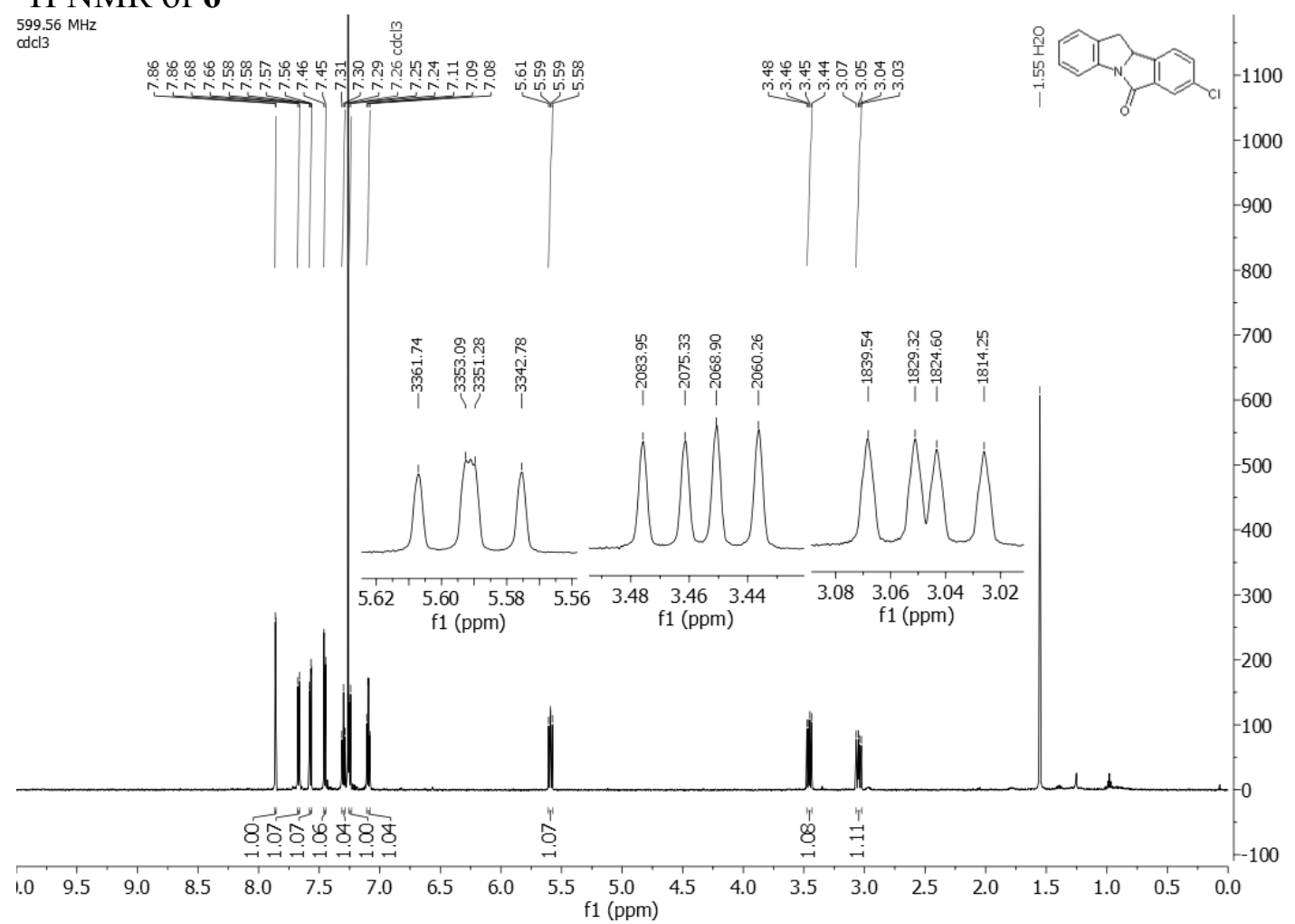

${ }^{13} \mathrm{C}$ NMR of 6

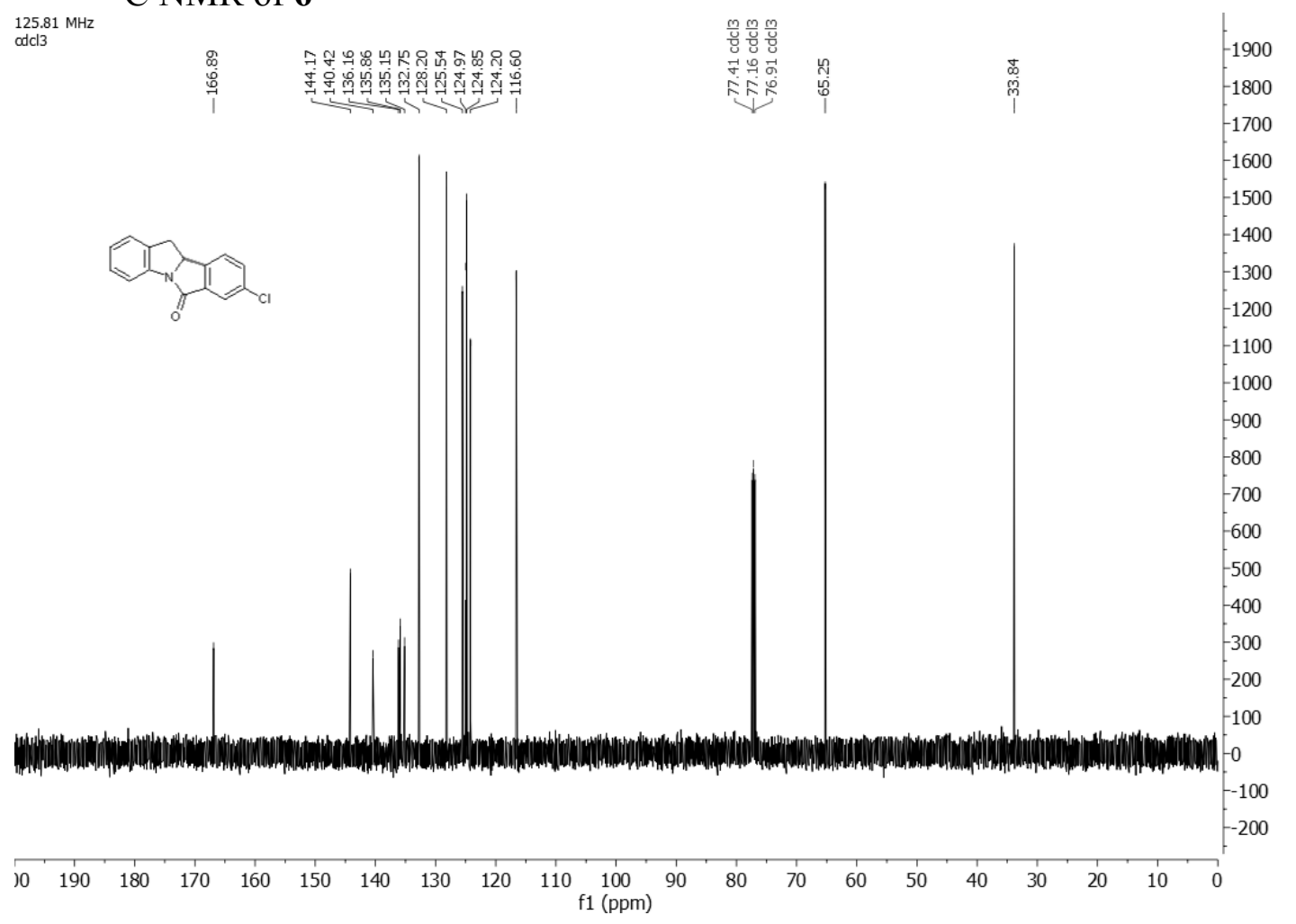


${ }^{1} \mathrm{H}$ NMR of 7

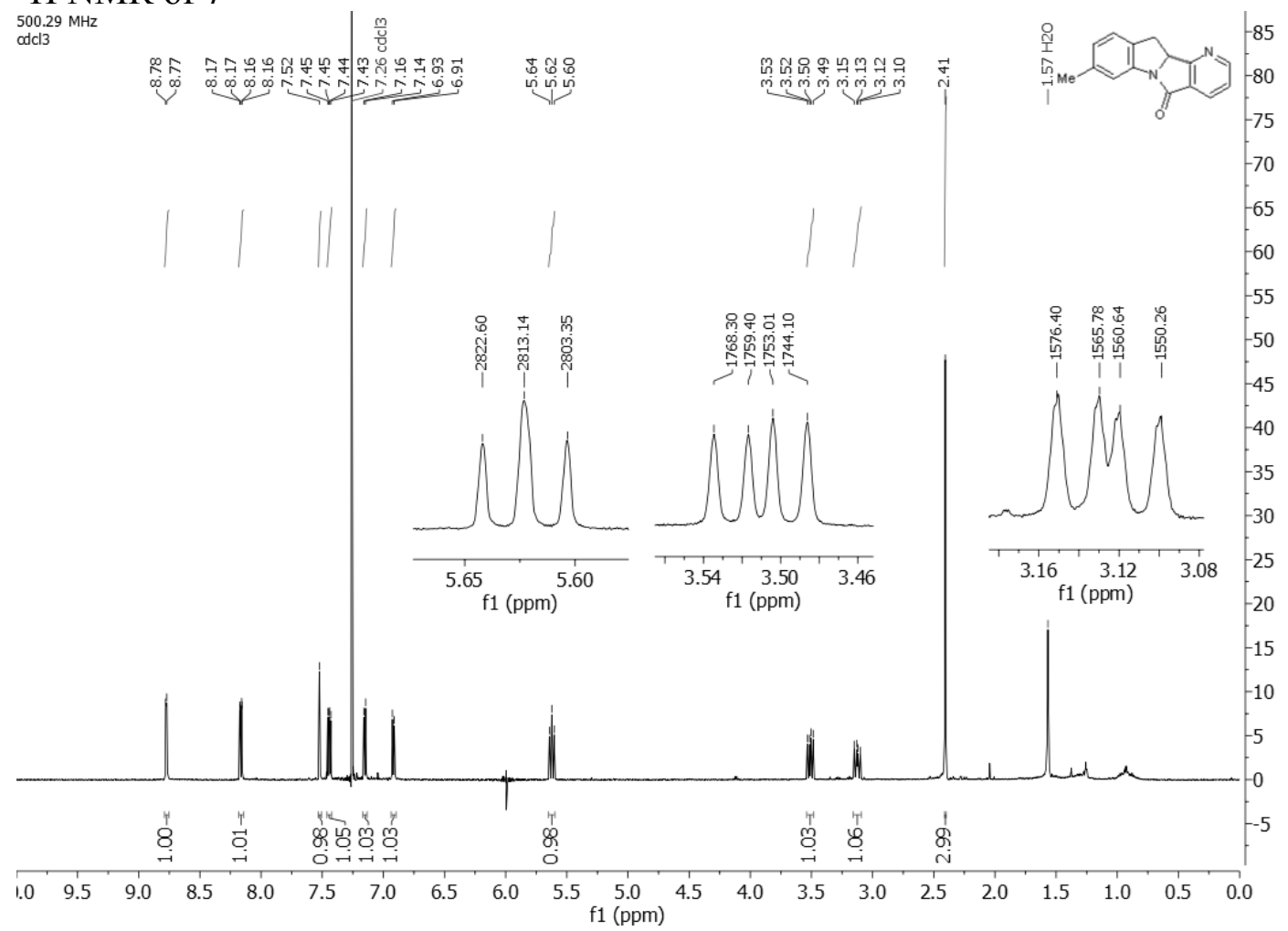

${ }^{13} \mathrm{C}$ NMR of 7

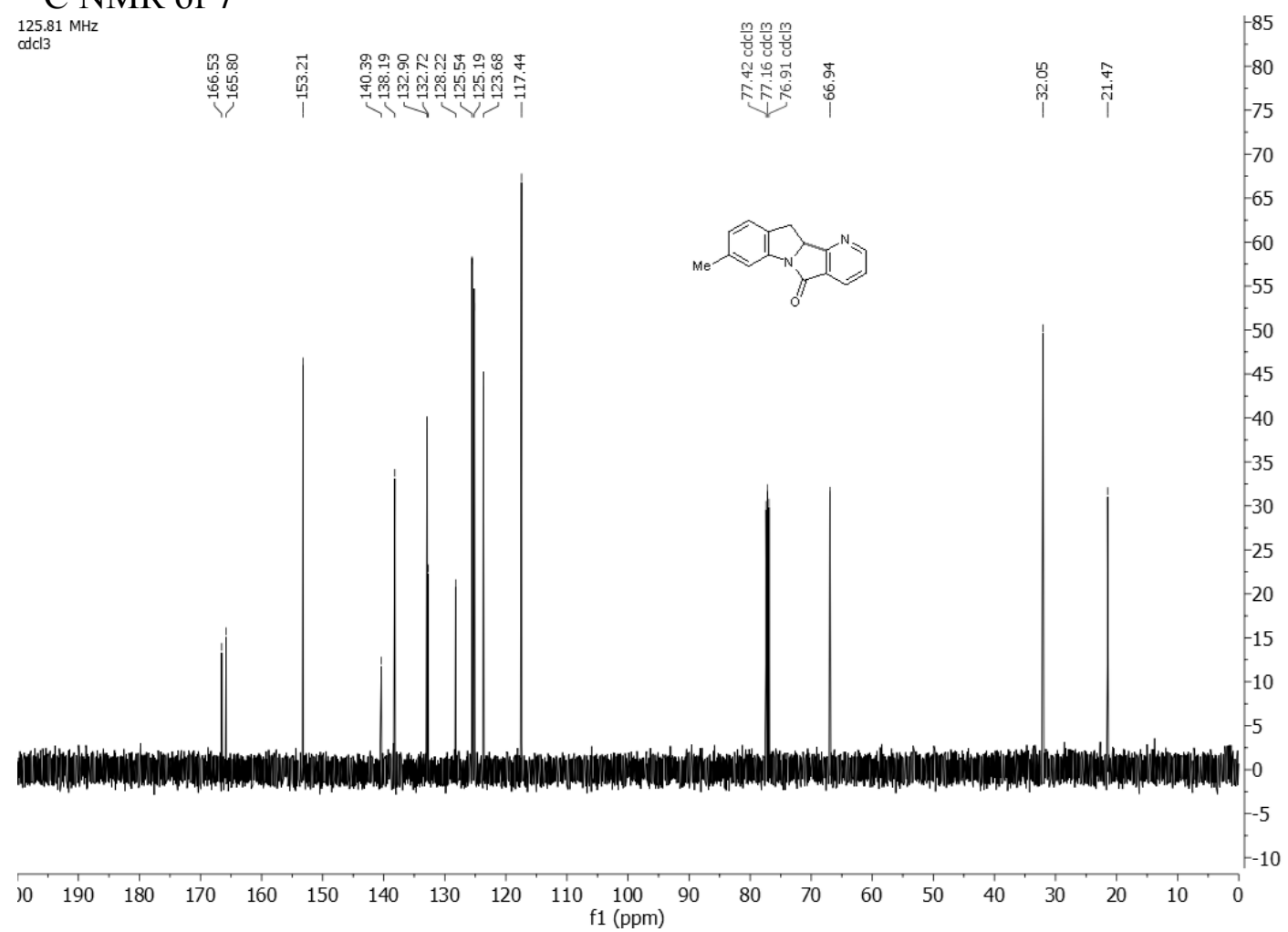




\section{${ }^{1} \mathrm{H}$ NMR of 8}

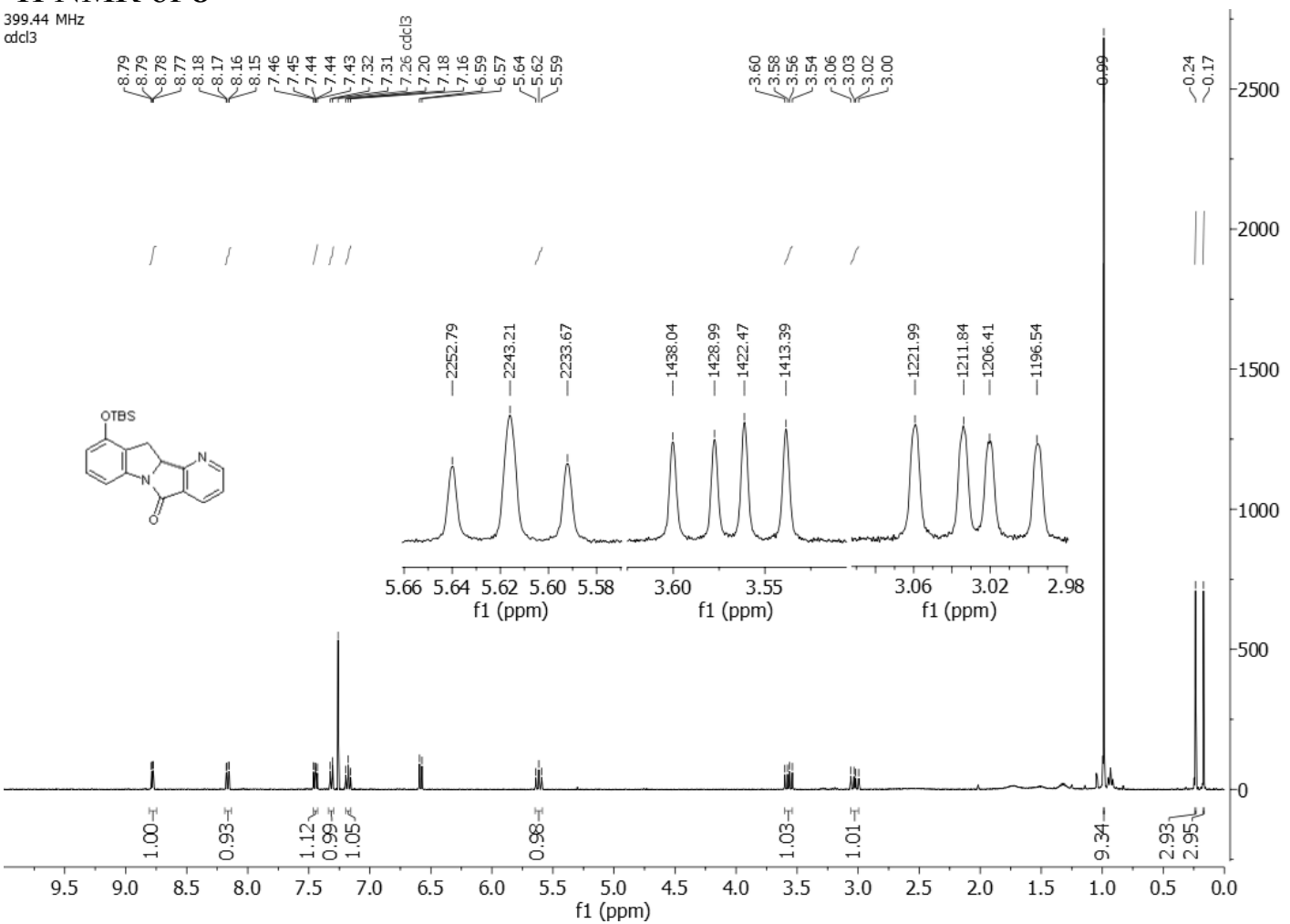

\section{${ }^{13} \mathrm{C}$ NMR of 8}

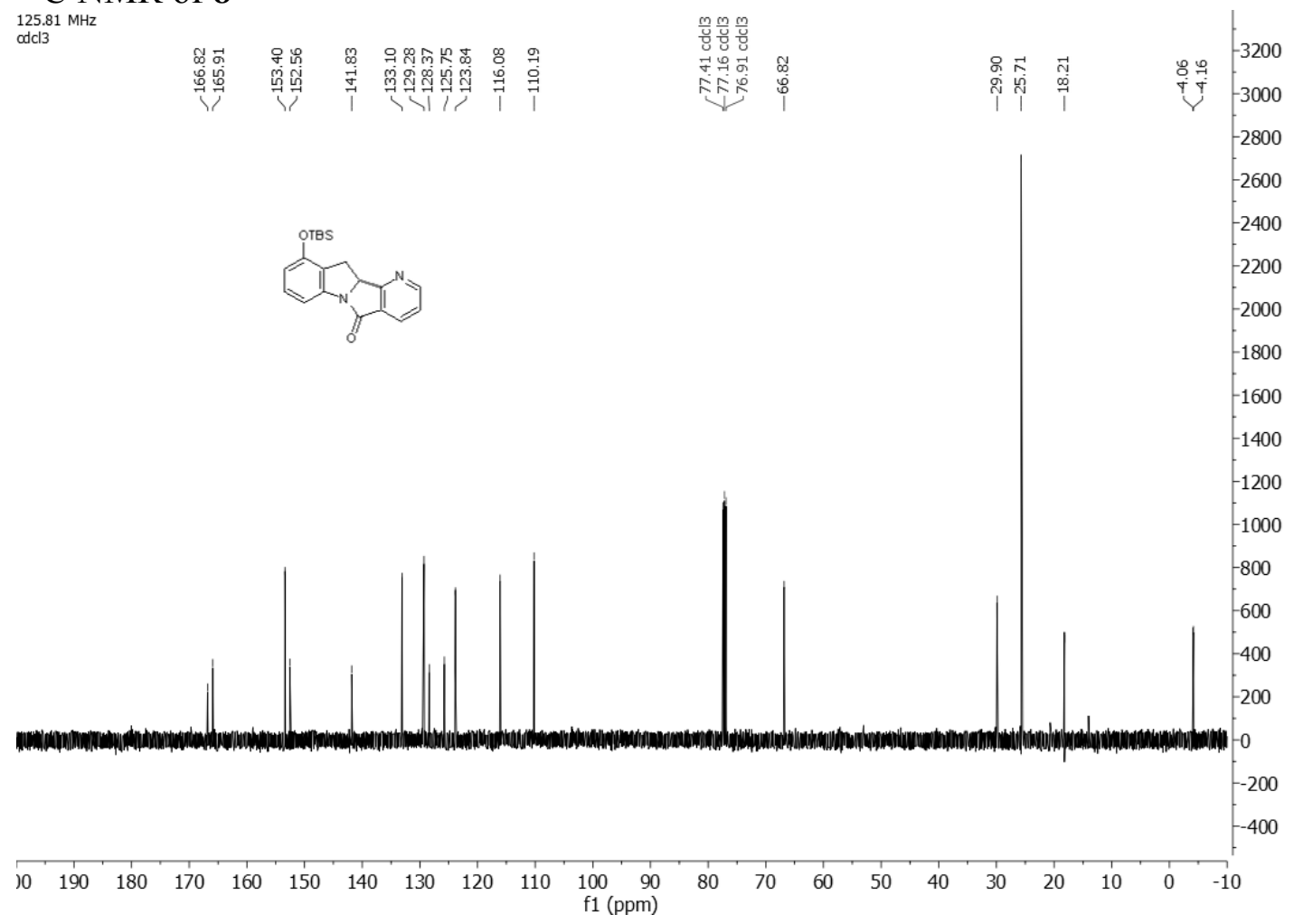




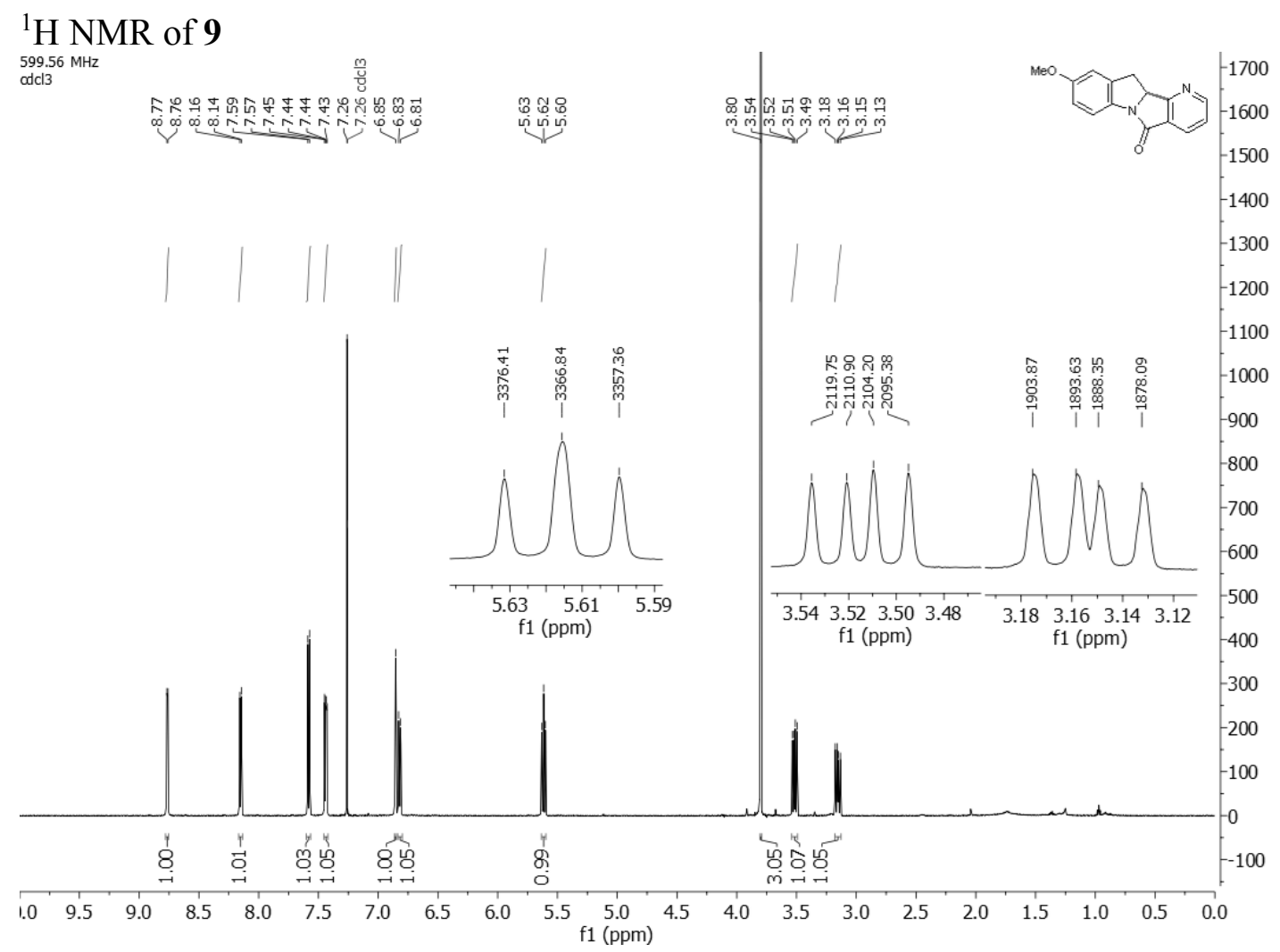

${ }^{13} \mathrm{C}$ NMR of 9

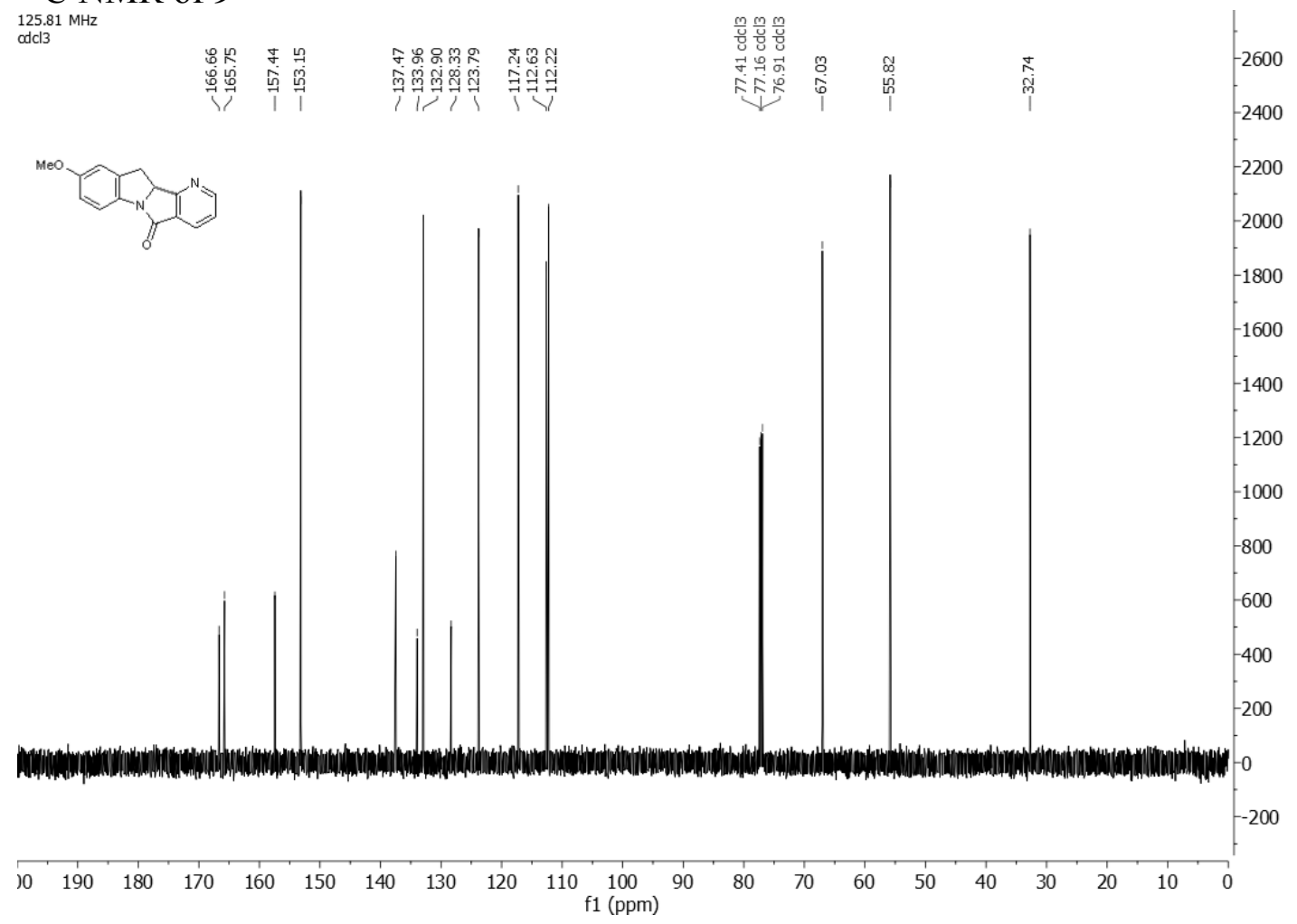


${ }^{1} \mathrm{H}$ NMR of 10

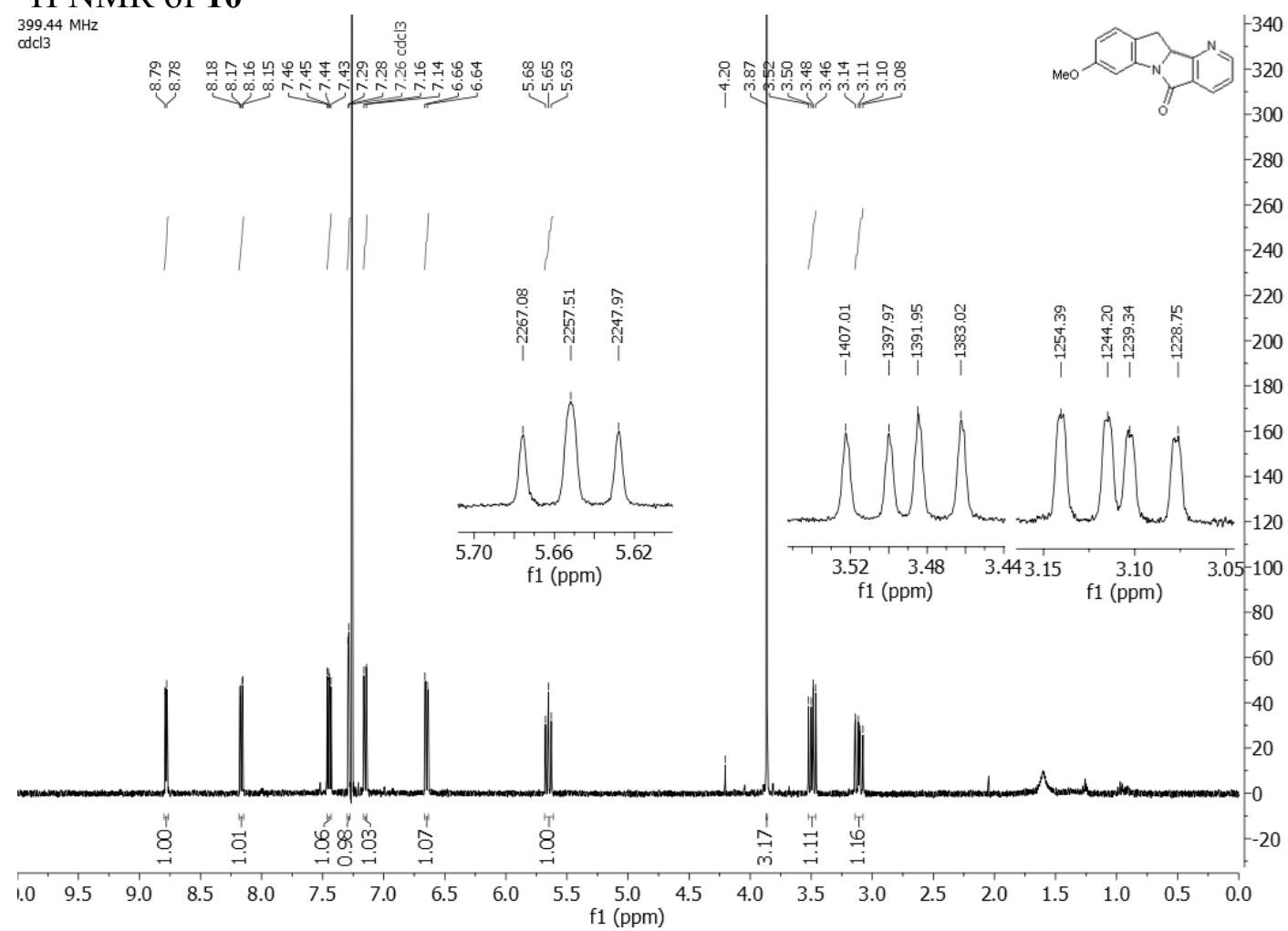

${ }^{13} \mathrm{C}$ NMR of 10

${ }_{\alpha d c 13}^{125.81} \mathrm{MHz}$
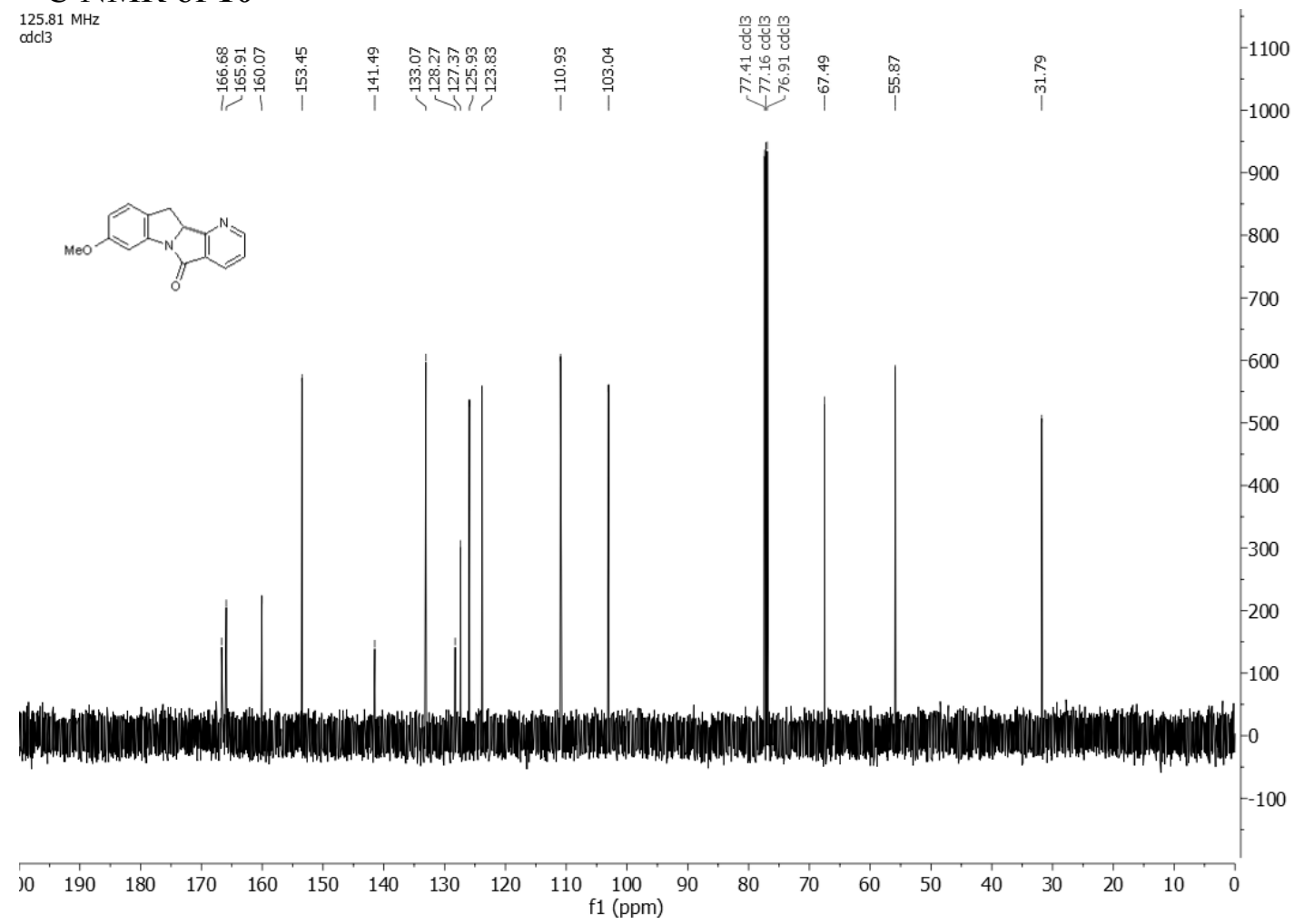
${ }^{1} \mathrm{H}$ NMR of 11

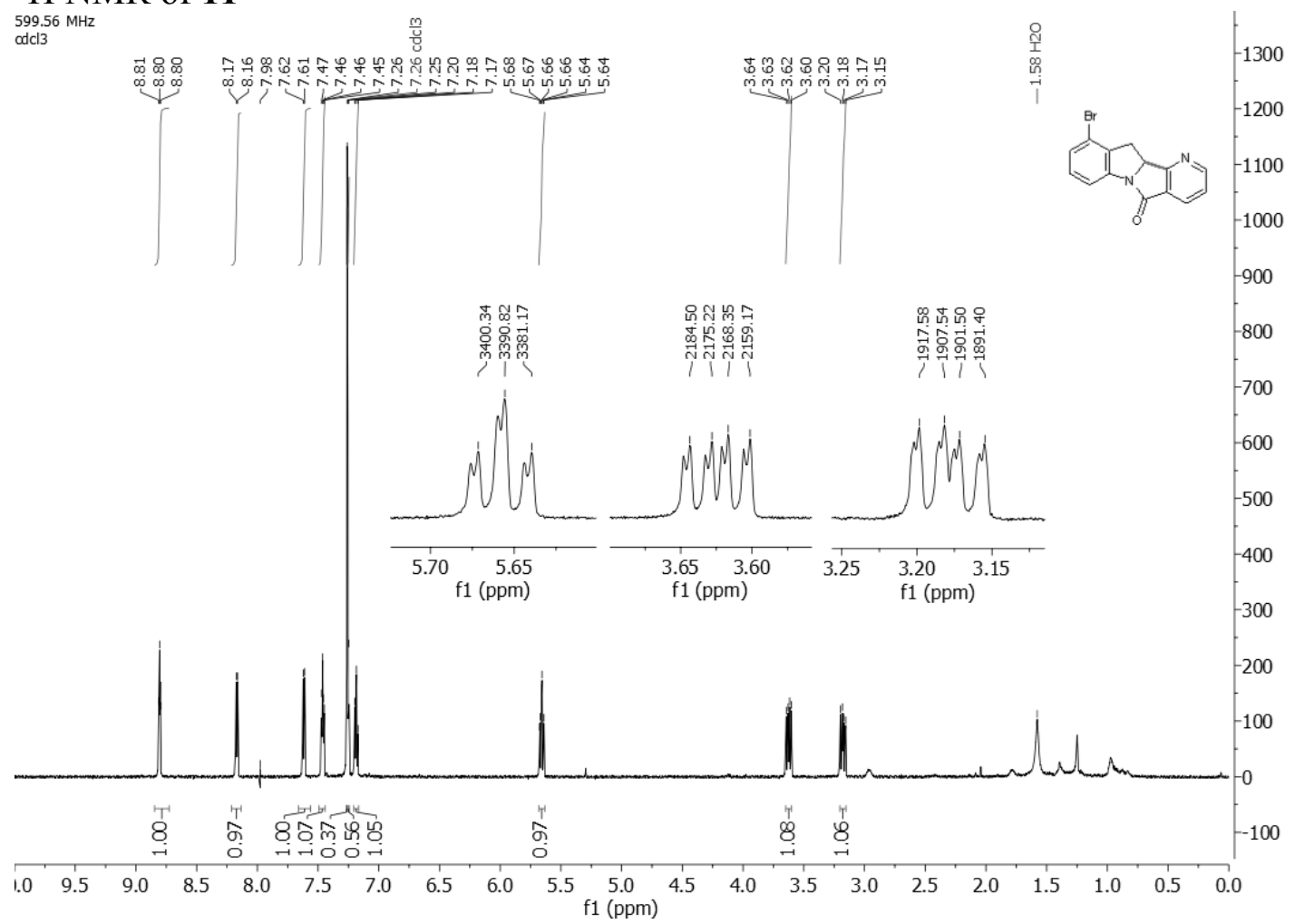

${ }^{13} \mathrm{C}$ NMR of 11 ${ }_{\alpha d c 13}^{125.81} \mathrm{MHz}$
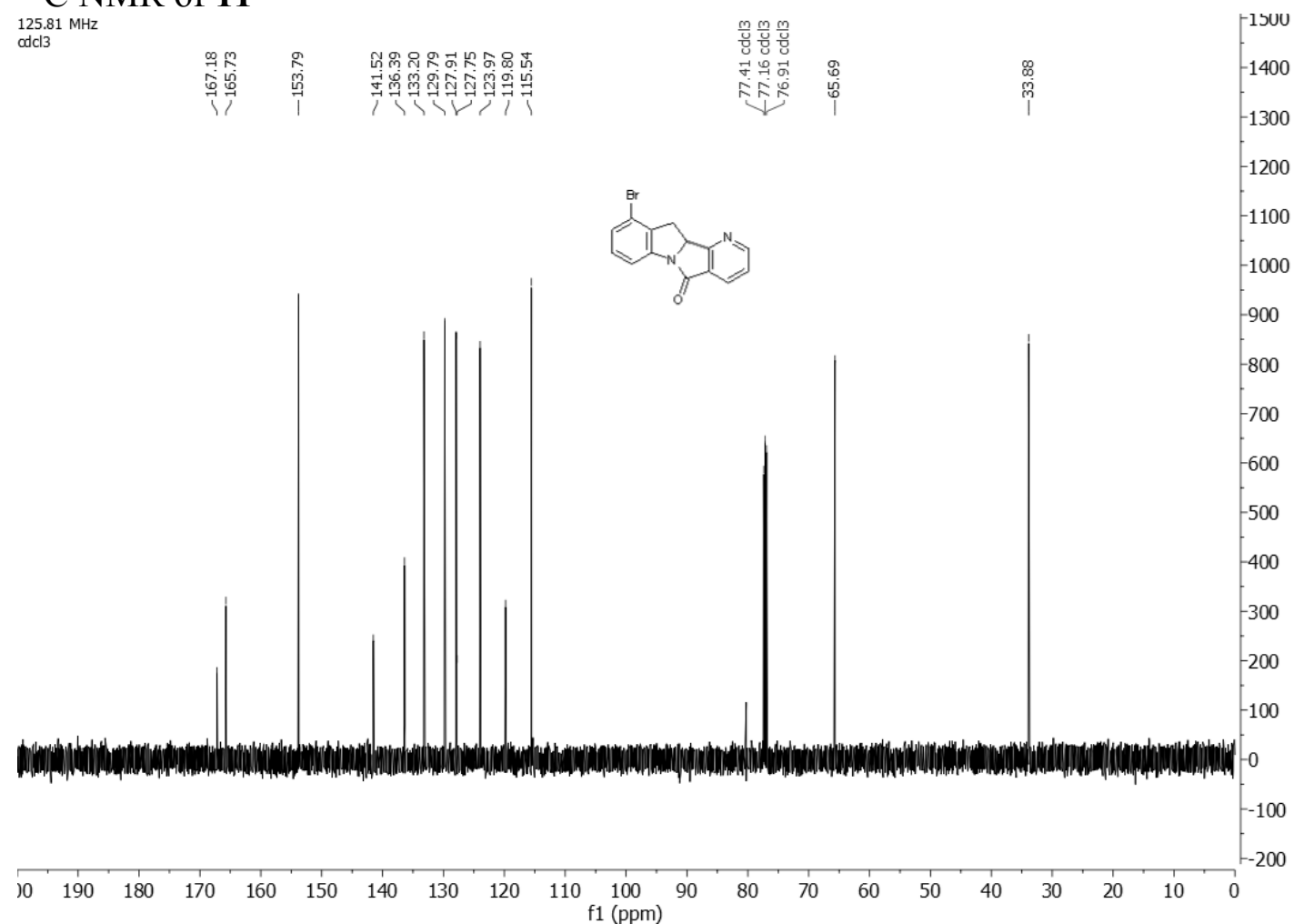
${ }^{1} \mathrm{H}$ NMR of 12

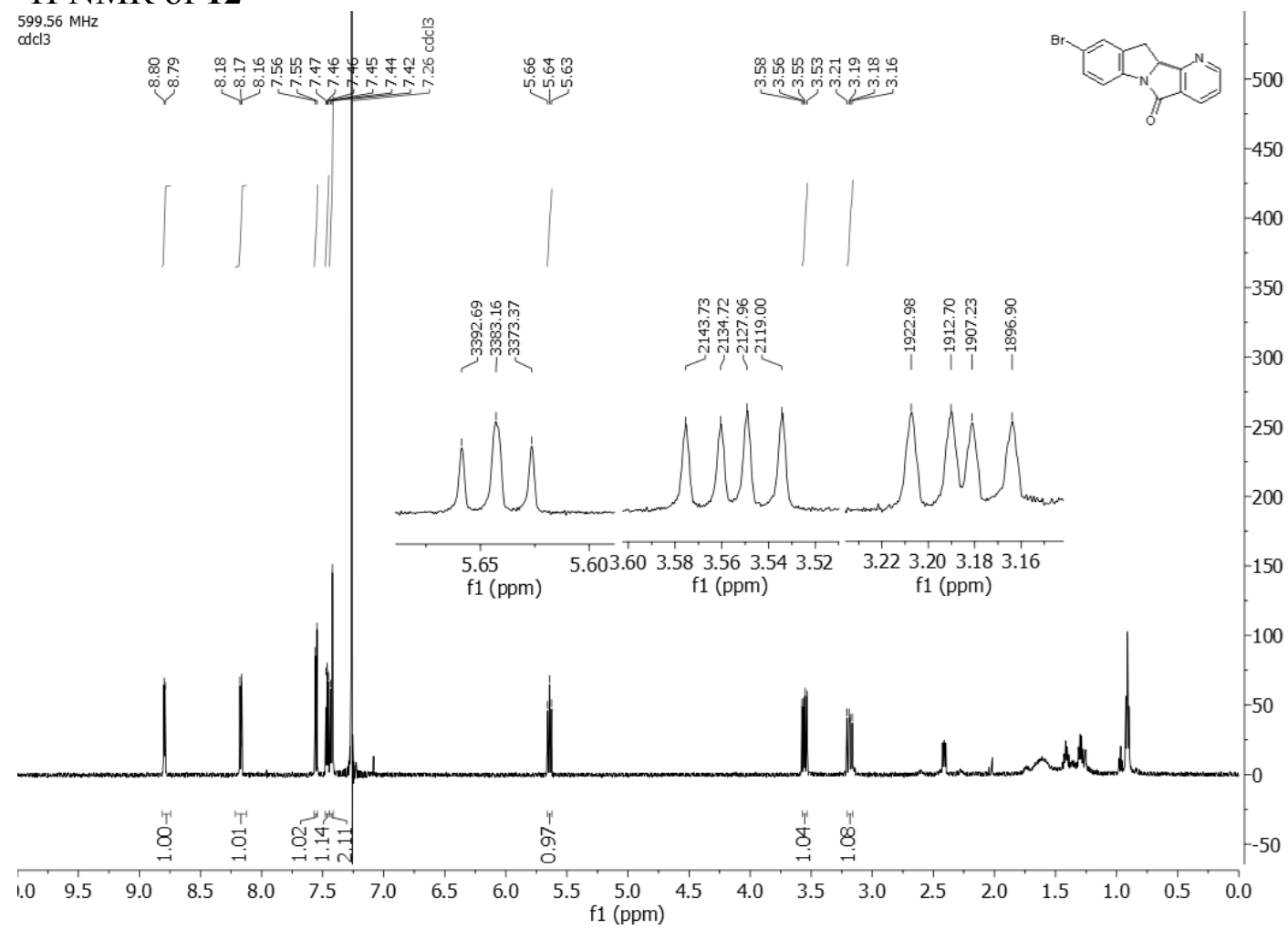

${ }^{13} \mathrm{C} \mathrm{NMR}$ of 12

${ }_{\mathrm{d} d \mathrm{cl} 3}^{1251 \mathrm{MHz}}$

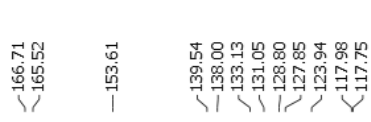

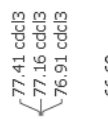

番

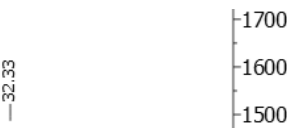

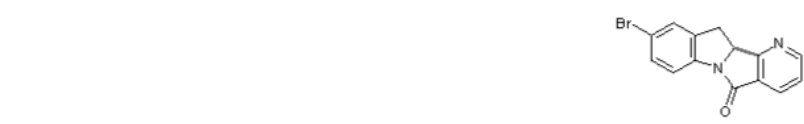

$-1300$

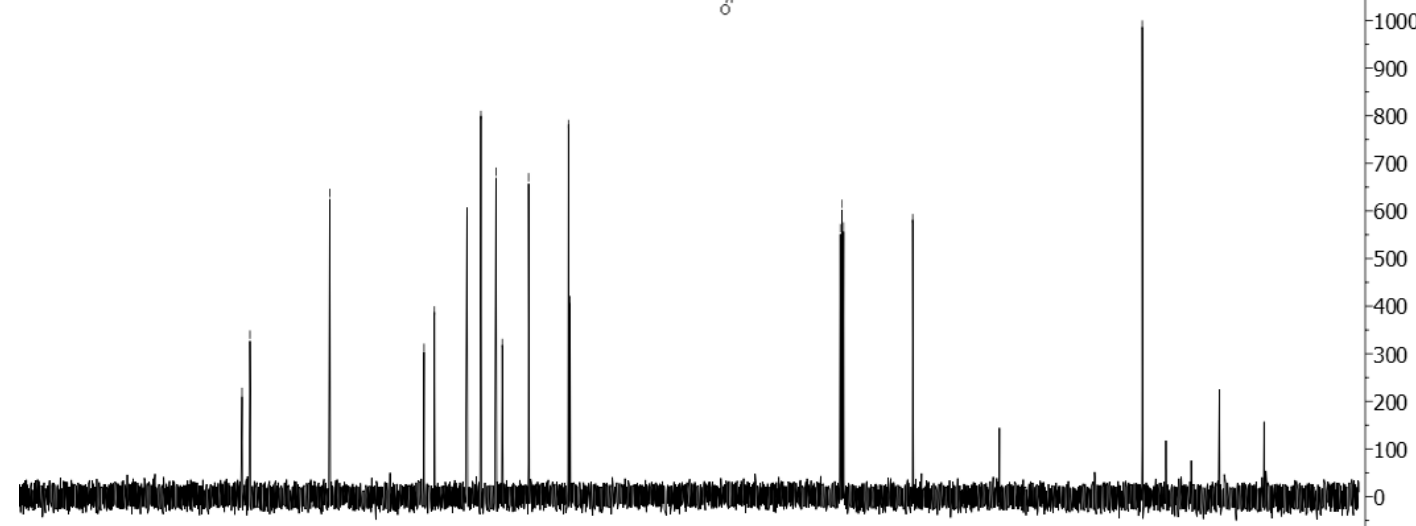

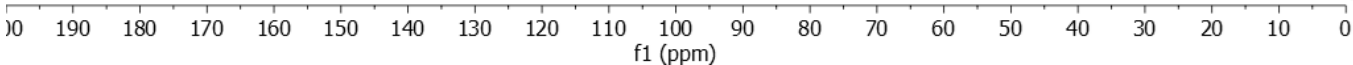




\section{${ }^{1} \mathrm{H}$ NMR of 13}

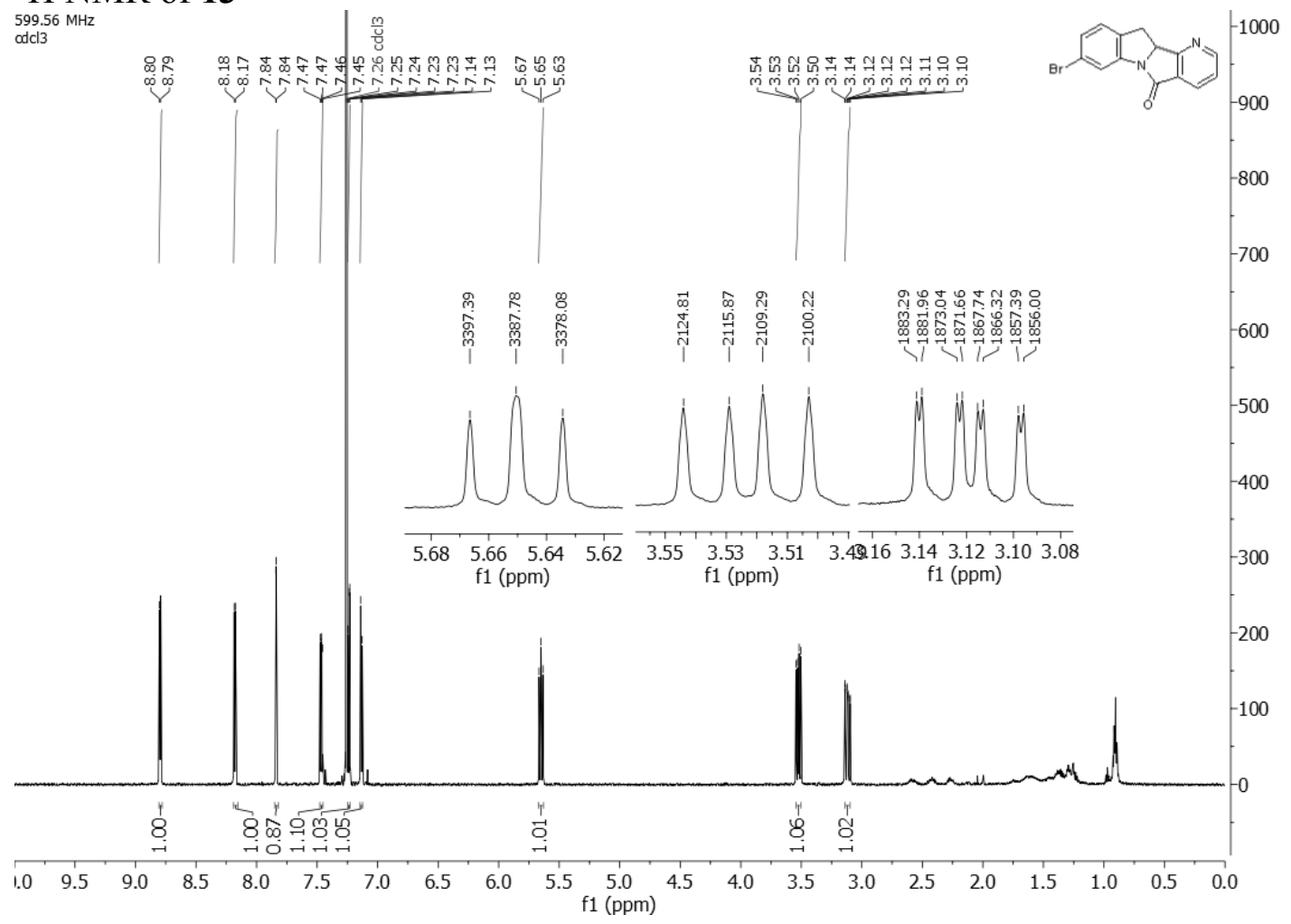

\section{${ }^{13} \mathrm{C}$ NMR of 13}

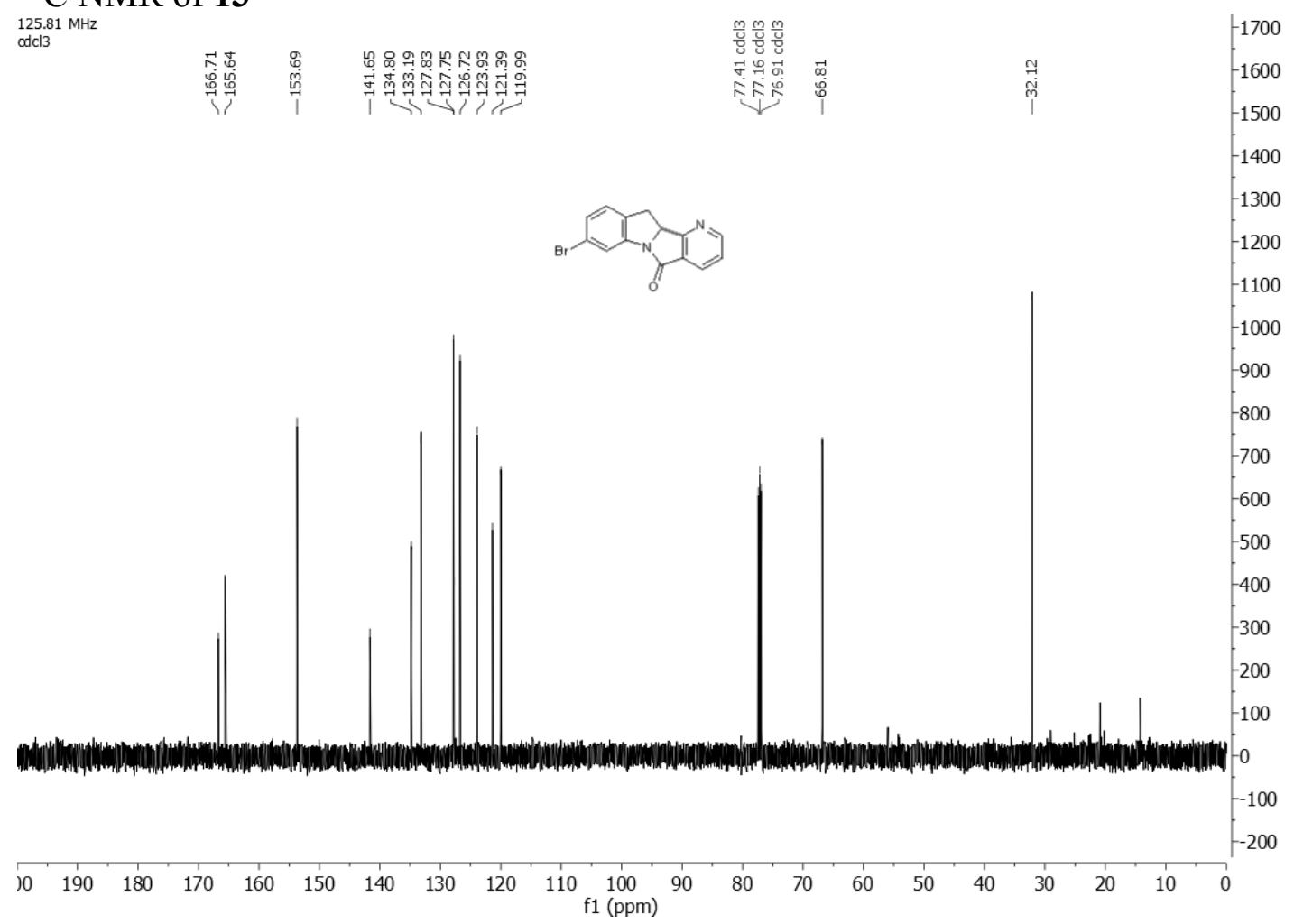

